LA-14149-T

Thesis

Approved for public release;

distribution is unlimited.

\title{
Potential Vorticity Evolution in the Co-orbital Region of Embedded Protoplanets
}

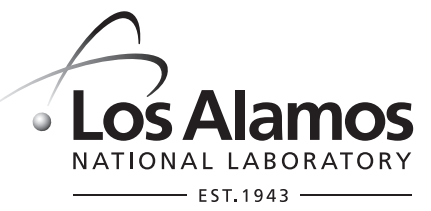

The World's Greatest Science Protecting America 
This thesis was accepted by the Department of Physics and Astronomy, Rice University, Houston, Texas, in partial fulfillment of the requirements for the degree of Doctor of Philosophy. The text and illustrations are the independent work of the author and only the front matter has been edited by the IM-1 Writing and Editing Staff to conform with Department of Energy and Los Alamos National Laboratory publication policies.

Los Alamos National Laboratory, an affirmative action/equal opportunity employer, is operated by the University of California for the United States Department of Energy under contract W-7405-ENG-36.

This report was prepared as an account of work sponsored by an agency of the United States Government. Neither the Regents of the University of California, the United States Government nor any agency thereof, nor any of their employees make any warranty, express or implied, or assume any legal liability or responsibility for the accuracy, completeness, or usefulness of any information, apparatus, product, or process disclosed, or represent that its use would not infringe privately owned rights. Reference herein to any specific commercial product, process, or service by trade name, trademark, manufacturer, or otherwise does not necessarily constitute or imply its endorsement, recommendation, or favoring by the Regents of the University of California, the United States Government, or any agency thereof. The views and opinions of authors expressed herein do not necessarily state or reflect those of the Regents of the University of California, the United States Government, or any agency thereof. Los Alamos National Laboratory strongly supports academic freedom and a researcher's right to publish; as an institution, however, the Laboratory does not endorse the viewpoint of a publication or guarantee its technical correctness. 
LA-14149-T

Thesis

Issued: September 2004

Potential Vorticity Evolution in the Co-orbital Region of Embedded Protoplanets

Josef Koller 



\section{RICE UNIVERSITY}

Vortices in the Co-orbital Region of Embedded Protoplanets

by

\section{Josef Koller}

A THESIS SUBMITTED

IN PARTIAL FULFILLMENT OF THE

REQUIREMENTS FOR THE DEGREE

\section{Doctor of Philosophy}

Approved, Thesis Committee:

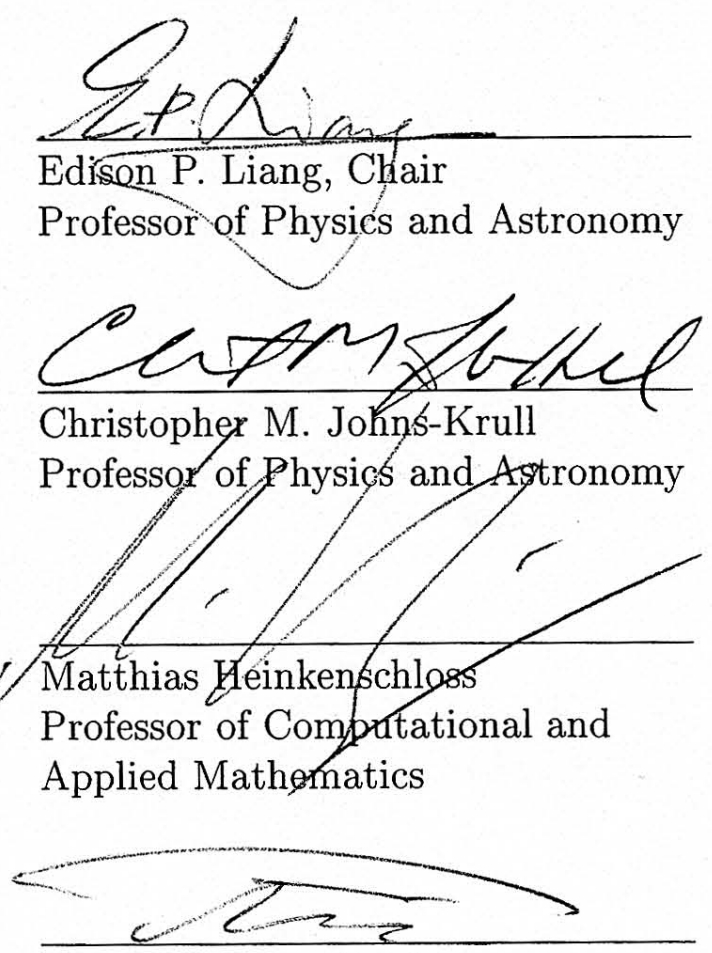

Hui Li, Adjunct Professor

Los Alamos National Laboratory

Houston, TeXas

APRIL 2004 


\section{Potential Vorticity Evolution in the Co-orbital Region of Embedded Protoplanets}

Josef Koller

Approved for public release by Los Alamos National Laboratory: LA-UR 04-2288. 
For Darcie and Madison 


\begin{abstract}
Potential Vorticity Evolution in the Co-orbital Region of Embedded Protoplanets by Josef Koller
\end{abstract}

This thesis presents two-dimensional hydrodynamic disk simulations with embedded protoplanets, emphasizing the non-linear dynamics in the co-orbital region. In particular, it demonstrates how a protoplanetary disk responds to embedded low mass planets at the inviscid limit. Since the potential vorticity (PV) flow is not conserved, due to the spiral shocks and possibly boundary layer effects emanating from the planet, the PV profile develops inflection points which eventually render the flow unstable. Vortices are produced in association with the potential vorticity minima. Born in the separatrix region, these vortices experience close encounters with the planet, consequently exerting strong torques on the planet. The existence of these vortices, if confirmed, have important implications on planetary migration rates. The formation of vortices is discussed in more detail and a key parameter is found which depends solely on planet mass and sound speed. With this key parameter, one can predict the disk evolution, PV growth rates, and threshold conditions for forming vortices in the co-orbital region. An analytical estimate for the change of PV due to shocks is compared to the actual change in PV in the hydrodynamic simulations. They match well except in the inner region where vortices form. In addition, extensive resolution tests were carried out but uncertainties remain about the physics of this particular region. 


\section{Acknowledgements}

I would like to extend a special thank you to the following people: to Hui Li, my mentor in Los Alamos who has taught me many things on hydrodynamics and accretion disk physics and for his simulation code on which this research is based on; to Edison Liang, my advisor at Rice University, who introduced me to the internship at Los Alamos National Laboratory; to Burton Wendroff, the inventor of the Lax-Wendroff scheme, who patiently explained numerical hydrodynamics to me and invited me for many mountain biking trips; to all my friends in Houston and Europe, who endured my personality and shared all the happy and not so happy moments with me; and finallly to my family including my loving wife Darcie, my daughter Madison, and my family in Austria, who, throughout this project, have shown more support, love, and patience than I could ever have hoped for.

This research was performed under the auspices of the Department of Energy. It was supported by the Laboratory Directed Research and Development Program at Los Alamos and by the Institute of Geophysics and Planetary Physics IGPP/1109 and IGPP/1326, in Los Alamos as well. I was also supported in part by NASA through NAG5-9223. 


\section{Contents}

1 Introduction and Motivation 1

2 Model Description $\quad 7$

2.1 Numerical Scheme ................... 7

2.2 Hydrodynamic Equations . . . . . . . . . . . . . . 10

2.3 Angular Momentum Conservation in Rotating Coordinates . . . . . 13

2.4 Equation of State . . . . . . . . . . . 15

2.5 Initial and Boundary Conditions . . . . . . . . . . . 17

3 Smoothing Factor $\quad 20$

3.1 Effects of the Smoothing Factor . . . . . . . . . . 20

3.2 Three-Dimensional Approximation ............. 25

4 Density Evolution and Gap Formation 32

5 Torque Evolution $\quad 42$

5.1 Torque Equations . . . . . . . . . . . . . . 42 
5.2 Phase Transition . . . . . . . . . . . . . . . . . 44

5.3 Phase III Transition . . . . . . . . . . . . . . 45

$6 \quad$ PV, Shocks, and Instabilities $\quad 51$

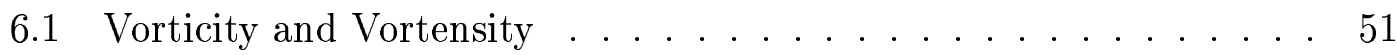

6.2 PV Evolution and Secondary Instabilities . . . . . . . . . 55

$\begin{array}{lll}7 & \text { Parameter Studies } & 65\end{array}$

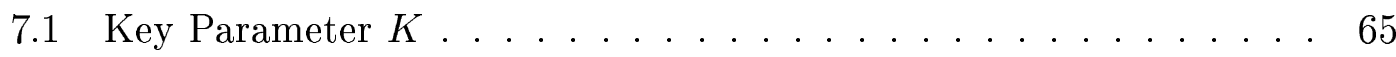

7.2 Torque Evolution and Sound Speed Dependence . . . . . . . . . 67

7.3 Growth Rate Parameter . . . . . . . . . . . . 73

7.4 Functional Dependence on Planet Mass . . . . . . . . . . . 73

7.5 Functional Dependence on Sound Speed . . . . . . . . . . 77

8 Particle Paths $\quad 89$

9 Shock Structure and PV Jumps 100

9.1 Shock Structure . . . . . . . . . . . . 100

10 Some Multi Planet Simulations $\quad 114$

11 Code Evaluation and Comparison $\quad 122$

11.1 Steady State Disk Test . . . . . . . . . . . . 122

11.2 Resolution Tests . . . . . . . . . . . . . . 123

11.3 Comparison with VH1 .................. 126 
12.1 Initial setup and orbital properties . . . . . . . . . . 132

12.2 Results from the Three-Body Simulations . . . . . . . . . . . 141

12.3 Particle Response with Increasing Planet Mass . . . . . . . . . . . . 142

12.4 Analytic Approach to Smoothing Factor . . . . . . . . . . . 145

13 Results and Discussion 


\section{List of Figures}

2.1 Sound speed temperature relation at $1 \mathrm{AU} \ldots \ldots \ldots \ldots$

3.1 Potential of planet with smoothing factor $\ldots \ldots \ldots \ldots . \ldots 22$

3.2 Density profile for different smoothing lengths $\ldots \ldots \ldots \ldots 23$

3.3 Vorticity depending on smoothing factor . . . . . . . . 24

3.4 Torque depending on smoothing factor $\ldots \ldots \ldots \ldots$

3.5 Correction for three-dimensional approximation $\ldots \ldots \ldots \ldots$

3.6 Original radial force vs. three-dimensional version . . . . . . . 30

3.7 Standard planetary force over the three-dimensional force . . . . . . 31

4.1 Snapshot of the disk surface density at $t=50 P \ldots \ldots 35$

4.2 Snapshot of the disk surface density at $t=200 P \ldots \ldots 36$

4.3 Snapshot of the disk surface density at $t=500 P \ldots \ldots 37$

4.4 Density profile for different sound speeds $\ldots \ldots \ldots \ldots$

4.5 Density minimum evolution of the disk gap . . . . . . . . 39

4.6 Density profile for different planet masses $\mu \ldots \ldots \ldots$ 
4.7 Density minimum of the disk gap for several planet masses . . . . . 41

5.1 Diagram of the disk with a planet . . . . . . . . . . . 44

5.2 Time evolution of the total torque $T_{d}$ exerted on the planet . . . . 46

5.3 Torque contribution from different regions in the disk . . . . . . . 47

5.4 Potential vorticity image at $t=110 P$ and at $t=400 P \ldots 49$

5.5 Long term torque evolution of a simulation . . . . . . . . . 50

6.1 Evolution of the azimuthally averaged potential vorticity . . . . . 58

6.2 Potential vorticity at $t=80 P \ldots \ldots \ldots$. . . . . . 59

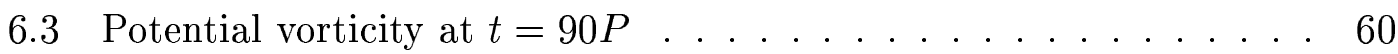

6.4 Potential vorticity at $t=100 P \ldots \ldots \ldots$. . . . . . 61

6.5 Density of a protoplanetary disk with an embedded planet . . . . 62

6.6 Shock structure in terms of Mach number .......... 63

6.7 Azimuthally averaged potential vorticity before vortices develop . . 64

7.1 Shock structure in Mach number for different sound speeds . . . . . 68

7.2 Torque evolution with constant key parameter . . . . . . . . . 69

7.3 Azimuthally averaged PV profile shortly before vortices emerge . 70

7.4 Torque evolution of several sound speed simulations . . . . . . . 79

7.5 Surface density and potential vorticity for low sound speed regime . 80

7.6 Surface density and potential vorticity for mid sound speed regime . 81

7.7 Surface density and potential vorticity for high sound speed regime $\quad 82$ 
7.8 Time for the onset of secondary instabilities with $c_{s} \ldots \ldots 3$

7.9 Azimuthally averaged potential vorticity profile and its derivatives . 84

7.10 Growth of $\Delta C \ldots \ldots \ldots \ldots$

7.11 Growth rate for $\Delta C(\mu)$ with different constant planet masses . . . 86

7.12 Slope of $\Delta C\left(c_{s}\right)$ before vortices emerge . . . . . . . . . 87

7.13 Evolution of $\eta \ldots \ldots \ldots$. . . . . . . . . . . 88

8.1 Azimuthally averaged surface density and potential vorticity . . . . 93

8.2 Shock structure in terms of Mach number . . . . . . . . . . 94

8.3 Radial component of several particles in the separatrix . . . . . 95

8.4 Potential vorticity evolution along stream lines . . . . . . . . . 96

8.5 Density evolution of a particle in the separatrix region . . . . . . 97

8.6 Dividing vorticity into its three contributions $a_{1}, a_{2}$, and $a_{3} \ldots \ldots 9$

8.7 Same as Fig. 8.5 but zoomed into shock structure . . . . . . . . 99

9.1 Four streamlines and density contours . . . . . . . . . 105

9.2 Shock structure in terms of Mach number . . . . . . . . 106

9.3 Inclination with the shock for $c_{s}=0.032$ and $c_{s}=0.05 \ldots 107$

9.4 Density $\Sigma_{1}$ in the pre-shock region . . . . . . . . 108

9.5 Shock structure in Mach number for different sound speeds . . . . . 109

9.6 Gradient of the Mach number for sound speed $c_{s}=0.032 \ldots 110$

9.7 Change of orbit due to the planet . . . . . . . . . 111 
9.8 Measured azimuthally averaged potential vorticity . . . . . . . . 112

9.9 Expected change of potential vorticity with each shock passage . . 113

10.1 Density image with two planets at $t=90 P \ldots \ldots 116$

10.2 Zoom into the density image for two planets at $t=150 P \ldots 117$

10.3 Potential vorticity of disk containing two planets at $t=90 P$. . 118

10.4 Potential vorticity of disk containing two planets at $t=150 P . .119$

10.5 Potential vorticity profiles for two planets . . . . . . . . . 120

10.6 Torque evolution on the first planet in a multi planet case . . . . 121

11.1 Radial velocity for a steady state disk . . . . . . . . . . . 124

11.2 Comparison of density with different grid resolutions . . . . . . 128

11.3 Azimuthally potential vorticity . . . . . . . . . . 129

11.4 Comparison of potential vorticity from VH1 and hybrid code . . . 130

11.5 Potential vorticity with same parameters as in Fig. 11.4 . . . . . 131

12.1 Two typical orbits in the three-body problem . . . . . . . 135

12.2 Libration periods for particles around $L_{3} \ldots \ldots$. . . . . . . 137

12.3 Libration periods of particles around $L_{4} \ldots \ldots$. . . . . . 138

12.4 Particle trajectories by solving the restricted three-body equations . 140

12.5 Snapshot of one million particles from three-body code . . . . . 143

12.6 Particle number density compared with hydrodynamic simulations . 144

12.7 Three particle orbits with an increasing planet mass . . . . . . 146 


\section{List of Tables}

7.1 Turn-on times for different initial mass ratios . . . . . . . . 76 


\section{Chapter 1}

\section{Introduction and Motivation}

Since the first discovery of extrasolar planets by Wolszczan \& Frail (1992) and Wolszczan (1994) around pulsars and then three years later with the discovery of a companion to 51 Pegasi in the peculiar 4.23-day orbit (Mayor \& Queloz, 1995), a total of 120 extrasolar planets were found as of March 2004. An updated list can be viewed at http://www.obspm.fr/encycl/encycl.html or http://exoplanets .org. The successfully applied Doppler method for extrasolar planet searches (Marcy \& Butler, 1998) is most sensitive to candidates that impart the largest reflex velocity to the host star. The velocity semiamplitude, $K$, is a function of host star and planet mass, eccentricity, and inclination angle (Marcy et al., 2000). With the Doppler technique alone, the actual planet mass $M_{p}$ remains unknown pending the determination of the orbital inclination $i$. Therefore, the planet mass is usually provided as $M_{p} \sin i$. One major surprise of these de- 
tections was the existence of giant planets at very small radii from their host stars (Marcy et al., 2000). Since then, long duration studies revealed more planets at larger distances but, still, there remains a significant accumulation of short period planets in circular orbits of $\leq 0.25$ Astronomical Units $(\mathrm{AU})$, which is well within the orbit of Mercury (0.39 AU). Despite that current detection techniques are biased towards massive planets at close orbits, this pileup at small distances appears to be genuine (Udry et al., 2003).

However, there is a lack of higher mass planets, $M_{p} \sin i>2 M_{J}$, on short period orbits $\left(P \leq 100\right.$ days), where $M_{J}$ is the mass of our Jupiter. This is the socalled period desert and remains unexplained (Udry et al., 2003). More statistical properties of over 100 planets are reviewed in Udry et al. (2003), Santos et al. (2003), and Eggenberger et al. (2004).

Several different mechanisms are postulated to explain the observation of giant planets at small orbital radii. Generally it is believed that planets form at larger distances either due to rapdi collapse through gravitational instabilities (Boss, 2001a,b; Mayer et al., 2002) or by coalescence of dust and planetesimals (Pollack et al., 1996). The latter standard model predicts that a core of $M_{p} \approx 10 M_{E}$, where $M_{E}$ is one Earth mass, would be sufficient to accrete the surrounding gas exponentially in a process called run-away accretion (Pollack et al., 1996). Both processes, gravitational collapse and coalescence of planetesimals, would lead to Jupiter mass planets. Already during the formation stage, the planet would need 
to migrate inwards by tidal interaction with disk (Lin \& Papaloizou, 1993; Ward, 1997) or by gravitational scattering with other planetesimals or planets (Murray et al., 1998; Weidenschilling \& Marzari, 1996; Rasio \& Ford, 1996). However, the latter migration scenario due to scattering is probably not strong enough to explain the short period giants and, hence, the disk-planet tidal interactions are currently the most likely process for migration.

Well before extrasolar planets were discovered, Goldreich \& Tremaine (1979, 1980) and Lin \& Papaloizou (1986a,b) speculated that tidal interactions of disks with embedded protoplanets would lead to planet migration. Ward (1997) suggested two different types of migration scenarios. One, called type I migration, would play a crucial role when the planet mass is still small. The other one (type II migration) comes into play after the planet gained enough mass to open a gap (Ward, 1997; Papaloizou \& Lin, 1984). Then the planet would be tidally locked in that gap and migrate only on a much smaller viscous time scale (Nelson et al., 2000). The discovery of extrasolar planets with a large number of short-period planets very close to the star seems to support a migration scenario since it is more likely that planet formation takes place at larger distances outside the snow line (Kornet et al., 2004, and references therein). This has generated renewed interests in the study of tidally induced planet migration. Both types of migration have been basically confirmed by non-linear numerical simulations but the calculated migration rates are typically a few orders of magnitude too short and range from 
$10^{5}$ to $10^{6}$ years (Trilling et al., 2002). This timescale is relatively short compared to the estimated life time of a disk between $10^{6}-10^{7}$ years (Hollenbach et al., 2000). If tidal interaction is the main process for migration then a planet would fall into the host star well before the disk is dispersed (Sandquist et al., 2002). Hence, the high mobility of small mass protoplanets or embryo cores of giant planets from Type I migration has presented a challenge to the formation of giant planets. It is thus imperative to explore effects that may inhibit Type I migration. One potential contribution to this process is the disk response to low mass planets, especially in the co-orbital region, where only sketchy analytical arguments have been developed. There are, however, two notable recent exceptions. Masset (2001, 2002) evaluated the co-orbital corotation torque and found that they can be a significant factor for the migration rate. He found a generic behavior of the total disk torque showing first an oscillation consistent with a librating fluid but leveling off to a constant value because of an (artificially) imposed disk viscosity. In another study Balmforth \& Korycansky (2001) noticed for inviscid flows the formation of vortices. They investigated the role of re-arranging the potential vorticity profile in the disk response to the planet and attributed them to a secondary instability in the corotation resonance.

This research shows that similar vortices may develop in the co-orbital region but for the first time they are seen and described in "brute-force" hydro simulations. Also for the first time, a connection between vortices in that region and 
the torque evolution is made. Compared to Masset (2002), the simulations confirm the generic structures produced in the disk by the tidal interactions between the planet and its surrounding flows. However, the focus is on the dynamics of the co-orbital region defined as $|\Delta r| \leq 6 r_{H}$, where $\Delta r=\left(r-r_{p}\right)$ and $r_{H}$ is the Roche lobe radius of the planet and $r_{p}$ is the radial location of the planet in the disk. The co-orbital region can be separated into several sub-regions depending on their streamline behavior (see Masset, 2002), including the horseshoe (or librating) region $|\Delta r|<r_{H}$, the separatrix region $r_{H}<|\Delta r|<\sqrt{12} r_{H}$, and the streaming region $|\Delta r|>\sqrt{12} r_{H}($ see $\S 12)$.

In $\S 2$ the numerical model is described in detail including a discussion about the influence of a smoothing factor for the planet potential in $\S 3$ and the general density evolution of the disk $(\S 4)$. It is found that the torque evolution $(\S 5)$ can be divided into three phases: in Phase I, the total torque is negative and smooth as in Masset (2002), modulated by a period that is similar to the libration period $\tau_{l i b} \sim \sqrt{4 / 27 \mu}$, where $\mu$ is the planet mass; Phase II, the total torque shows very large amplitudes and fast oscillations with a quasi-period of a few orbits; and phase III, where the torque variations switch to a lower frequency but with higher amplitude. Investigating the potential vorticity in $\S 6$ revealed that vortices are produced exactly when the phase transition of the torque occurs. The principal and derived variables are studied on particle paths as described in $\S 8$. The vortex production is investigated in detail and how it depends on sound speed and planet 
mass $(\S 7)$. Furthermore, multiple planet systems $(\S 10)$ are briefly analyzed and a comparison is made with the restricted three-body problem in $\S 12$. Chapter 9 describes an analytical estimate for the potential vorticity jump and correlates it with the measured jump in simulations. Finally, Chapter 11 compares the applied simulation code used for this work with standard tests and a different hydro-code VH1. 


\section{Chapter 2}

\section{Model Description}

\section{$2.1 \quad$ Numerical Scheme}

The hydrodynamic equations are solved using a finite difference scheme on a fixed cylindrical Eulerian $(r, \phi)$ grid in corotating coordinates. The protoplanetary disk is assumed as thin and can be described by the inviscid two-dimensional Euler equations with vertically integrated quantities.

The corotating frame is used such that the positions of the central star and the planet are fixed at $\left(r_{*}, \phi_{*}\right)=(0, \pi)$ and $\left(r_{p}, \phi_{p}\right)=(1,0)$ respectively. The planet is on a fixed circular orbit at $r_{p}=1$. The primary variables are surface density $\Sigma$ and velocity vectors $\left(v_{r}, v_{\phi}\right)$. All other parameters derive from there (see Section $\S 2.4$ on the equation of state).

The scheme to solve the hydrodynamic equations applies dimensionally splitting 
where the radial and angular equation are treated separately as

$$
\begin{aligned}
& \frac{\partial \boldsymbol{U}}{\partial t}+\frac{\partial \boldsymbol{F}}{\partial r}=\boldsymbol{S} \\
& \frac{\partial \boldsymbol{U}}{\partial t}+\frac{\partial \boldsymbol{G}}{\partial \phi}=0 .
\end{aligned}
$$

For a definition of $\boldsymbol{U}, \boldsymbol{F}, \boldsymbol{G}$ and $\boldsymbol{S}$, see Section $\S 2.2$. The finite difference equations are solved such that scalar quantities are defined at cell centers, while vector quantities are located at cell interfaces. An operator-splitting is employed in which one time step is split into two sub-steps, dealing with the $r$ and $\phi$ direction separately in alternating order. The integrations are called in the sequence of radial, angular, angular, and radial where the result from each sweep is used as input for the next one.

The numerical integration in flux form is written as

$$
\begin{aligned}
\boldsymbol{U}_{i}^{\prime} & =\boldsymbol{U}_{i}-\lambda\left[\boldsymbol{F}_{i+1 / 2}-\boldsymbol{F}_{i-1 / 2}\right]+\Delta t \boldsymbol{S} \\
\lambda & =\Delta t / \Delta r
\end{aligned}
$$

for the radial integration. For the angular integration we have

$$
\begin{aligned}
\boldsymbol{U}_{j}^{\prime} & =\boldsymbol{U}_{j}-\lambda\left[\boldsymbol{G}_{j+1 / 2}-\boldsymbol{G}_{j-1 / 2}\right] \\
\lambda & =\Delta t / r \Delta \phi
\end{aligned}
$$


where $i$ and $j$ are the cell center of $r$ and $\phi$ respectively and $i, j \pm 1 / 2$ is defined on the cell edges.

The fluxes $\boldsymbol{F}, \boldsymbol{G}$ are calculated with a hybrid method of the second order LaxWendroff and the first order Lax-Friedrich scheme. Hence, the simulation code is addressed as a hybrid code from here on. The code has been well tested before by Li et al. (2001). The two schemes are combined in their fluxes $F$ as

$$
\boldsymbol{F}=\alpha \boldsymbol{F}^{L F}+(1-\alpha) \boldsymbol{F}^{L W}
$$

with $0 \leq \alpha \leq 1$. The weight $\alpha$ is

$$
\alpha=\min \left[\frac{\left|\left(v_{r}\right)_{i+1}-\left(v_{r}\right)_{i-1}\right|}{c_{i}}, 1\right]^{2}
$$

where $c_{i}$ is the local sound speed in grid cell $i$ and $v_{r}$ is the radial velocity. For the angular sweep, $\boldsymbol{F}$ is replaced by $\boldsymbol{G}, i$ by $j$ and $v_{r}$ by $v_{\phi}$ according to the integration sweep in the dimensionally split scheme. More details about the hybrid scheme can be found in Li et al. (2001). Information on how to calculate the standard Lax-Friedrich and Lax-Wendroff fluxes is available in most books on computational gas-dynamics (e.g. Laney, 1998).

Another basic algorithm is included based on FARGO (Fast Advection in Rotating Gaseous Objects) by Masset (2000) with some changes by Li et al. (2001). This method applies a local co-moving frame for the $\phi$ sweeps to avoid the strin- 
gent time constraint from the inner boundary of the disk. This method reduces the computing time greatly over a standard two-dimensional method since $v_{\phi}$ is much greater than the sound speed over the whole disk.

Several numerical grid resolutions are used to perform the calculations. The standard resolution contains 300 radial zones and 1200 angular zones. The radial zones are distributed between $r_{\min }=0.4$ and $r_{\max }=2.0$. Higher resolution grids up to $\left(N_{r}, N_{\phi}\right)=(600,2400)$ are also applied to test the resolution dependence of the results presented here. The ratio of radial zones versus angular zones is always chosen as $1: 4$ since the radial extent versus $2 \pi$ is also a factor of $1 / 4$. That gives us almost square grid cells at $r=1$, where the planet is located.

An artificial viscosity is not included although some numerical viscosity is inevitable and necessary to handle phenomena such as shocks.

All coordinates and variables have been normalized to the Keplerian velocity of the planet at $r_{p}=1$. All other quantities derive from that.

\subsection{Hydrodynamic Equations}

The governing equations describing the flow are the equation of continuity

$$
\frac{\partial \Sigma}{\partial t}+\nabla \cdot(\Sigma \boldsymbol{v})=0
$$


together with the equation of motion which takes the form

$$
\frac{\partial(\Sigma \boldsymbol{v})}{\partial t}+\nabla \cdot(\Sigma \boldsymbol{v} \boldsymbol{v})=-\Sigma \nabla \Phi-\nabla P
$$

where $\Sigma$ is the vertically integrated surface density, $P$ the pressure, $\Phi(r, \phi)$ the gravitational potential, and $\boldsymbol{v}=\left(v_{r}, v_{\phi}\right)$ is the velocity vector.

An energy equation is not required since an isothermal equation of state is used where the pressure is given by

$$
P=\Sigma c_{s}^{2}
$$

Consequently, the Euler equations in cylindrical coordinates become

$$
\frac{\partial \boldsymbol{U}}{\partial t}+\frac{\partial \boldsymbol{F}}{\partial r}+\frac{\partial \boldsymbol{G}}{\partial \phi}=\boldsymbol{S}
$$

where

$$
\begin{aligned}
& \boldsymbol{U}=\left(\begin{array}{c}
r \Sigma \\
r \Sigma v_{r} \\
r^{2} \Sigma v_{\phi}
\end{array}\right), \quad \boldsymbol{F}=\left(\begin{array}{c}
r \Sigma v_{r} \\
r \Sigma v_{r}^{2} \\
r^{2} \Sigma v_{r} v_{\phi}
\end{array}\right), \quad \boldsymbol{G}=\left(\begin{array}{c}
\Sigma v_{\phi} \\
\Sigma v_{r} v_{\phi} \\
r \Sigma v_{\phi}^{2}+r P
\end{array}\right), \text { and } \\
& \boldsymbol{S}=\left(\begin{array}{c}
0 \\
-r d P / d r+\Sigma v_{\phi}^{2}-r \Sigma d \Phi / d r \\
-r^{2} \Sigma d \Phi / r d \phi
\end{array}\right) .
\end{aligned}
$$


The gravitational potential $\Phi(r, \phi)$ is due to the central star at $\left(r_{*}, \phi_{*}\right)=(0,0)$ and the planet at $\left(r_{p}, \phi_{p}\right)=(1, \pi)$ in normalized coordinates. The potential can be written as

$$
\Phi=-\frac{G M_{*}}{r}-\frac{G M_{p}}{\left(r^{2}+r_{p}^{2}-2 r r_{p} \cos \left(\phi-\phi_{p}\right)+s^{2}\right)^{1 / 2}}+\frac{G M_{p} r \cos \left(\phi-\phi_{p}\right)}{r_{p}^{2}}
$$

where the first term is from the central star with mass $M_{*}$, the second term from the companion with mass $M_{p}$. The third indirect term accounts for the acceleration of the rotating coordinate system (Bryden et al., 1999). The last term can be neglected since it scales only with the star/planet mass ratio $\mu=$ $10^{-4}$ everywhere in the disk. Hence, it is small compared to the other terms. A gravitational softening radius $s$ is also included for avoiding a numerical singularity at the position of the planet. The gravitational potential of the star does not require a smoothing factor since that area is excluded from the simulations by the inner boundary.

The Euler equations are not asking for a potential but for the force components $F_{r}$ and $F_{\phi}$, which can be derived from $\boldsymbol{F}=-\nabla \Phi$. In normalized units, they are

$$
\begin{aligned}
& F_{r}=-\frac{1}{r^{2}}-\frac{\mu\left[r-r_{p} \cos \left(\phi-\phi_{p}\right)\right]}{d^{3}}-\frac{\mu \cos \left(\phi-\phi_{p}\right)}{r_{p}^{2}} \\
& F_{\phi}=-\frac{\mu\left[r_{p} \sin \left(\phi-\phi_{p}\right)\right]}{d^{3}}+\frac{\mu \sin \left(\phi-\phi_{p}\right)}{r_{p}^{2}}
\end{aligned}
$$


where $d$ is the distance of the surface element (i.e. grid cell) to the planet

$$
d=\left(r^{2}+r_{p}^{2}-2 r r_{p} \cos \left(\phi-\phi_{p}\right)+s^{2}\right)^{1 / 2} .
$$

\subsection{Angular Momentum Conservation in Rotat- ing Coordinates}

In the following, the coordinate system will be transformed to a corotating system with $\tilde{\omega}=\omega-\Omega$. The rotation is around the z-axis where $\boldsymbol{\Omega}=(0,0, \Omega)$ is the corotation vector. For all simulations, $\Omega=1$. Defining the angular velocity as $\omega=v_{\phi} / r$ in the inertial frame, and $\tilde{v}_{\phi}=v_{\phi}-r$ as the velocity in the rotating frame, then the momentum part from the Euler equation (2.10) turns into

$$
\begin{gathered}
\frac{\partial\left(\Sigma v_{r}\right)}{\partial t}+\nabla \cdot\left(\Sigma v_{r} \boldsymbol{v}\right)=\Sigma r(\omega+\Omega)^{2}-\frac{\partial P}{\partial r}-\Sigma \frac{\partial \Phi}{\partial r} \\
\frac{\partial\left(\Sigma r^{2} \omega\right)}{\partial t}+\nabla \cdot\left(\Sigma r^{2} \omega \boldsymbol{v}\right)=-2 \Sigma v_{r} \Omega r-\frac{\partial P}{\partial \phi}-\Sigma \frac{\partial \Phi}{\partial \phi}
\end{gathered}
$$

where the momentum equation is split into the radial and angular component. We note here that the angular momentum equation (2.19) includes the Coriolis force $F_{c}=-2 \Sigma \boldsymbol{\omega} \times \boldsymbol{v}$ explicitly as a source term on the right hand side. However, that does not conserve angular momentum if treated numerically with a finite 
differencing scheme (Kley, 1998). Nevertheless, Eq. (2.19) can be rewritten as

$$
\frac{\partial\left(\Sigma r^{2}(\omega+\Omega)\right)}{\partial t}+\nabla \cdot\left(\Sigma r^{2}(\omega+\Omega) \boldsymbol{v}\right)=-\frac{\partial P}{\partial \phi}-\Sigma \frac{\partial \Phi}{\partial \phi}
$$

which includes the Coriolis term now implicitly. Kley (1998) showed that this form of Eq. (2.20) will lead to correct results in the corotating frame in terms of angular momentum conservation.

To summarize, treating the Euler equation with a conservative angular momentum scheme, the vector identities $\boldsymbol{U}, \boldsymbol{F}, \boldsymbol{G}, \boldsymbol{S}$ from the principal equation (2.12) will change into

$$
\begin{aligned}
\boldsymbol{U} & =\left(\begin{array}{c}
r \Sigma \\
r \Sigma v_{r} \\
r^{2} \Sigma v_{\phi}
\end{array}\right), \quad \boldsymbol{F}=\left(\begin{array}{c}
r \Sigma v_{r} \\
r \Sigma v_{r}^{2} \\
r^{2} \Sigma v_{r} v_{\phi}
\end{array}\right), \quad \boldsymbol{G}=\left(\begin{array}{c}
\Sigma \tilde{v}_{\phi} \\
\Sigma v_{r} \tilde{v}_{\phi} \\
r \Sigma v_{\phi} \tilde{v}_{\phi}+r P
\end{array}\right), \text { and } \\
& \boldsymbol{S}=\left(\begin{array}{c}
-r d P / d r+\Sigma v_{\phi}^{2}-r \Sigma d \Phi / d r \\
-r^{2} \Sigma d \Phi / r d \phi
\end{array}\right),
\end{aligned}
$$

where $\tilde{v}_{\phi}$ is the angular velocity in corotating coordinates. Note, that $\tilde{v}_{\phi}$ enters only in $\boldsymbol{G}$. This is due to the angular momentum conservative treatment Eq.(2.20) which can be rewritten in terms of $\tilde{v}_{\phi}+r=v_{\phi}$ since the corotation velocity $\Omega=1$. 


\subsection{Equation of State}

Dissipation, caused by some type of artificial viscosity or viscosity from nonlinear shocks, can cause local heating. If no cooling mechanism is provided, the dissipation would adiabatically heat up the disk. Instead, we assume the disk is able to radiate away all excess thermal energy from dissipation and remain geometrically thin. The situation is adequately approximated by assuming a polytropic or isothermal equation of state (Lin \& Papaloizou, 1985). Here, an isothermal equation of state (EOS) is chosen. Hence, the disk gas temperature and the sound speed remains unchanged and is treated as if all excess energy is instantly radiated away and every disk element is enshrouded in an external heat bath, i.e. radiation from the central star. Each gas element keeps the same temperature throughout the simulation. The sound speed $c_{s}$ and temperature $T$ are assumed independent of $r$ and constant throughout the disk.

Since all parameters are used in normalized coordinates, a relationship between the normalized sound speed and the real temperature is useful. Temperature $T$ can be calculated from sound speed $c_{s}$ by

$$
c_{s}=\sqrt{\frac{\gamma \mathcal{R} T}{M_{H}}}
$$

where $\gamma$ is the adiabatic coefficient and $M_{H}$ is the molecular weight of the gas

in $[\mathrm{kg} / \mathrm{mol}]$. For atomic gas, like hydrogen, the value for $\gamma=5 / 3$ for adiabatic 
equation of state. Since we chose an isothermal equation of state here, $\gamma$ can be set to $\gamma=1$. The universal gas constant is $\mathcal{R}=8.314 \mathrm{~J} / \mathrm{mol} \mathrm{K}$. The molecular weight of hydrogen is $M_{H}=m_{H} N_{a}=0.001 \mathrm{~kg}$, where $m_{H}$ is the mass of a single hydrogen atom and $N_{a}$ is the Avogadro constant.

In an accretion disk the sound speed versus scale height $H$ is

$$
\frac{c_{s}}{v_{K}}=\frac{H}{r}
$$

with the Keplerian velocity of

$$
v_{K}=\sqrt{\frac{G M_{*}}{r}} .
$$

Putting everything together, we find

$$
T=\frac{c_{s}^{2} M_{H}}{\gamma \mathcal{R}}=\frac{107000}{\gamma r[\mathrm{AU}]}\left(\frac{c_{s}}{v_{K}}\right)^{2} .
$$

For e.g. a $c_{s} / v_{K}=0.1$ and $\gamma=5 / 3$, the temperature at $1 \mathrm{AU}$ will be $642 \mathrm{~K}$. Fig. 2.1 illustrates the dependence of temperature on sound speed for adiabatic and isothermal EOS respectively. 


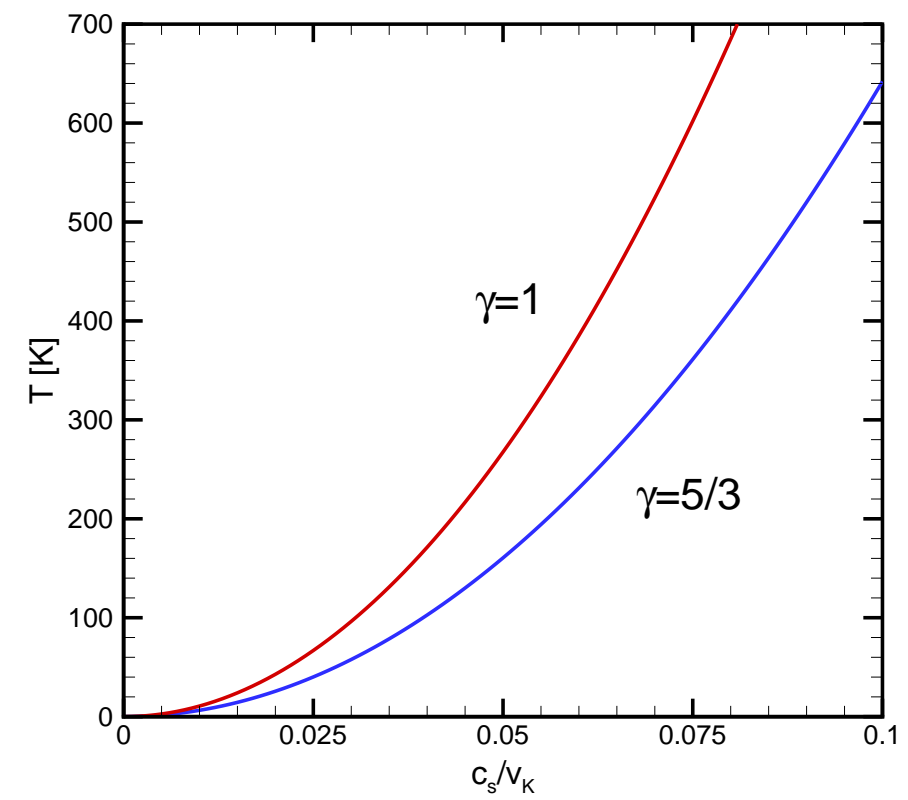

Figure 2.1: Sound speed temperature relation at $1 \mathrm{AU}$ for isothermal equation of state $(\gamma=1$, red $)$ and adiabatic $\operatorname{EOS}(\gamma=5 / 3$, blue $)$.

\subsection{Initial and Boundary Conditions}

If not otherwise noted in the text, the standard model has a planet/star mass ratio of $\mu=M_{p} / M_{*}=10^{-4}$. The Roche lobe (or Hill) radius is $r_{H}=r_{p}(\mu / 3)^{1 / 3}$. The protoplanet's semi-major axis is set to $r_{p}=1$. The surface density is $\Sigma(r) \propto$ $r^{-\beta}$ where $\beta=0$ for a constant density. Typically $\beta=3 / 2$ was chosen to obtain a constant potential vorticity (see below). The initial conditions for a stable steadystate disk are therefore

$$
\begin{aligned}
\Sigma(r) & =r^{-\beta} \\
v_{r} & =0
\end{aligned}
$$




$$
v_{\phi}=\sqrt{1+c_{s}^{2} r^{2} \frac{\Sigma^{\prime}(r)}{\Sigma(r)}} \sqrt{\frac{1}{r}}
$$

where $\Sigma^{\prime}(r)=d \Sigma(r) / d r$. The equations above for the steady-state conditions for $v_{\phi}$ are valid for isothermal equations of state only. Usually a density profile was chosen so that the ratio of vorticity to surface density (i.e. potential vorticity) has a flat radial profile

$$
\zeta=(\nabla \times \boldsymbol{v}) \hat{z} / \Sigma \approx \text { const }
$$

which is given if $\beta=3 / 2$. However, $\zeta$ has a small deviation from a precisely constant value since the density gradient imposes a pressure gradient on the disk. The radial pressure gradient is small but slightly counteracts the gravitational force from the central star. Hence, the $v_{\phi}$ velocity is reduced from a Keplerian to an almost Keplerian velocity by a few percent.

With this choice of initial condition, the generation of inflection points is avoided due to the rearrangement of the $\zeta$ distribution as it is carried by the stream lines in the horseshoe region. Other runs with a strong initial potential vorticity gradient were also studied with the conclusion that the results presented here hold for them too.

The disk boundaries are open at $r_{\min }=0.4$ and $r_{\max }=2.0$. The open boundaries are such that a minimal amount of waves is reflected back from the boundary. This is achieved by extrapolating the initial conditions Eq. (2.26-2.28) into the 
ghost cells and keeping them at the same value throughout the simulation. Since the full azimuthal extent of the disk is simulated, the azimuthal boundaries are periodic.

The planet's gravitational potential is softened by an approximate three-dimensional treatment which gives an effective softening distance that is roughly two times $r_{H}$ (see $\S 3.2$ ). Although there is some accumulation of gas near the planet, accretion onto it is not allowed. This boundary condition is equivalent to that of sufficiently low-mass protoplanets with quasi hydrostatic envelopes (Pollack et al., 1996).

One key difference between the simulations here and previous studies (Lubow et al., 1999; Bryden et al., 1999, 2000; Masset, 2001, 2002; D'Angelo et al., 2002, 2003; Kley, 1999; Kley et al., 2001) is that the simulations here are performed at the inviscid limit, i.e., a viscosity term is not explicitly included, though numerical viscosity is inevitable and is needed to handle shocks. In addition, the simulations here tend to be of higher resolution. With a radial $N_{r}=300$ (some runs go up to $\left.N_{r}=600, N_{\phi}=2400\right)$ and $0.4 \leq r \leq 2$, the diameter of the Hill sphere of a planet with $\mu=10^{-4}$ is resolved by at least $\sim 12$ cells in each direction. Using a nested-grid technique, D'Angelo et al. (2002) have also made very high resolution simulations, though their simulations include an explicit viscosity. 


\section{Chapter 3}

\section{Smoothing Factor}

\subsection{Effects of the Smoothing Factor}

For preventing a numerical singularity near the planet, Kley (1999) introduced a softening distance $s$ for the gravitational potential of the planet $\Psi_{p}$ where he defined the smoothing length as $1 / 5$ of the Roche radius $r_{H}$. Other authors (e.g. Masset, 2002) defined $s$ as a fraction of the disk scale height $H$. Masset (2002) discussed that $0.6 H$ would give a close approximation to three-dimensional simulations. In Koller \& Li (2003) the effects of the smoothing factor on the non-linear disk evolution is discussed in three respects. Fig. 3.1 describes how the smoothing factor changes the potential within the Roche lobe.

(1) Surface density profile: Fig. 3.2 compares the azimuthally averaged density at $100 P$ for the standard model. For $s=0.4 r_{H}, 0.6 r_{H}$ and $1.0 r_{H}$ generally shows 
that there are two density "dips", one on each side of the planet at a distance of $\sim 3.8 r_{H}$, which is roughly $2 H$ for $c_{s}=0.07$, usually develop. This distance is larger than the often quoted $\sim \pm H$ where the pileup of Lindblad resonances (LRs) is believed to occur. This is probably due to the waves propagating further in the inviscid flows. There is a slight shift of the dips further outwards as $s$ increases. This can be attributed to the fact that as the driving by the planet is progressively weakened, so is the amplitude of the waves. Even after $t=100 P$ the density is still evolving. Hence, a steady-state has not been achieved yet.

(2) Vorticity distribution: Fig. 3.3 shows the vorticity $[=\hat{z} \cdot(\nabla \times \boldsymbol{u})]$ distribution in $(r, \phi)$ plane at $t=20 P$ for two different smoothing factors. A deeper planet potential makes the Roche lobe flow unsteady, out of which vortices are continuously "shed". These vortices are marked as black "spots", indicating negative vorticity (compared to the background). Hence, these are anticyclones which are propagating in the disk (both azimuthally and radially). Such vortices are observed in the run with $s=0.4 r_{H}$ as well (not shown here). Furthermore, all vortices are high density/pressure regions. They continuously interact with each other by merging and shearing. Also, they collectively cause the disk density to evolve completely differently from the viscous or mildly driven cases.

(3) Torques on the planet: Ward (1997) showed that there exists a mismatch between the outer and inner Lindblad torques so that the planet should always migrate inward. This has led to an important problem for small mass planets where 


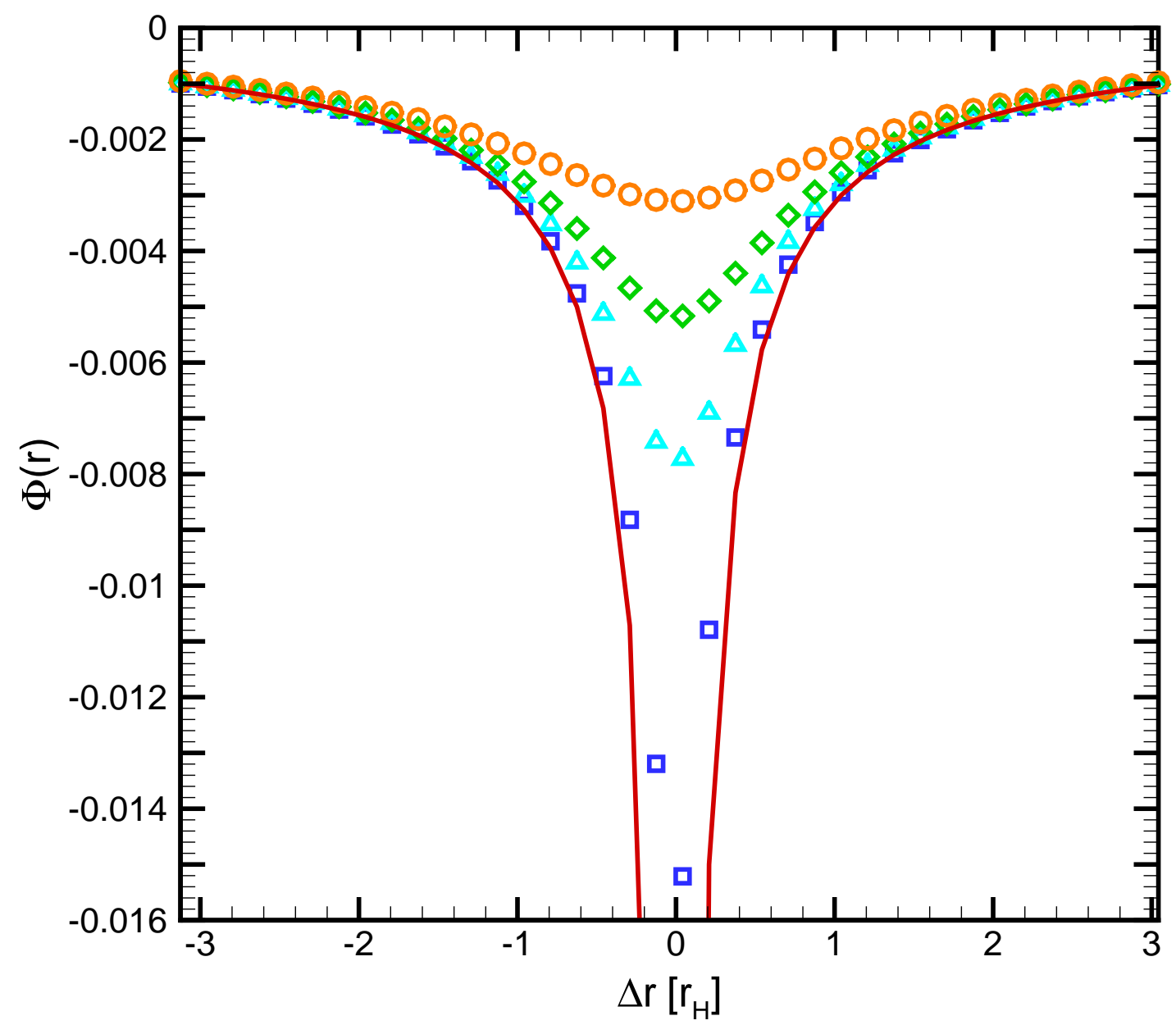

Figure 3.1: The potential of the planet with several smoothing factors $s$. The line denotes the potential without a smoothing factor. Each grid cell, with a resolution of $\left(N_{r}, N_{\phi}\right)=(300,1200)$, shows a symbol. The smoothing factors are: $s=r_{H}$ (circles), $s=0.6 r_{H}$ (diamonds), $s=0.4 r_{H}$ (triangles), and $s=0.2 r_{H}$ (squares). The potential within the Roche lobe for $s=0.2 r_{H}$ is at least twice as deep as with other smoothing factors. 


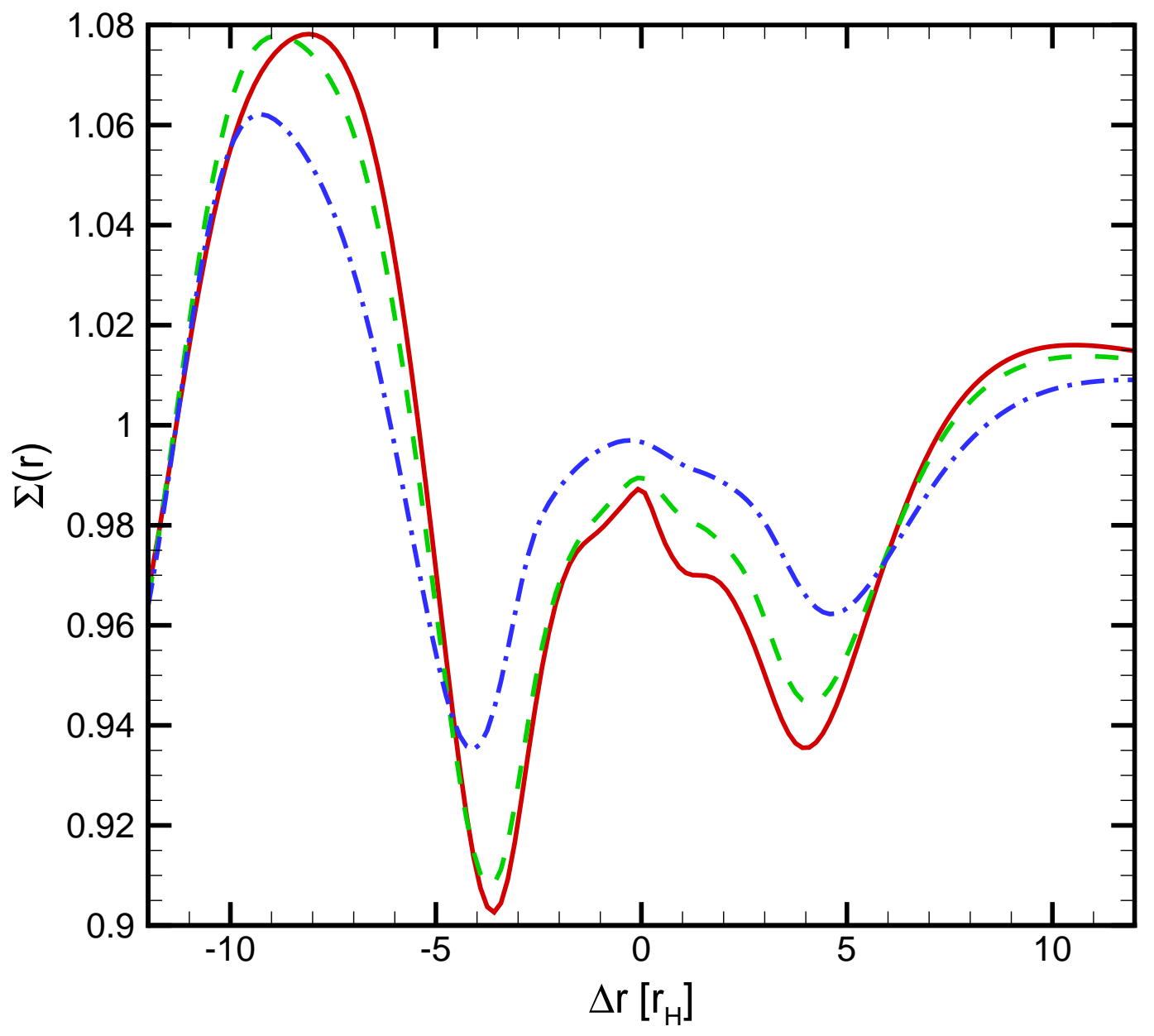

Figure 3.2: The azimuthally averaged density profile at $t=100 P$ for different smoothing lengths $s=0.4 r_{H}$ (red solid), $s=0.6 r_{H}$ (green dashed), and $s=r_{H}$ (blue dash-dotted). 

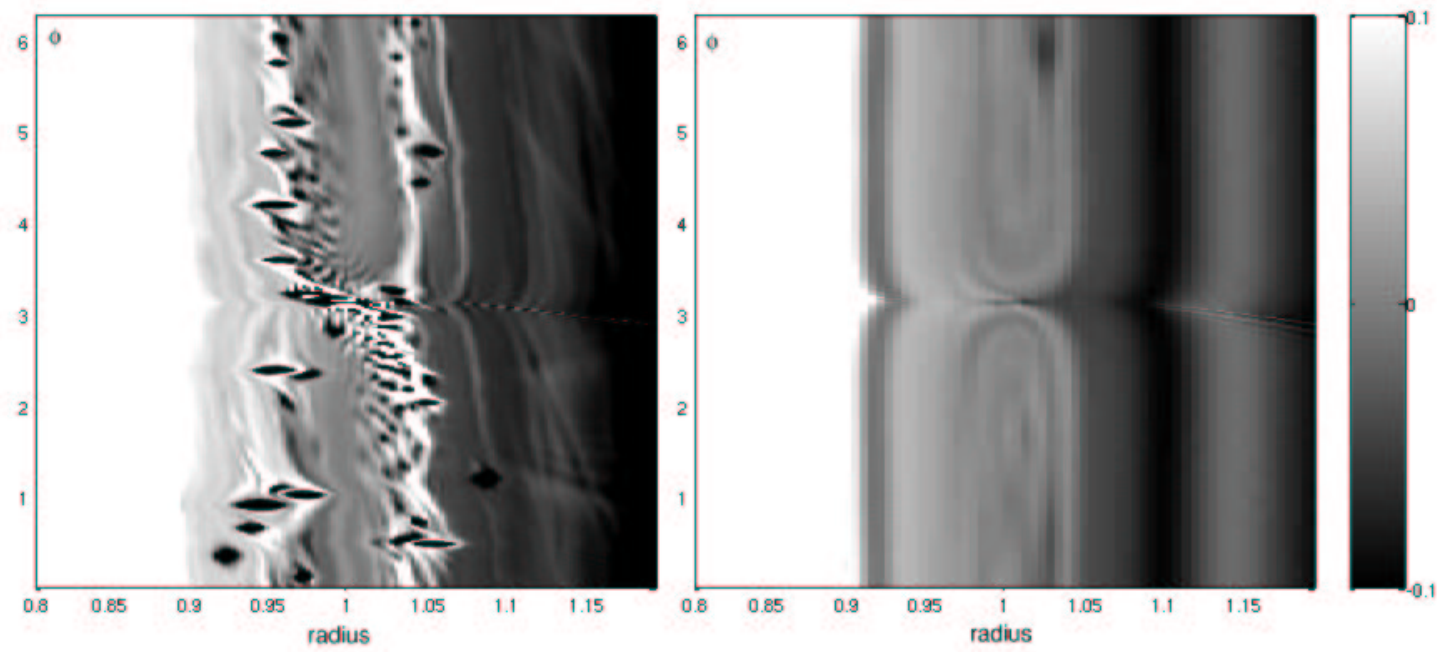

Figure 3.3: Vorticity in $(r, \phi)$ plane at $t=20 P$ from a high resolution $(600 \mathrm{x} 2400)$ simulation with $s=0.2 r_{H}$ (left) and $s=r_{H}$ (right). Gray-scale indicates the vorticity (subtracted by a constant). For $s=0.2 r_{H}$, very distinct vortices (black spots) have formed out of the Roche lobe region, whereas for $s=r_{H}$, the flow is smooth. Vortices like that are also observed with $s=0.4 r_{H}$ (though fewer in number compared with the $s=0.2 r_{H}$ case, not shown here). Vorticity mixing is clearly visible in both cases. The planet is located at $(1, \pi)$. 
he found the migration timescale is too short (Type I migration). The contribution to the total torques from the co-orbital region, however, has been a complex subject, partly due to the fact that the flow becomes nonlinear. Intuitively, flow which comes very close to the planet (i.e., at the boundary separating the circulating and librating regions) tends to give the planet the largest "kicks", causing the planet to change its semi-major axis and eccentricity.

Fig. 3.4 shows the azimuthally averaged torque on the planet. It describes the smooth Lindblad torques far away from the planet (positive/negative from inner/outer disk). The torques are clearly dominated by the co-orbital region within $\pm H$ around the planet. Large variations are seen for the $s=0.4 r_{H}$ case, mostly caused by the vortices repeatedly passing by the planet.

\subsection{Three-Dimensional Approximation}

Here, a different approach for the smoothing factor $s$ is applied, which is described below.

In two-dimensional disk simulations, all quantities are integrated over the disk scale height $H$, including the gravitational force of the planet. A careful inspection of the planet potential $\Psi_{p}$ shows that the gravitational force of the planet is weaker in three-dimensional simulations than those generated in a two-dimensional disk simulation and the following method accounts for that. 


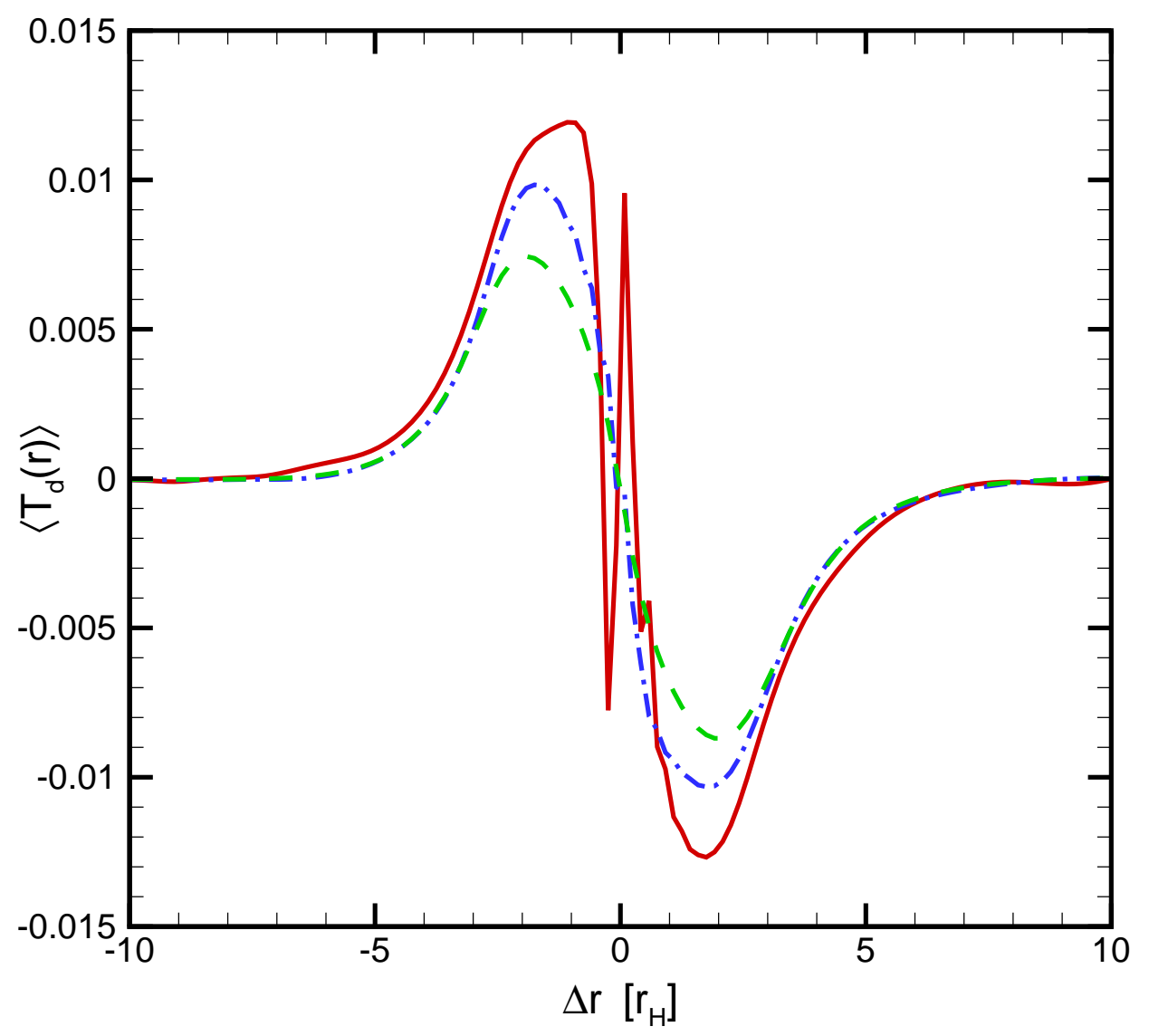

Figure 3.4: The azimuthally averaged torque at $t=100 P$ with varying smoothing $s=0.4 r_{H}$ (red), $s=0.6 r_{H}$ (blue dash-dotted), and $s=r_{H}$ (green dashed). 
The planetary force components are in three dimensions

$$
\begin{aligned}
d F_{r} & =-\frac{G M_{p}\left(r-r_{p} \cos \left(\phi-\phi_{p}\right)\right)}{d^{3}} \rho(r, \phi, z) r d r d \phi d z \\
d F_{\phi} & =-\frac{G M_{p} r_{p} \sin \left(\phi-\phi_{p}\right)}{d^{3}} \rho(r, \phi, z) r d r d \phi d z \\
d F_{z} & =-\frac{G M_{p} z}{d^{3}} \rho(r, \phi, z) r d r d \phi d z .
\end{aligned}
$$

where $d(r, \phi, z)=\left(r^{2}+r_{p}^{2}-2 r r_{p} \cos \left(\phi-\phi_{p}\right)+z^{2}\right)^{1 / 2}$ is the distance from the computational cell to the planet. We assume the disk material is distributed with a Gaussian function with scale height $H$

$$
\rho(r, \phi, z)=\rho_{0}(r, \phi) \exp \left(-z^{2} / 2 H^{2}\right)
$$

If the disk has a constant sound speed, $H$ is a function of $r$ since

$$
\frac{c_{s}}{v_{K}}=\frac{H}{r}
$$

With the Keplerian velocity $v_{K}=\sqrt{1 / r}$, we obtain

$$
H=c_{s} r^{3 / 2}
$$


By integrating over the disk height, the force components in the plane become

$$
\begin{aligned}
d F_{r} / r d r d \phi & =-\frac{G M_{p}\left(r-r_{p} \cos \left(\phi-\phi_{p}\right)\right) \Sigma(r, \phi)}{H(2 \pi)^{1 / 2}} \int_{-\infty}^{+\infty} \frac{e^{-z^{2} / 2 H^{2}}}{d^{3}} d z \\
d F_{\phi} / r d r d \phi & =-\frac{G M_{p} r_{p} \sin \left(\phi-\phi_{p}\right) \Sigma(r, \phi)}{H(2 \pi)^{1 / 2}} \int_{-\infty}^{+\infty} \frac{e^{-z^{2} / 2 H^{2}}}{d^{3}} d z
\end{aligned}
$$

Equations $(3.7,3.8)$ are computed numerically for $z \in[-6 H,+6 H]$ as the integration limits. This time consuming integration is only done once at the beginning of the simulation and multiplied to the gravitational potential as a correction factor, i.e. $C(r, \phi) \Psi_{p}$, with

$$
C(r, \phi)=2 \alpha \int_{0}^{+6 H} \frac{e^{-z^{2} / 2 H^{2}}}{d^{3}} d z
$$

where $\alpha=\left[r^{2}+r_{p}^{2}-2 r r_{p} \cos \left(\phi-\phi_{p}\right)\right]^{3 / 2} / H(2 \pi)^{1 / 2}$.

$C(r, \phi)<1$ at every point in the grid which means that the gravitational force of the planet is reduced. Fig. 3.5 shows the value of $C(r, \phi)$ near the planet. However, a smoothing factor is still necessary to avoid a numerical singularity at the planet but it can be a very small number. Still, an additional smoothing factor of typically $s=r_{H}$ was applied.

Fig. 3.6 and Fig. 3.7 give a comparison of the forces used in a standard twodimensional treatment versus the approximated three-dimensional version. Close to the planet the standard force is overemphasized by more that a factor of two. 


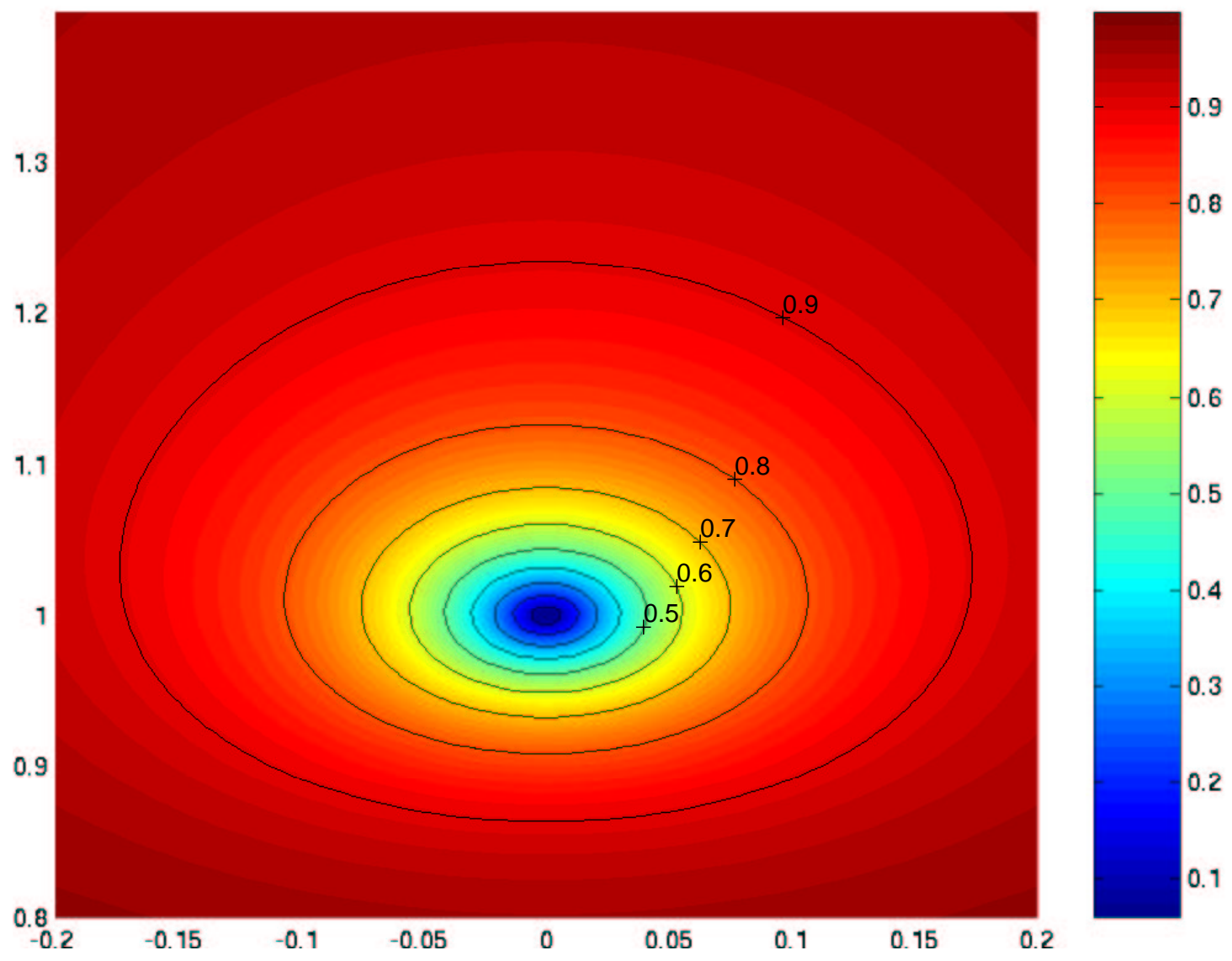

Figure 3.5: Correction Factor for the three-dimensional approximation around the planet. The planet is located in the center at $r_{p}=1$ and $\phi_{p}=0$. The correction factor is shown with a color scale in this contour plot. Contour lines are in steps of 0.1 


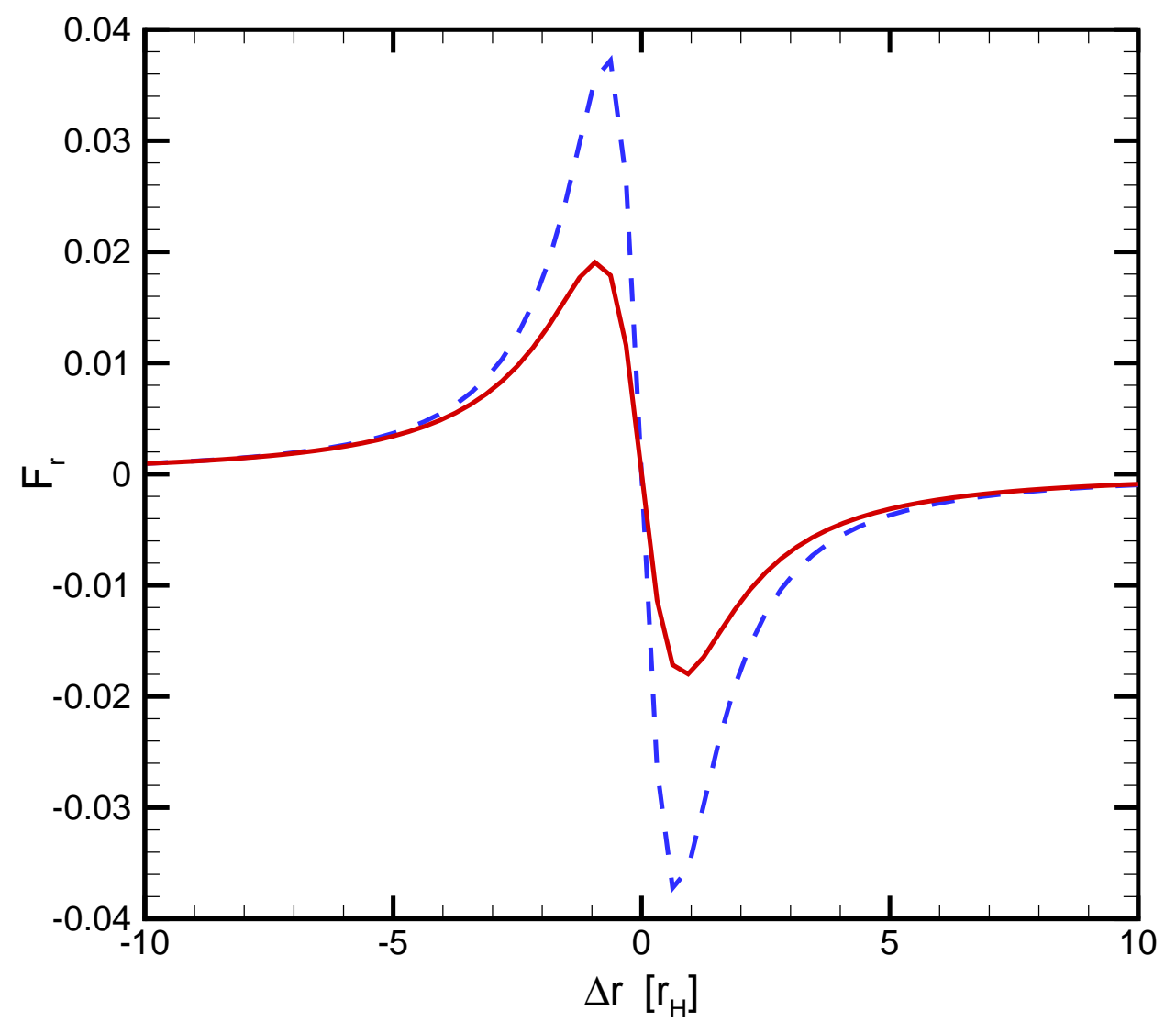

Figure 3.6: Comparison of the original radial force component with the new corrected version. Here $F_{r}$ is plotted of a planet mass $\mu=10^{-4}$ cutting radially through the disk. The standard two-dimensional force (dashed line) is about twice as strong close to the planet as the force corrected with an approximated threedimensional treatment (solid line). 


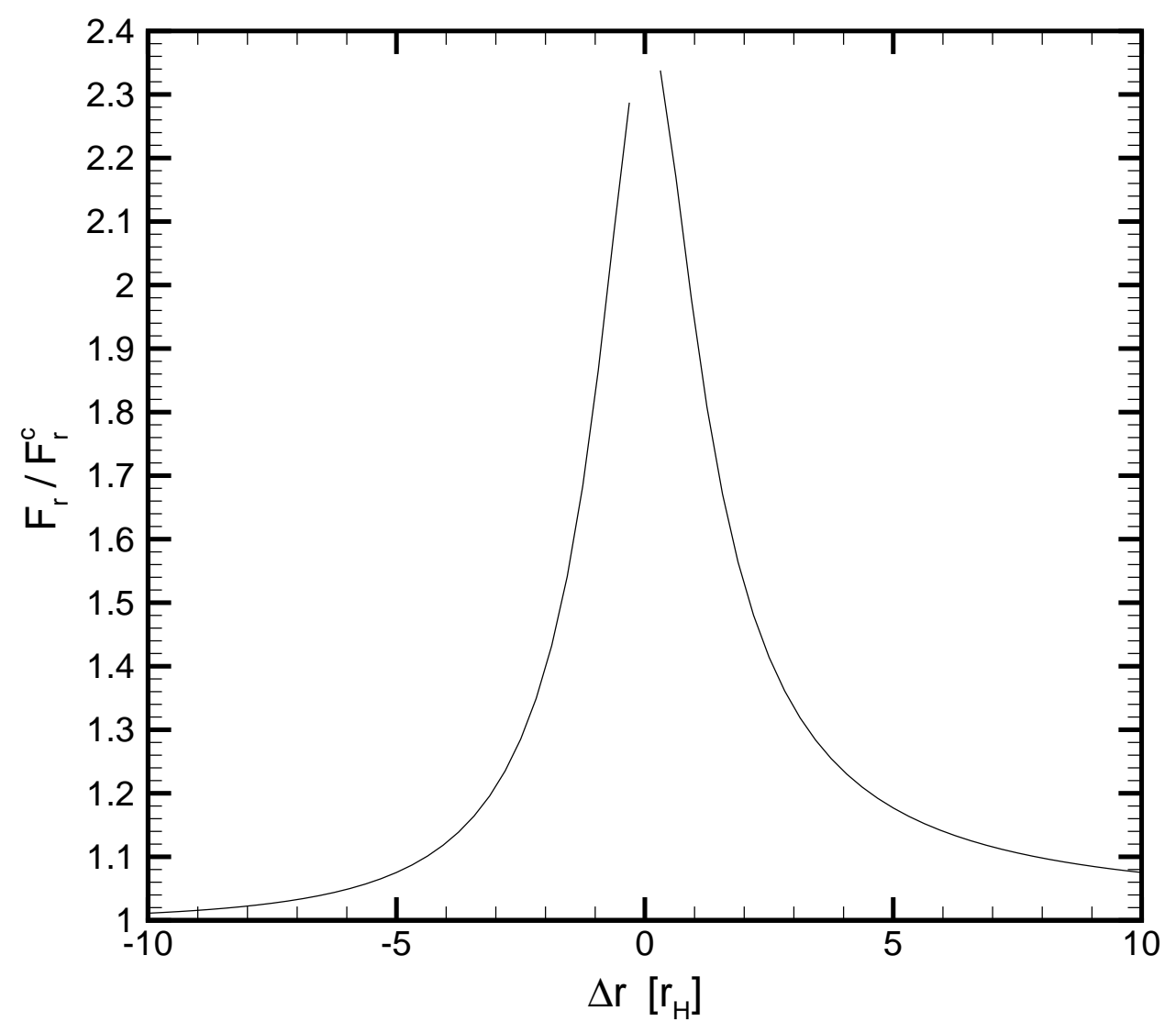

Figure 3.7: Standard planetary force ove the approximated three-dimensional force. Close to the planet the force is more than twice overestimated by the standard two-dimensional treatment. A smoothing factor is still necessary and $s=r_{H}$ was applied here. 


\section{Chapter 4}

\section{Density Evolution and Gap}

\section{Formation}

Planets have not only the ability to accrete gas in their immediate vicinity, they can also strongly expel material over a wide range in radius as a result of their tidal forces. To focus on the tidal disk-planet interactions, a non-accreting planet is considered. Any surface density enhancement or reduction is entirely due to the repulsive tidal forces from the protoplanet. Fig. 4.1-4.3 show the surface density of the disk at different times $(t=50,200,500 P)$. The tidal interactions start clearing a gap but since the co-orbital region is less affected by the tidal forces, only two density channels are cleared in the beginning.

In Fig. 4.4 the azimuthally averaged density $\langle\Sigma\rangle$ is shown at a late stage of $t=500 P$ for a planet mass $\mu=10^{-4}$ and with several different sound speeds. 
One can clearly see the two density channels. The process of clearing two density channels can be understood with three-body simulations (see §12).

One question always asked is about the rate of clearing the gap. Is a low mass planet able to open a gap or does it require a large planet mass? It depends not only on planet mass but also on sound speed. Fig. 4.5 plots the evolution with time of the density minima in the gap for several sound speeds and the planet mass $\mu=10^{-4}$. We see that for low sound speeds, the density in the gap is reduced much faster as for high sound speed runs. It also appears that the rate of density clearing is leveling off. This would indicate the disk is approaching a steady state. However, it remains unclear since the simulations did not go that far. In Fig. 4.6 the density profile is plotted for different planet masses but constant sound speed $c_{s}=0.05$. The time evolution of the minimum density in the channels is shown in Fig. 4.7.

The density profile is "W" shaped in radius. The reason is that the excited waves are non-linear near the planet and dissipate through shocks. In the region exterior to the protoplanet $\Delta r>r_{H}$, the waves deposit a positive angular momentum in the disk. The interior region $\Delta r<-r_{H}$ looses angular momentum. Hence, the disk material moves away from the orbit of the protoplanet. At the location where the waves are most efficiently deposited, the angular momentum is lost or gained at the maximum rate. That location is not only a function of planet mass but also a function of the temperature, i.e. the sound speed, in the disk (see §7.1). 
Therefore, the density profile is "W" shaped and although a gap-like structure is developing for all runs, there is still considerable amount of gas in the horseshoe region $|\Delta r|<r_{H}$. In the corotating frame of the planet, the gas flows towards the planet at $\Delta r<0$, but then reverses direction before it comes close to the planet (see Fig. 12.4). It then continues on a higher orbit $\Delta r>0$ until it is caught up by the planet again. The material in the horseshoe region is less affected by the planet than the material in the so-called separatrix region $r_{H}<|\Delta r|<\sqrt{12} r_{H}$. Furthermore, a density enhancement is observed at the Lagrangian points $L_{4}$ and $L_{5}$ where the gas is most stable in tadpole like orbits (Fig. 12.1). 


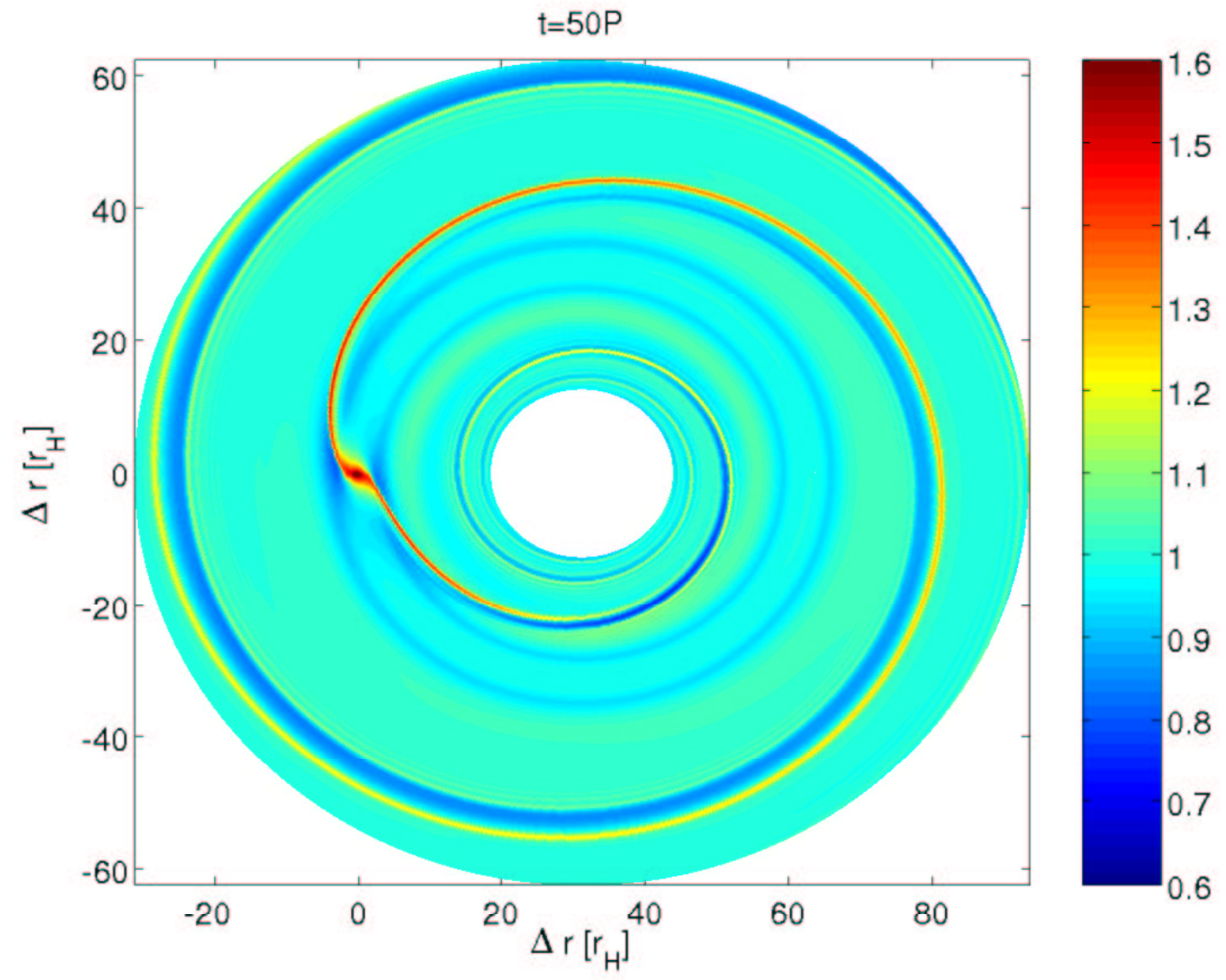

Figure 4.1: Snapshot of the disk surface density at $t=50 P$. See also Fig. 4.3 and 4.2 . 


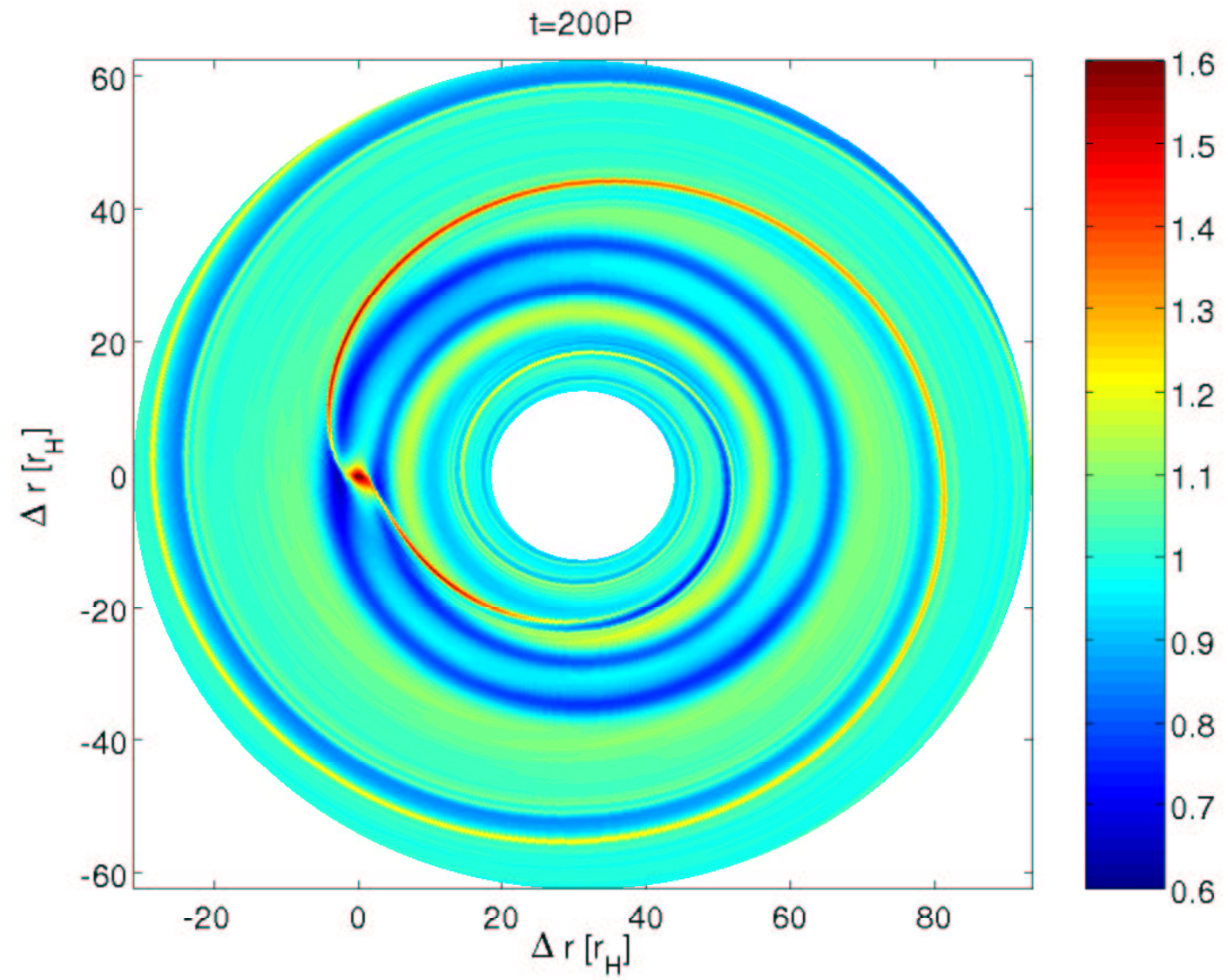

Figure 4.2: Snapshot of the disk surface density at $t=200 P$. See also Fig. 4.3 and 4.1 . 


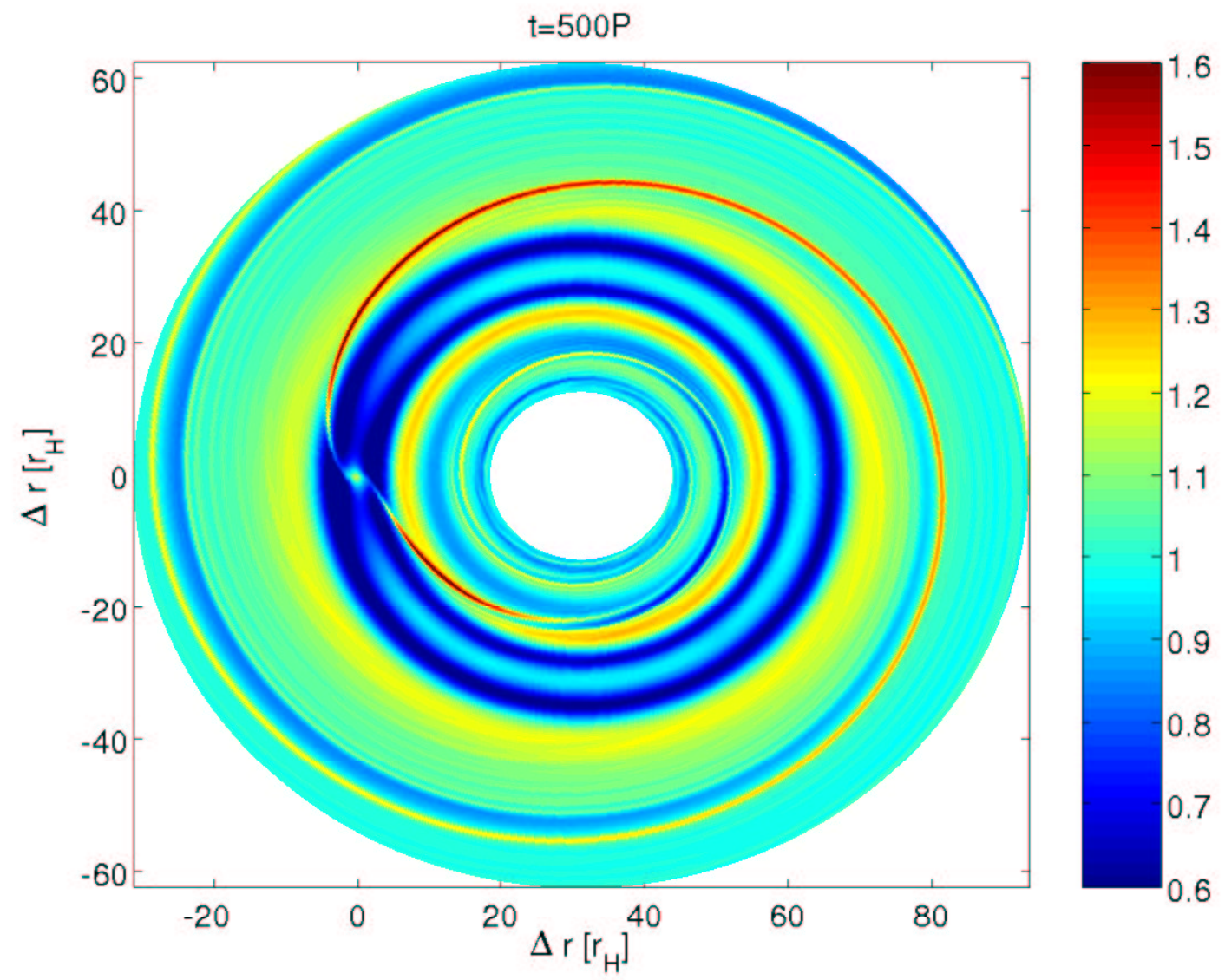

Figure 4.3: Snapshot of the disk surface density at $t=500 P$ for sound speed $c_{s}=0.05$ and planet mass $\mu=10^{-4}$. The tidal forces of the protoplanet start clearing out a gap with two density channels. Also, one can clearly see the filled Roche lobe of the planet and the two spiral arms emanating from the planet. 


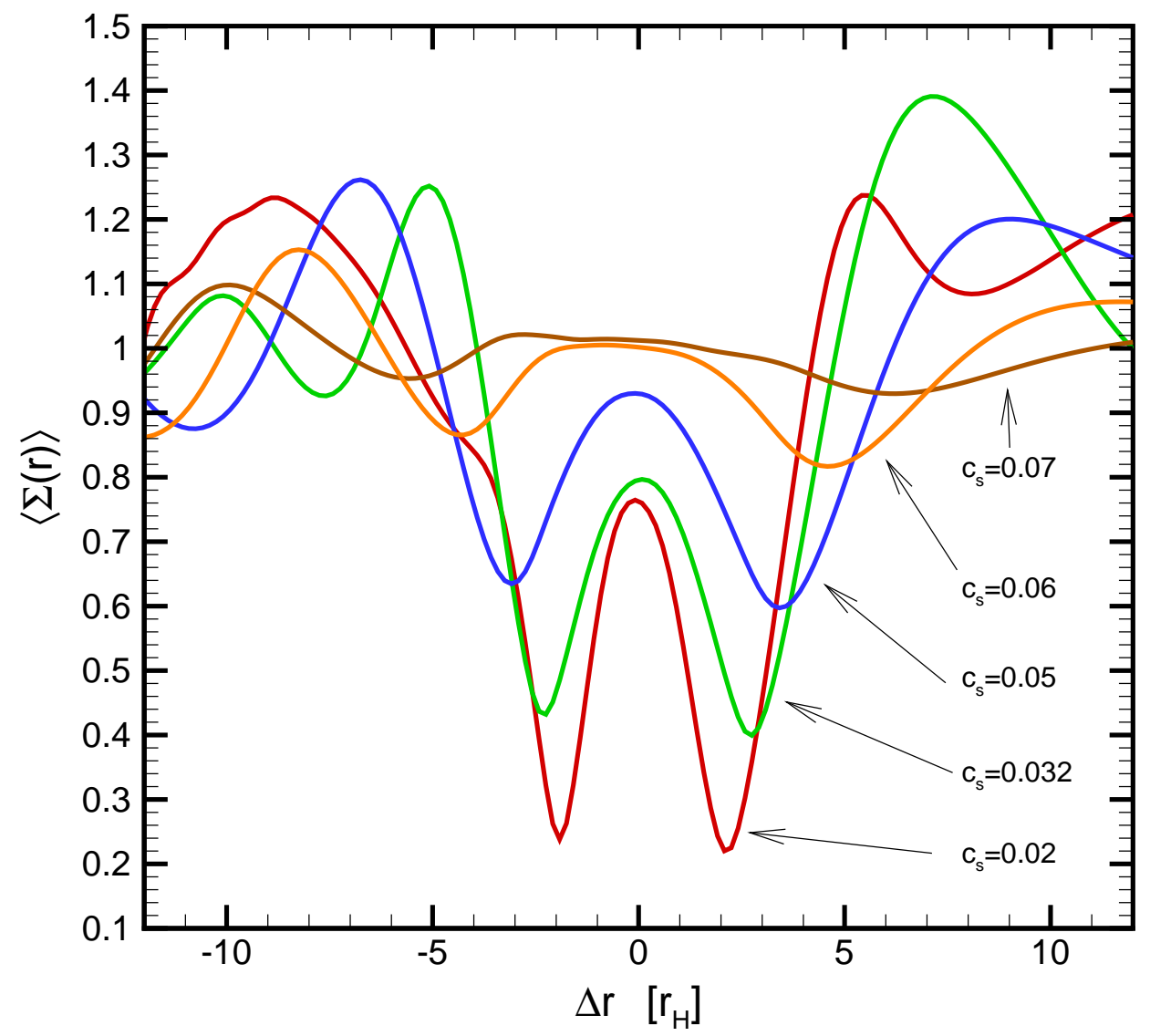

Figure 4.4: Density profile at a late time $t=500 P$ for several different sound speeds. The density is reduced in a "W"-type shape, i.e. two density minima channels form. The lower sound speed simulations show much deeper channels than the high sound speed runs. Also, note the shift in location of the minima for different sound speeds. 


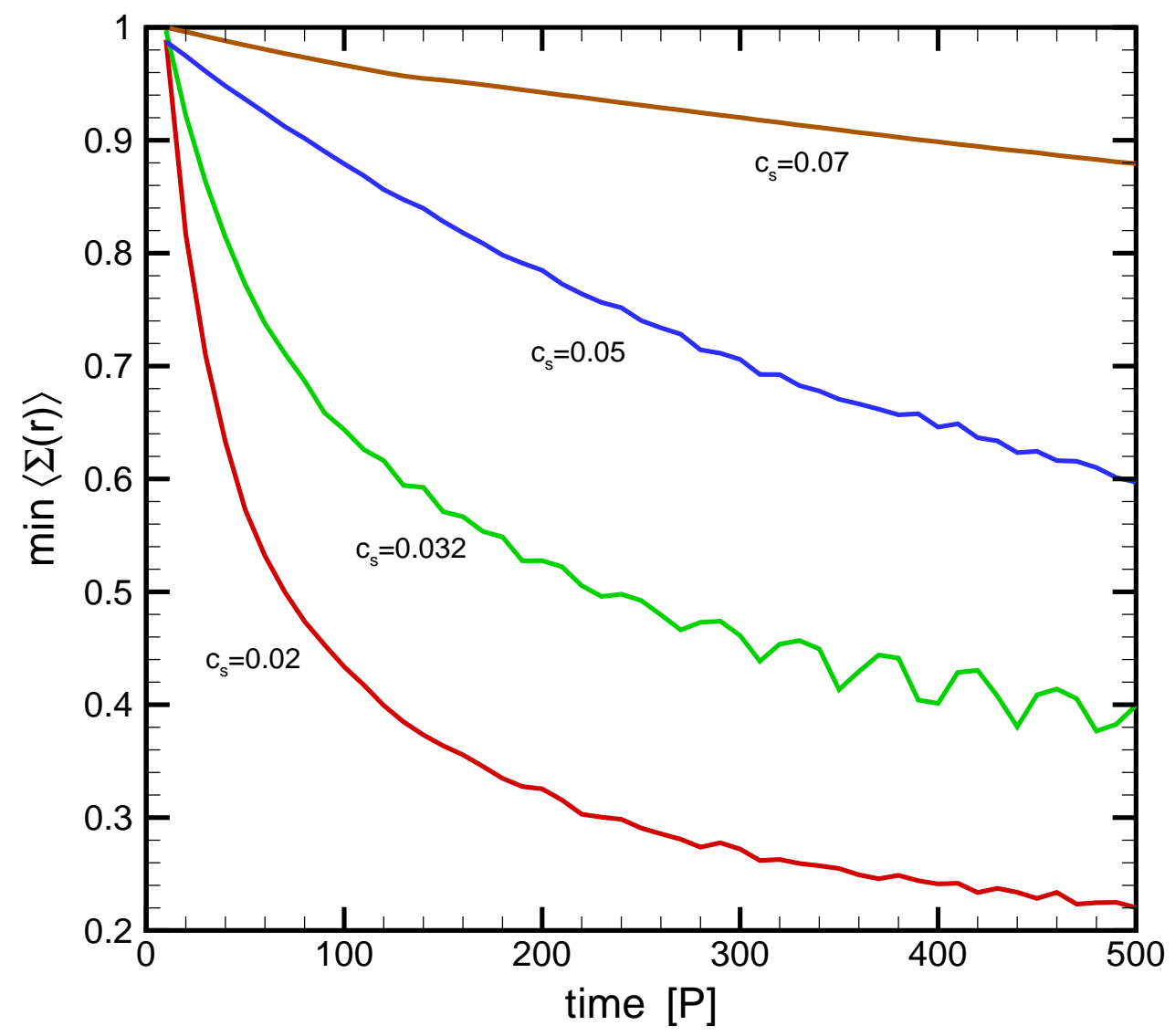

Figure 4.5: Density minimum evolution of the disk gap for several sound speeds. High sound speed runs show a much slower gap clearing rate than the lower sound speed runs. Also, it appears that the density reduction in the gap is leveling off. 


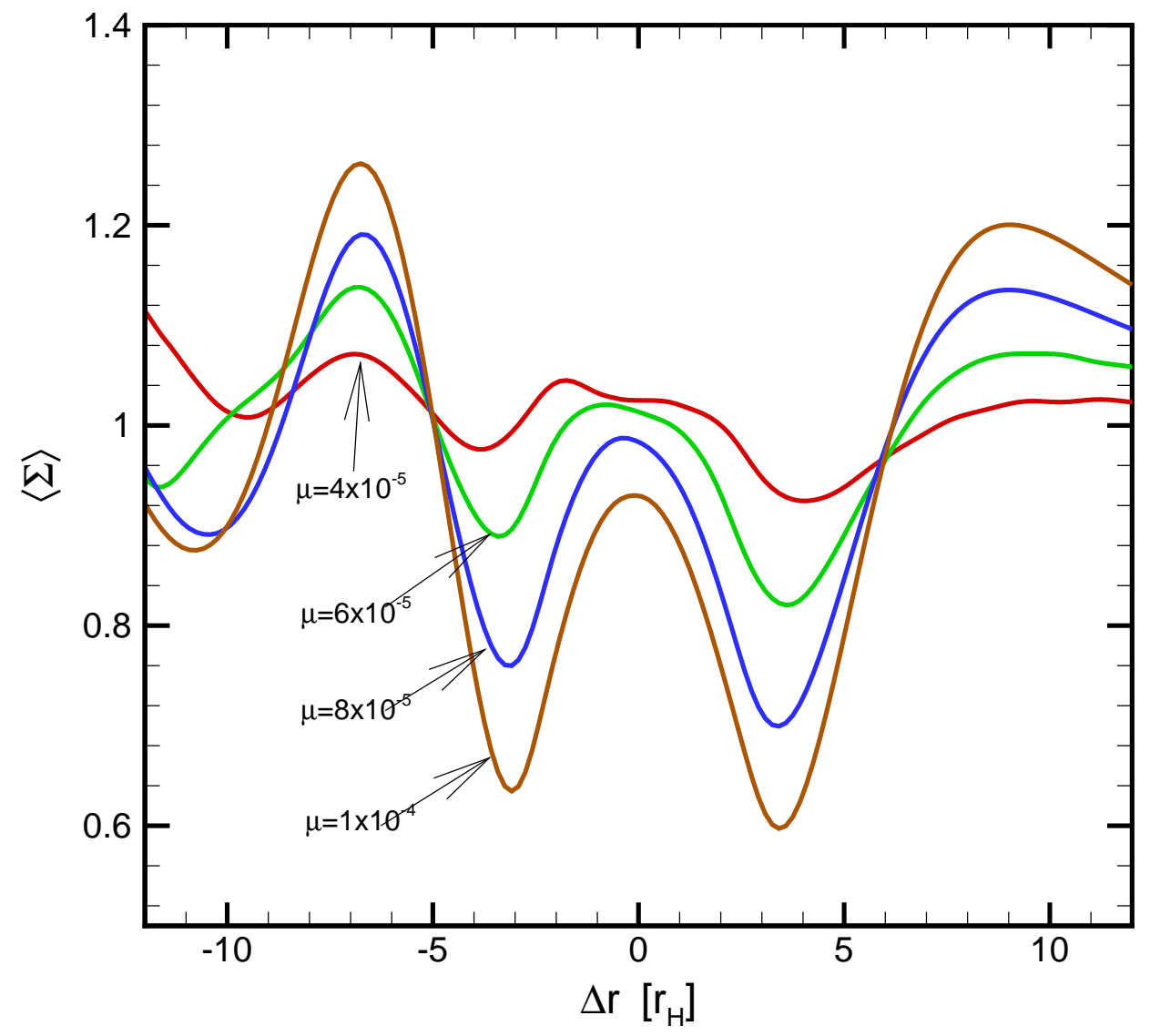

Figure 4.6: Density profile at a late time $t=500 P$ for several different planet masses $\mu$. The density is reduced in a "W"-type shape, i.e. two density minima channels form. The higher planet mass simulations show a much deeper channels than the lower planet mass runs. 


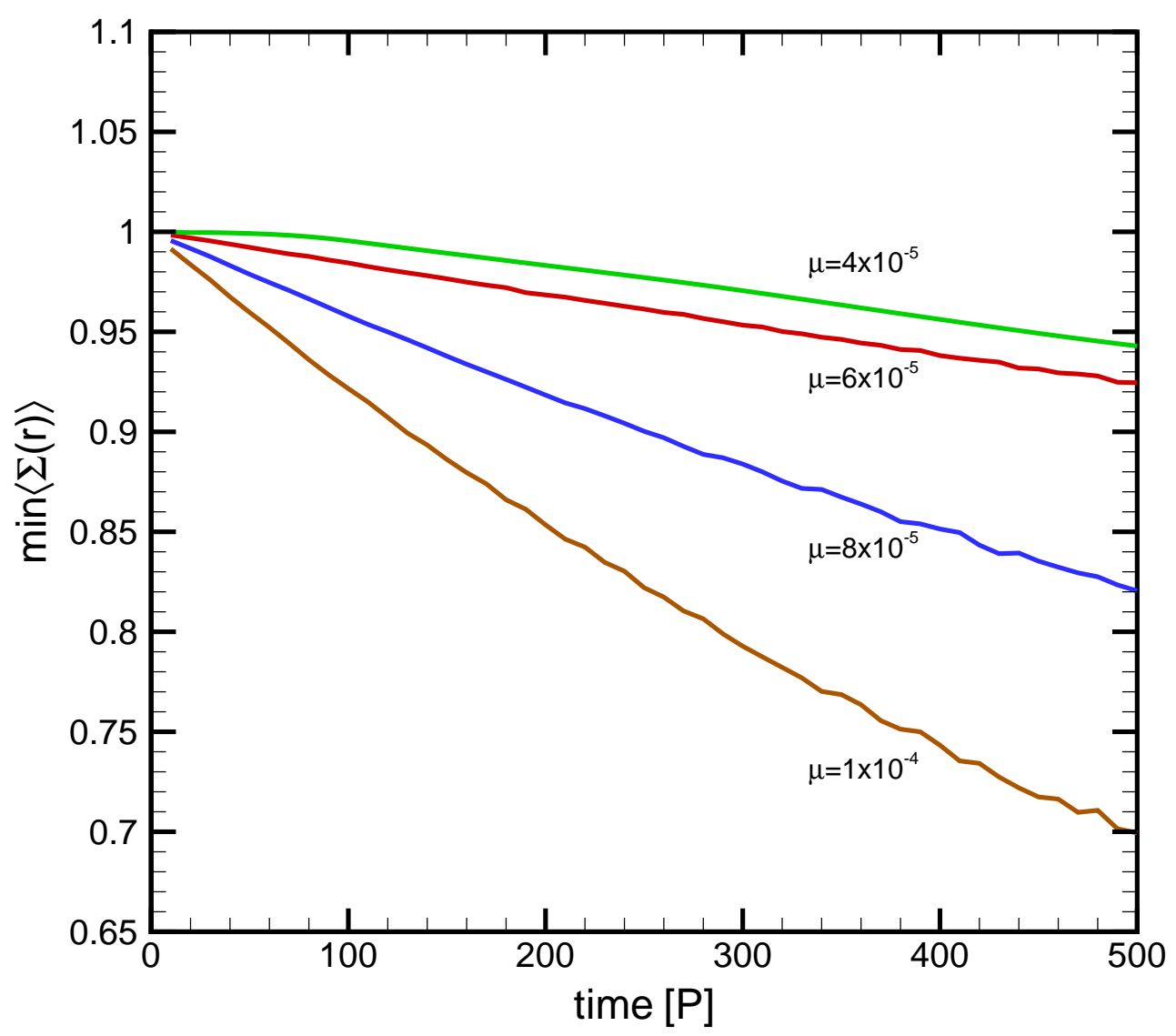

Figure 4.7: Density minimum evolution of the disk gap for several planet masses. High planet masses show a much faster gap clearing rate than the lower mass runs. 


\section{Chapter 5}

\section{Torque Evolution}

\subsection{Torque Equations}

A protoplanet embedded in a disk suffers gravitational torques, exerted by the surrounding material. If the density distribution were symmetric, with respect to the planet, the resulting total torque would be zero. Since the planet also interacts with the disk and changes its density distribution quite dramatically, the total torque will not be zero. The planet will respond to the disk torque by adjusting its semi-major axis, which leads to the migration phenomenon.

The torque exerted on the planet can be evaluated with the gravitational force $\boldsymbol{F}_{d}(r, \phi)$ from the disk element at $(r, \phi)$. The torque with respect to the star is then

$$
\boldsymbol{\tau}_{d}(r, \phi)=\left[\boldsymbol{r}_{p} \times \boldsymbol{F}_{d}(r, \phi)\right] \cdot \hat{z}
$$


where we are only interested in the $z$ component due to the two-dimensional nature of our disk. By calculating the cross product (see Fig. 5.1), we find

$$
\left|\boldsymbol{\tau}_{d}\right|(r, \phi)=\left|\boldsymbol{r}_{p}\right| \cdot\left|\boldsymbol{F}_{d}(r, \phi)\right| \sin \gamma=\frac{G M_{p} M_{g a s}}{d^{2}} \frac{r_{i j}}{d_{i j}} \sin \phi
$$

where $i, j$ refers to the grid cell. Finally by integrating over the whole disk we obtain the total disk torque in normalized $|\tilde{\tau}|=|\tau| / G M_{p}$ quantities

$$
T_{d}=\int \frac{r^{2}}{d^{3}} \sin \phi d r d \phi
$$

which is numerically done in the code as a sum over $i$ and $j$.

One sees that the largest contribution to the total torque will come from close to the planet since $T_{d} \propto d^{-3}$. If the region around the planet or even the whole co-orbital region is excluded from the torque integration, the result will differ dramatically (see $\S 5.2$ ).

Planet and disk interact mutually. If the disk is changing due to the gravitational force of the planet, the planet orbit will change due to the disk force. If we consider a planet moving on a circular orbit, we find that the rate of change of its semi-major axis $r_{p}$, caused by en external torque $T_{d}$, is to first order

$$
\frac{d r_{p}}{d t}=\frac{2 T_{d}}{M_{p} r_{p} \Omega_{p}}
$$




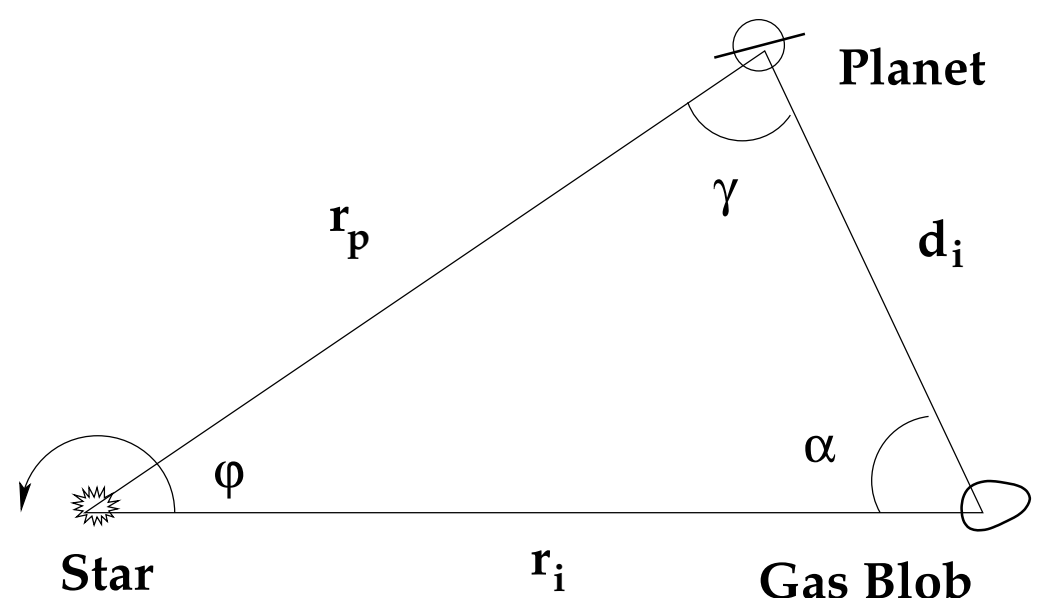

Figure 5.1: Diagram of the disk with a planet used for torque calculations.

where $\Omega_{p}$ is the angular velocity of the planet (D'Angelo et al., 2002; Ward, 1997).

\subsection{Phase Transition}

Our simulation results are generally in agreement with previous simulations for the planet on a fixed circular orbit (see Masset, 2002). The generic structures, produced in the disk by the tidal interactions between the planet and its surrounding flows, are the same. Since the planet mass is relatively small, only density dips are generated with no gaps (at least during the few hundred orbits simulated here).

One of the key finding is shown in Fig. 5.2 which displays the total torque on the planet from the disk. It has two very distinct phases:

(1) Phase I: the total torque is negative and smooth, modulated by a period that is similar to the libration period near the $L_{4}$ and $L_{5}$ points $\left(\tau_{l i b} \sim \sqrt{4 / 27 \mu} \sim 40 P\right.$ where $P$ is one orbit at $r=1$ ). 
(2) Phase II: the total torque shows very large amplitude and fast oscillations with a quasi-period of a few orbits. We see such a behavior in all simulations with intermediate sound speeds. The time when the "phase" transition occurs is different though. It starts at $t \approx 40 P, 90 P$, and $210 P$ for $c_{s}=0.04,0.05$ and 0.06 , respectively.

To show that the torque is due to features in the co-orbital region, Fig. 5.3 describes the contribution from several regions. The inner disk $r<0.9$ and the outer disk $r>1.1$ shows no phase transition. Instead both torques are very smooth. Only in phase III (see §5.3), there is some oscillation from both regions. On the other hand, the torque from the co-orbital region $-3 r_{H}<\Delta r<3 r_{H}$ is very different. It shows the same features as in Fig. 5.2. This shows the torque behavior is due to the co-orbital region. The simulation in Fig. 5.3 has a sound speed $c_{s}=0.04$. As we see later, vortices are responsible for that phase transition. They form for that particular sound speed at $\Delta r \approx \pm 2 r_{H}$. If that co-orbital region would be excluded from the torque calculation, as has been done for many papers before (e.g. D'Angelo et al., 2002, and referenced therein), the result would be vastly different.

\subsection{Phase III Transition}

If we follow the torque evolution long enough, we even find a third phase. As we will see later, secondary instabilities have developed vortices in the co-orbital 


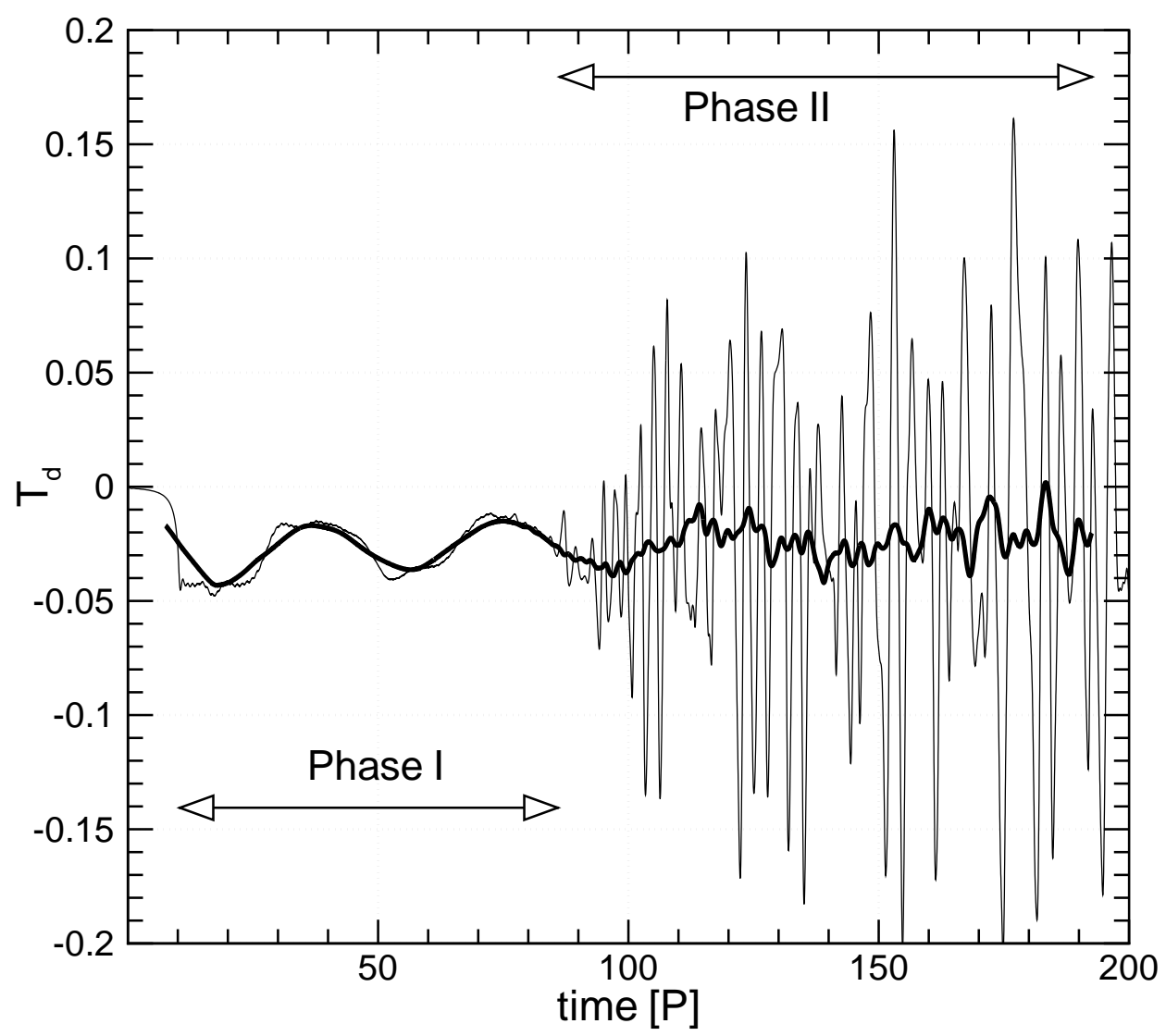

Figure 5.2: Time evolution of the total torque $T_{d}$ exerted on the planet. Phase I $(t<90 P)$ : The total torque is negative, smooth, and shows an oscillation period of $\approx 40 P$ which is consistent with the libration period. We used a turn-on time of 10 orbits for the planet mass. Phase II $(t>90 P)$ : The torque starts to show oscillations with increasingly high amplitudes. During the transition from Phase I to Phase II, vortices emerge around $\Delta r \approx 2.4 r_{H}$. These vortices experience closeencounters with the planet, resulting in large amplitude variations in the torque. The bold curve depicts a moving average of $T_{d}$ with a window of $t=15 P$ orbits. 


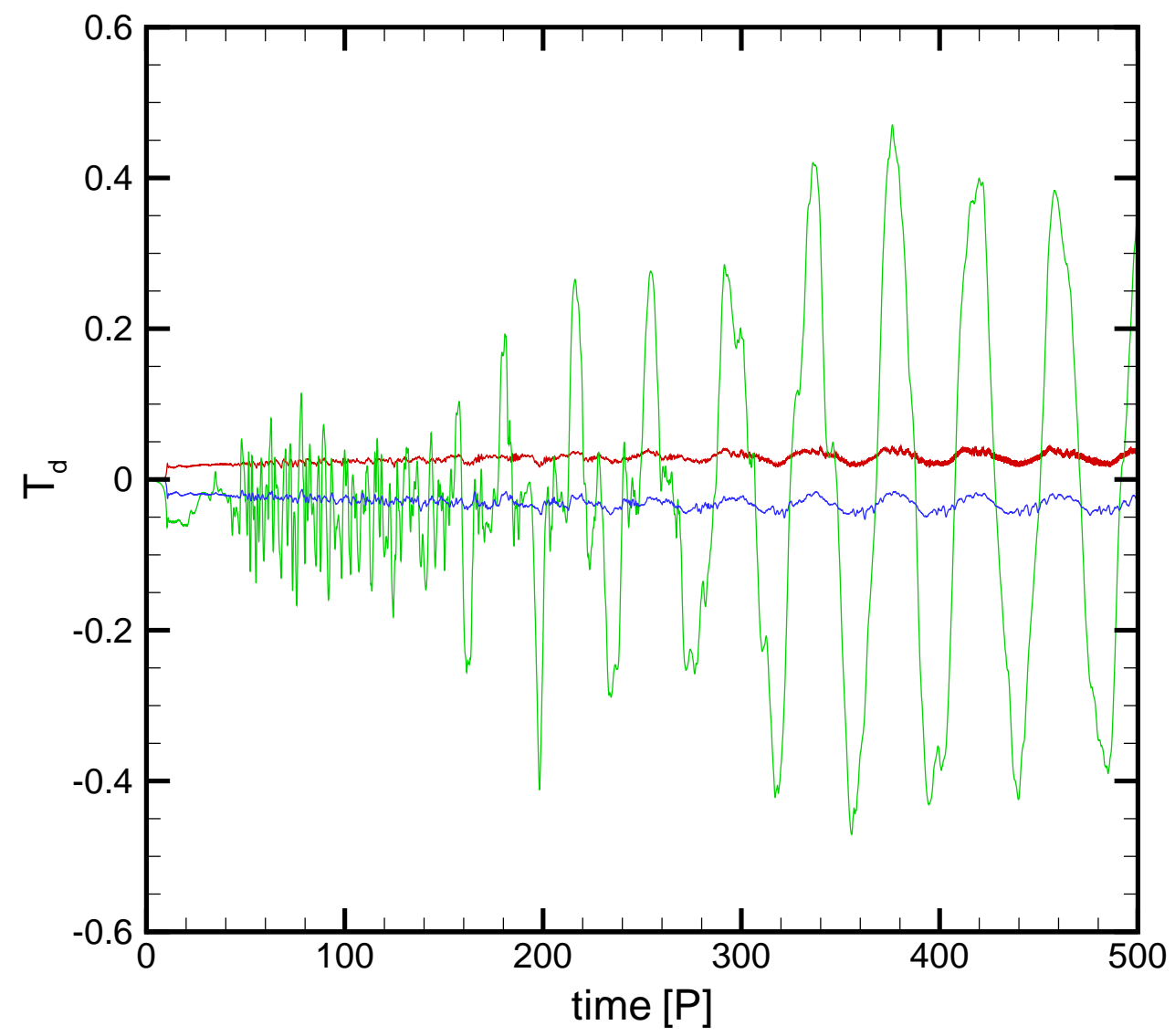

Figure 5.3: Torque from different regions in the disk. The contribution from the inner (red) disk and the outer (blue) disk do not show a phase transition. Only the co-orbital region (green) generates vortices and, hence, the torque depicts a phase transition. 
region. They are having frequent close encounters with the planet. Each encounter slows down the vortex experiencing the pressure gradient at the spiral shock. This short delay leaves time for the vortex following behind to catch up and eventually merge with the first vortex. Previously Li et al. (2001) observed that vortices with a small but non-zero relative velocity are able to merge and form a single supervortex. The situation here is not so different. Fig. 5.4 illustrates the potential vorticity for a $c_{s}=0.04$ simulation at $t=110 P$ and $t=400 P$. At the later time, Fig. 5.5 shows that the torque develops much stronger amplitudes and a longer period, hereafter called phase III. The disk is at $t=110 P$ and $t=400 P$ in phase II and phase III respectively. In phase II the potential vorticity profile starts several small vortices at $|\Delta r| \approx 2 r_{H}$ whereas in phase III at $t=400 P$ all single vortices have merged into a large vortex orbiting in the co-orbital region. At that time they exert torques on the planet with a lower frequency but a stronger amplitude. 


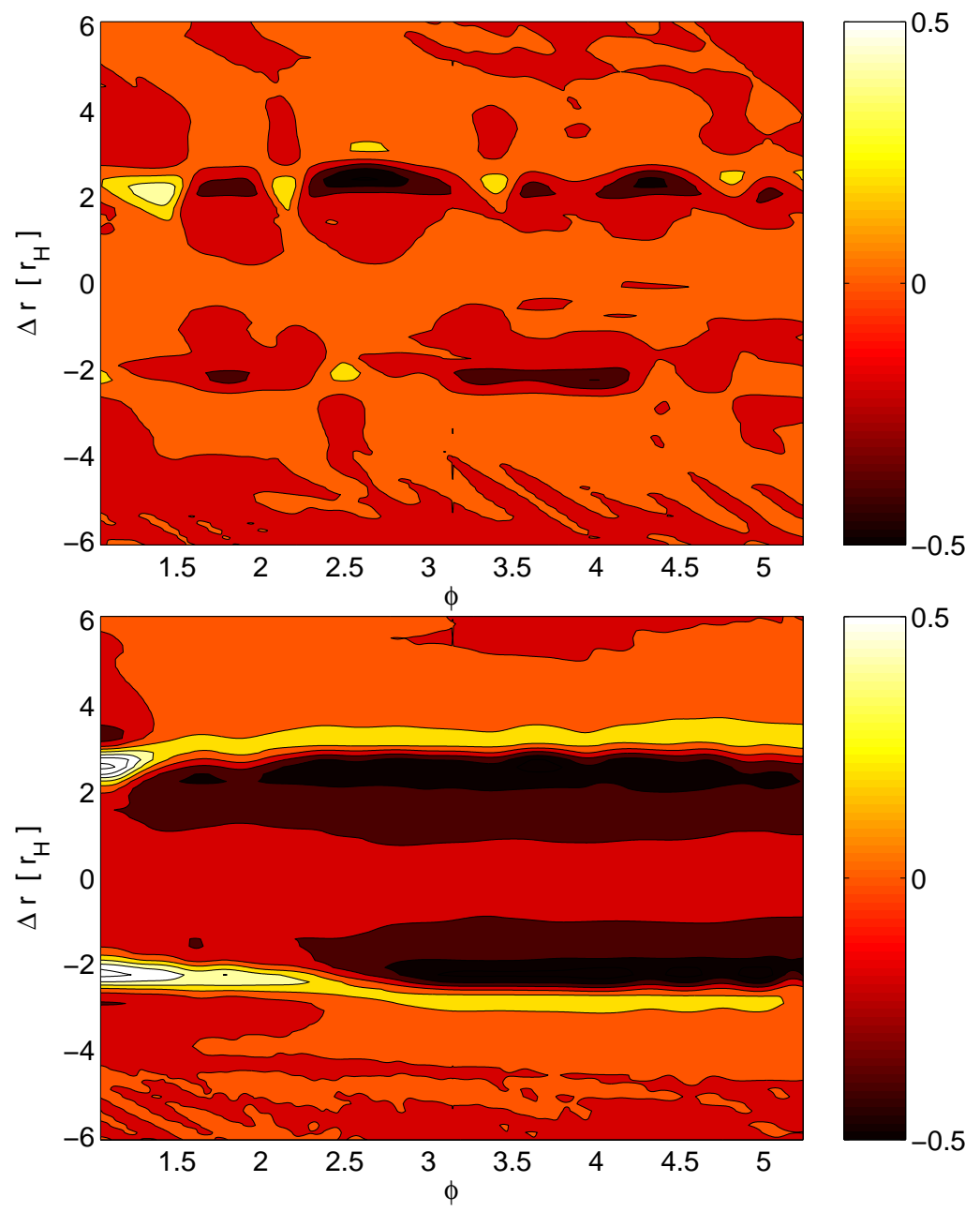

Figure 5.4: Potential vorticity image for $c_{s}=0.04$ at $t=110 P$ (upper) and at $t=$ $400 P$ (lower). An azimuthally averaged potential vorticity profile was subtracted to pronounce the potential vorticity features. At the earlier stage at $t=110 P$ there are several small vortices at $|\Delta r| \approx 2 r_{H}$. At a later stage those vortices have merged and the PV image (with the same azimuthally averaged subtraction) does not show anymore those single vortices. Instead they have merged into a large blob revolving in the horseshoe region exerting strong torques onto the planet. 


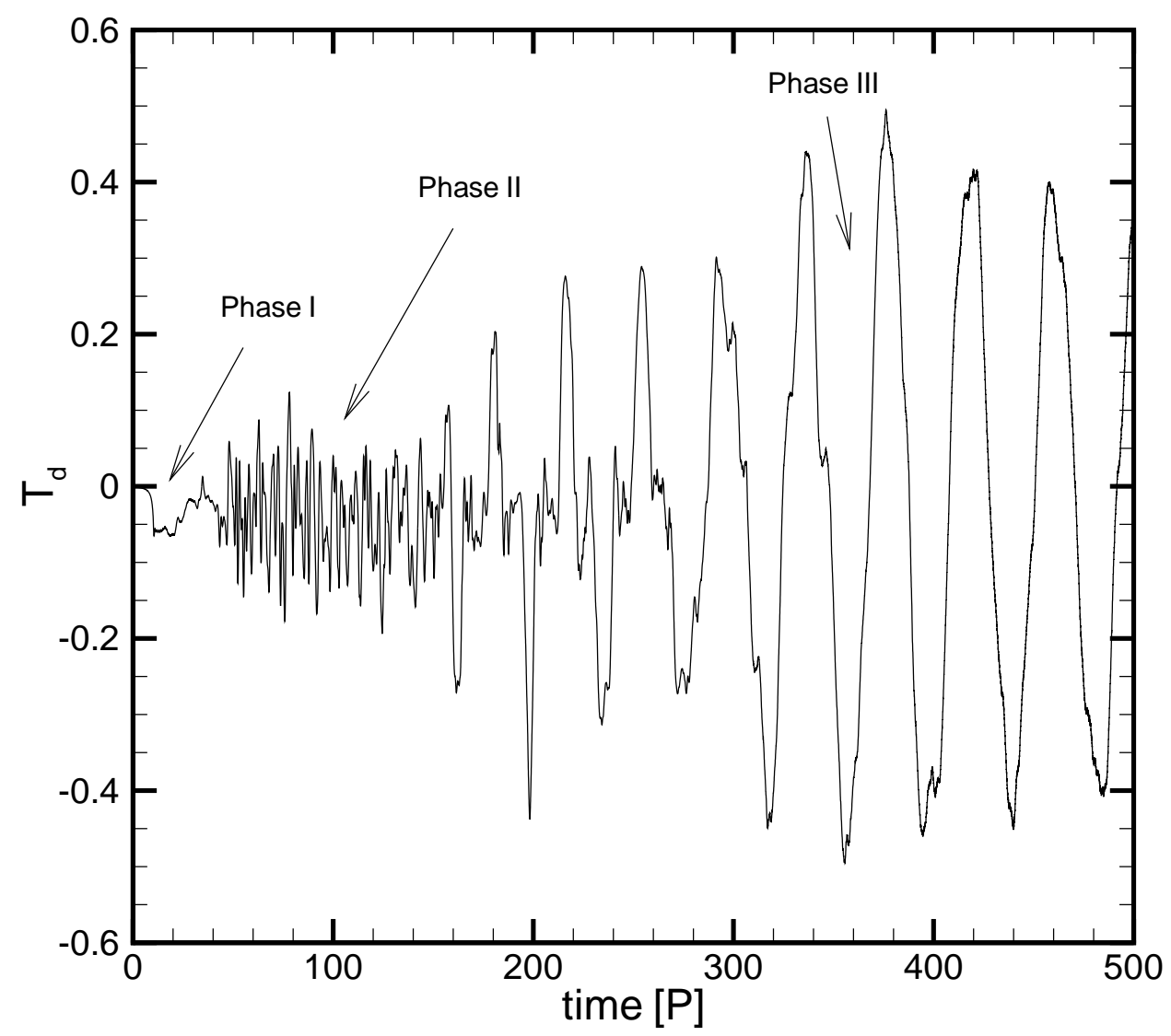

Figure 5.5: Long term torque evolution of a simulation with sound speed $c_{s}=0.04$. Lower sound speed runs show an additional transition into phase III. Merging vortices increase the total torque and also increase the oscillation period because fewer but bigger vortices are passing by the planet. 


\section{Chapter 6}

\section{PV, Shocks, and Instabilities}

\subsection{Vorticity and Vortensity}

Although the Euler equations (2.9 and 2.10), together with an equation of state, are a full set of equation, it makes sometimes sense to study the evolution of a hydrodynamic system with a derived dynamic variable. One derived dynamic variable of preeminent importance in geophysical fluid dynamics is the vorticity vector $\boldsymbol{\omega}$, defined as the curl of the velocity field, i.e.,

$$
\boldsymbol{\omega}=\nabla \times \boldsymbol{v}
$$

Another, even more important variable in geophysical flows is potential vortic- 
ity (PV or vortensity), which is the vorticity divided by the density

$$
\boldsymbol{\zeta}=\frac{\nabla \times \boldsymbol{v}}{\rho}
$$

As we will see later, potential vorticity is such an important parameter because it is conserved along streamlines in dissipation-less flow. In our case, and in the case of geophysical flows, the fluids are undergoing a global rotation $\boldsymbol{\Omega}$. It is sometimes advantageous to study them in a corotating frame of reference. The vorticity of the fluid as observed from an inertial, non-rotating frame is called absolute vorticity, $\boldsymbol{\omega}_{a}$, and it is simply defined as the curl of the velocity observed in the non-rotating frame, i.e.,

$$
\boldsymbol{\omega}_{a}=\nabla \times(\tilde{\boldsymbol{v}}+\boldsymbol{\Omega} \times \boldsymbol{r})=\boldsymbol{\omega}+2 \boldsymbol{\Omega}
$$

where $\boldsymbol{\omega}$ is the relative vorticity and $\tilde{\boldsymbol{v}}$ is the relative velocity in the rotating frame of reference. In that frame it then becomes

$$
\omega=(\nabla \times \tilde{\boldsymbol{v}}) \cdot \hat{z}=\frac{1}{r} v_{\phi}+\frac{\partial v_{\phi}}{\partial r}-\frac{1}{r} \frac{\partial v_{r}}{\partial \phi}-2 \Omega
$$

with $\Omega$ as the angular velocity of the rotating frame of reference.

To further examine the nature of vorticity, we will now derive directly an equation for the relative vorticity vector. 
The momentum equation with $\tilde{\boldsymbol{v}}$ as seen in the rotating frame of reference

$$
\frac{\partial \tilde{\boldsymbol{v}}}{\partial t}-\boldsymbol{\Omega} \times(\boldsymbol{\Omega} \times \boldsymbol{r})+2 \boldsymbol{\Omega} \times \tilde{\boldsymbol{v}}=-\frac{1}{\Sigma} \nabla P+\nabla \Phi
$$

can be rewritten using the vector identity

$$
\boldsymbol{\omega} \times \tilde{\boldsymbol{v}}=\nabla \times \tilde{\boldsymbol{v}} \times \tilde{\boldsymbol{v}}=(\tilde{\boldsymbol{v}} \cdot \nabla) \tilde{\boldsymbol{v}}-\frac{1}{2} \nabla|\tilde{\boldsymbol{v}}|^{2}
$$

into

$$
\frac{\partial \tilde{\boldsymbol{v}}}{\partial t}+(2 \boldsymbol{\Omega}+\boldsymbol{\omega}) \times \tilde{\boldsymbol{v}}=-\frac{\nabla P}{\Sigma}+\nabla\left(\Phi-\frac{|\tilde{\boldsymbol{v}}|^{2}}{2}\right)
$$

To find an equation for $\boldsymbol{\omega}$, we now take the curl of Eq. (6.7) and obtain

$$
\frac{\partial \boldsymbol{\omega}}{\partial t}+\nabla \times[(2 \boldsymbol{\Omega}+\boldsymbol{\omega}) \times \tilde{\boldsymbol{v}}]=\frac{\nabla \Sigma \times \nabla P}{\Sigma^{2}}
$$

since the forces in our system are conservative, they drop out with taking the curl of a gradient $\nabla \times(\nabla \Phi)=0$.

Now for any two vectors $\boldsymbol{A}$ and $\boldsymbol{B}$ (Weisstein, 2004),

$$
\nabla \times(\boldsymbol{A} \times \boldsymbol{B})=\boldsymbol{A}(\nabla \cdot \boldsymbol{B})+(\boldsymbol{B} \cdot \nabla) \boldsymbol{A}-\boldsymbol{B}(\nabla \cdot \boldsymbol{A})-(\boldsymbol{A} \cdot \nabla) \boldsymbol{B},
$$

so that

$$
\nabla \times\left(\boldsymbol{\omega}_{a} \times \tilde{\boldsymbol{v}}\right)=\boldsymbol{\omega}_{a}(\nabla \cdot \tilde{\boldsymbol{v}})+(\tilde{\boldsymbol{v}} \cdot \nabla) \boldsymbol{\omega}_{a}-\left(\boldsymbol{\omega}_{a} \cdot \nabla\right) \tilde{\boldsymbol{v}},
$$


since $\boldsymbol{\omega}_{a}=\boldsymbol{\omega}+2 \boldsymbol{\Omega}$ has zero divergence. Hence Eq. (6.8) becomes

$$
\frac{\partial \boldsymbol{\omega}}{\partial t}+(\tilde{\boldsymbol{v}} \cdot \nabla) \boldsymbol{\omega}_{a}=\left(\boldsymbol{\omega}_{a} \cdot \nabla\right) \tilde{\boldsymbol{v}}-\boldsymbol{\omega}_{a}(\nabla \cdot \tilde{\boldsymbol{v}})+\frac{\nabla \Sigma \times \nabla P}{\Sigma^{2}}
$$

The $(\nabla \Sigma \times \nabla P) / \Sigma^{2}$ term in equation $(6.11)$ is called the baroclinic term. Using an isothermal equation of state with $P=P(\Sigma)$, i.e. a barotropic relation, the baroclinic term in Eq. (6.11) will vanish.

Eq. (6.11) will then read as

$$
\frac{\mathrm{D} \boldsymbol{\omega}_{a}}{\mathrm{D} t}=\left(\boldsymbol{\omega}_{a} \cdot \nabla\right) \tilde{\boldsymbol{v}}-\boldsymbol{\omega}_{a}(\nabla \cdot \tilde{\boldsymbol{v}})
$$

with $D / D t=\partial / \partial t+\tilde{\boldsymbol{v}} \cdot \nabla$. Since $\Omega$ is a constant, $D \boldsymbol{\omega}_{a} / D t=D \boldsymbol{\omega} / D t$. In two-dimensional coordinates, the first term in Eq. $(6.12)\left(\boldsymbol{\omega}_{a} \cdot \nabla\right) \tilde{\boldsymbol{v}}=0$.

Next, we derive potential vorticity which we will obtain by multiplying Eq. (6.12) with $1 / \Sigma$. By substituting the continuity equation

$$
\nabla \cdot \tilde{\boldsymbol{v}}=-\frac{1}{\Sigma} \frac{\mathrm{D} \Sigma}{\mathrm{D} t}
$$

this yields the conservation law of potential vorticity

$$
\frac{\mathrm{D}}{\mathrm{D} t}\left(\frac{2 \Omega+\omega}{\Sigma}\right)=0
$$


for two-dimensional geometries, isothermal equation of state, and dissipation-less flow. That means that a fluid element will always keep the same potential vorticity value except when it experiences dissipation, i.e. it passes through a shock or a viscous boundary layer.

\subsection{PV Evolution and Secondary Instabilities}

Now we want to discuss the obvious question arising from $§ 5.2$ : What causes such large amplitude and fast changes in torques? Closer inspection of the coorbital region at times around $90 P$ (using $c_{s}=0.05$ as an example) reveals that vortices are generated in the separatrix region. This development is shown in Fig. 6.2-6.4, which depict maps of potential vorticity $\zeta$ through the times when the system undergoes its "phase" transition. One can see that these vortices are anti-cyclones which are also blobs of higher densities (not shown here). Being in the separatrix region, they experience very close encounters with the planet repeatedly, thus exerting strong "impulses" to the planet. This results in the large amplitude and fast variations in the torque evolution.

To understand this phenomenon further, in Fig. 6.1, the azimuthally averaged radial PVprofile $\langle\zeta\rangle$ for $c_{s}=0.05$ is plotted at different times $t=0,20 P, 40 P, 80 P$, and $t=120 P$. One sees that this profile deviates progressively away from the initially flat profile, developing two prominent structures at $|\Delta r| \approx 2-3 r_{H}$, with a minimum at $\Delta r \approx 2.4 r_{H}$ and a maximum at $\Delta r=3.4 r_{H}$. Comparing with 
Fig. 6.4, vortices apparently emerge from the minima.

The existence of the extrema (or inflection points) in the potential vorticity profile is usually regarded as a necessary condition for non-axisymmetric instabilities in rotating shear flows (Drazin \& Reid 1981). The progressive steepening of this profile, say, at $\Delta r \approx 2.4 r_{H}$ until $t<90 P$ and the subsequent flattening (see the curve at $t=120 P$ ) strongly suggest that there is a secondary instability which is excited around $\Delta r \approx 2.4 r_{H}$ and vortices are the non-linear outcome of such an instability.

How could the potential vorticity profile develop such inflection points and structures from an initially flat and stable profile? As can be seen from the evolution equation for potential vorticity

$$
D(\zeta \hat{z}) / D t=(\zeta \hat{z} \cdot \nabla) \mathbf{v}=0 \text { in } 2 \mathrm{D},
$$

where $D / D t$ is the Lagrangian derivative, that $\zeta$ is conserved along the streamlines in 2D (Korycansky \& Papaloizou 1996). Thus, the initial uniform $\zeta$ distribution should be preserved in an inviscid disk. This conclusion, however, is only true provided that streamlines can be defined everywhere in a dissipation-less flow.

Disk flows in the co-orbital region around the planet, however, do not satisfy this condition (except perhaps for flows within $|\Delta r|<r_{H}$ ) because of the two sets of spiral shocks produced by the planet. These two sets of shocks emanate 
from close to the planet and cut through the whole disk. Flow lines (Fig. 6.5) which encounter these shocks are broken and the dissipation in shocks breaks the potential vorticity conservation. The consequence of the shock is equivalent to that of a strong viscous stress in the momentum equation, the curl of which, does not vanish.

Fig. 6.6 shows the starting location and the perpendicular Mach number $M_{\perp}$ of the inner shock for $c_{s}=0.05$ There is no shock between the planet and $\Delta r \approx$ $1.1 r_{H}$. The shock remains weak after that with a maximum of about $M_{\perp} \approx 1.3$. To establish the role of shocks in breaking the potential vorticity conservation, three runs with different sound speeds $c_{s}=0.04,0.05,0.06$ were performed. Even though they only span a small range, the effects are quite clear. In Fig. 6.7, the azimuthally averaged PV profile is plotted for these three runs at a time just before vortices emerge. Vortices first appear at the minimum of the "valley" in the profiles which moves outwards as $c_{s}$ increases. This growth pattern is consistent with the fact that the starting location of the shocks is also moving away from the planet when the sound speed increases.

Note that the cause for emerging and growing inflection points in the azimuthally averaged $\zeta$ distribution presented here is different from that due to its re-arrangement along the horseshoe orbits (Balmforth and Korycansky 2001). A calculation with a more general initial $\Sigma$ (and $\zeta$ ) distribution confirms that the onset of the shearing instability occurs at an earlier epoch. The appearance of 
the subsequently self-excited vortices is quite similar to the development of the Kelvin-Helmholtz (KH) instability observed at shear interfaces.

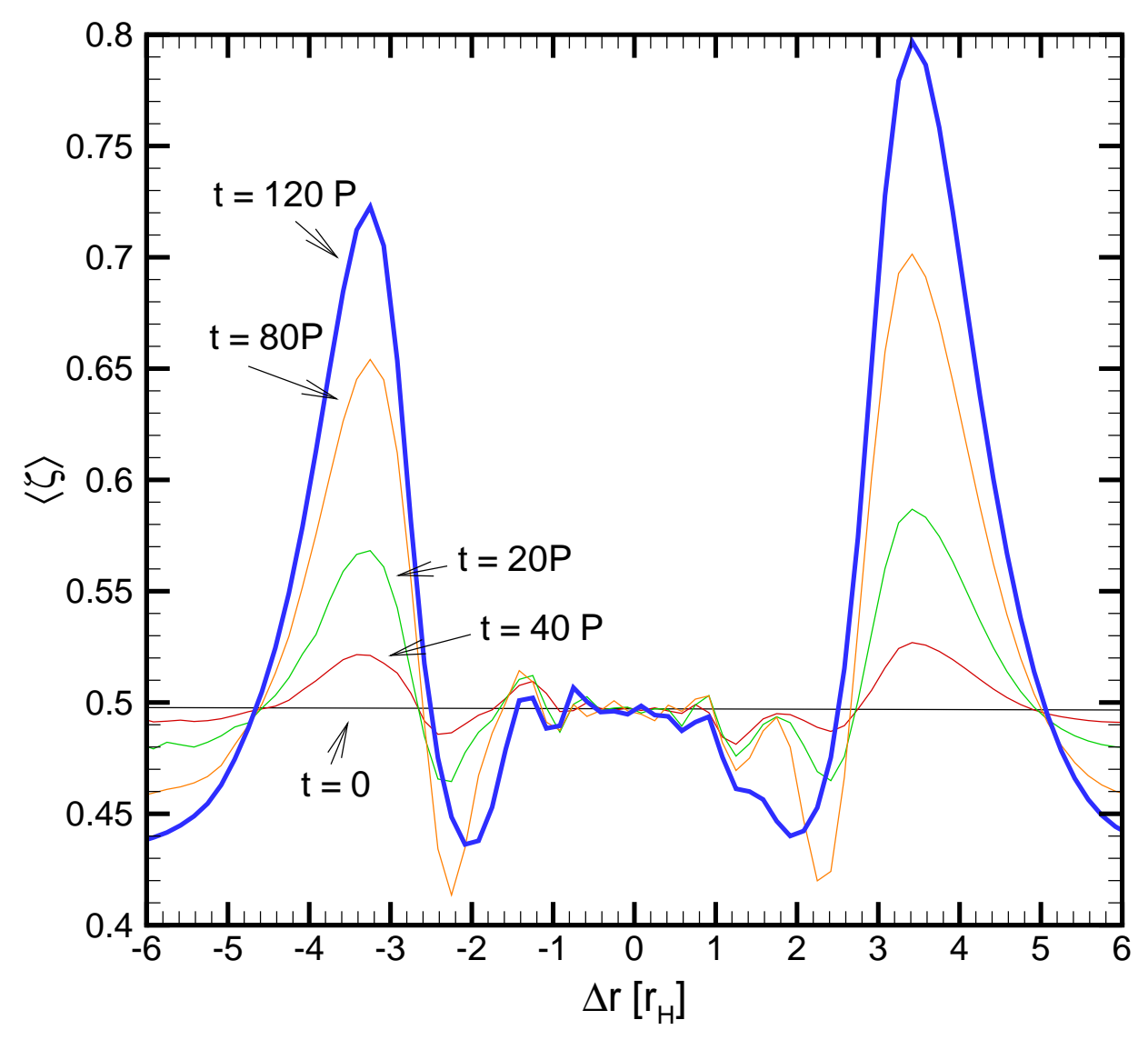

Figure 6.1: Evolution of the azimuthally averaged potential vorticity $\langle\zeta\rangle$ for $c_{s}=$ 0.05 at different times $t=0,20 P, 40 P, 80 P, 120 P$. Take the structure at $\Delta r \sim$ $2-3 r_{H}$ as an example. The profile develops dips $\left(\Delta r=2.4 r_{H}\right)$ and peaks $(\Delta r=$ $\left.3.4 r_{H}\right)$ progressively from $t=0-80 P$ until the vortices are produced at $t=90 P$, after which the minimum flattens (see the bold $t=120 P$ curve). 


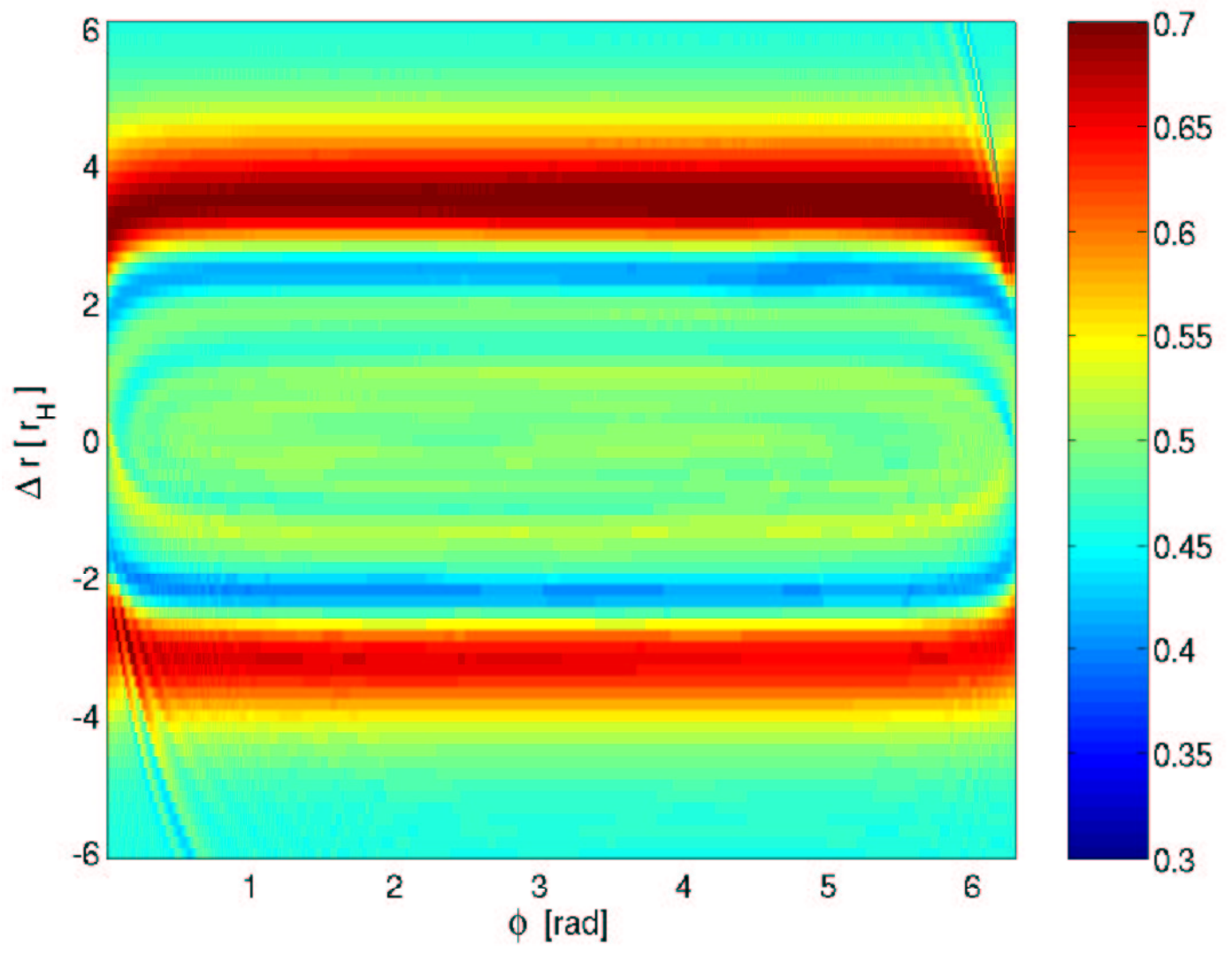

Figure 6.2: Potential vorticity $\zeta$ showing the production of vortices. The planet is located at $(\Delta r, \phi)=(0,0)$ and the radial distance to the planet is given in units of the Hill (Roche) radius $r_{H}$. Here, $c_{s}=0.05$. At $t=80 P$ the potential vorticity profile is relatively smooth, with two "channels" at roughly $|\Delta r| \approx 2-3 r_{H}$. 


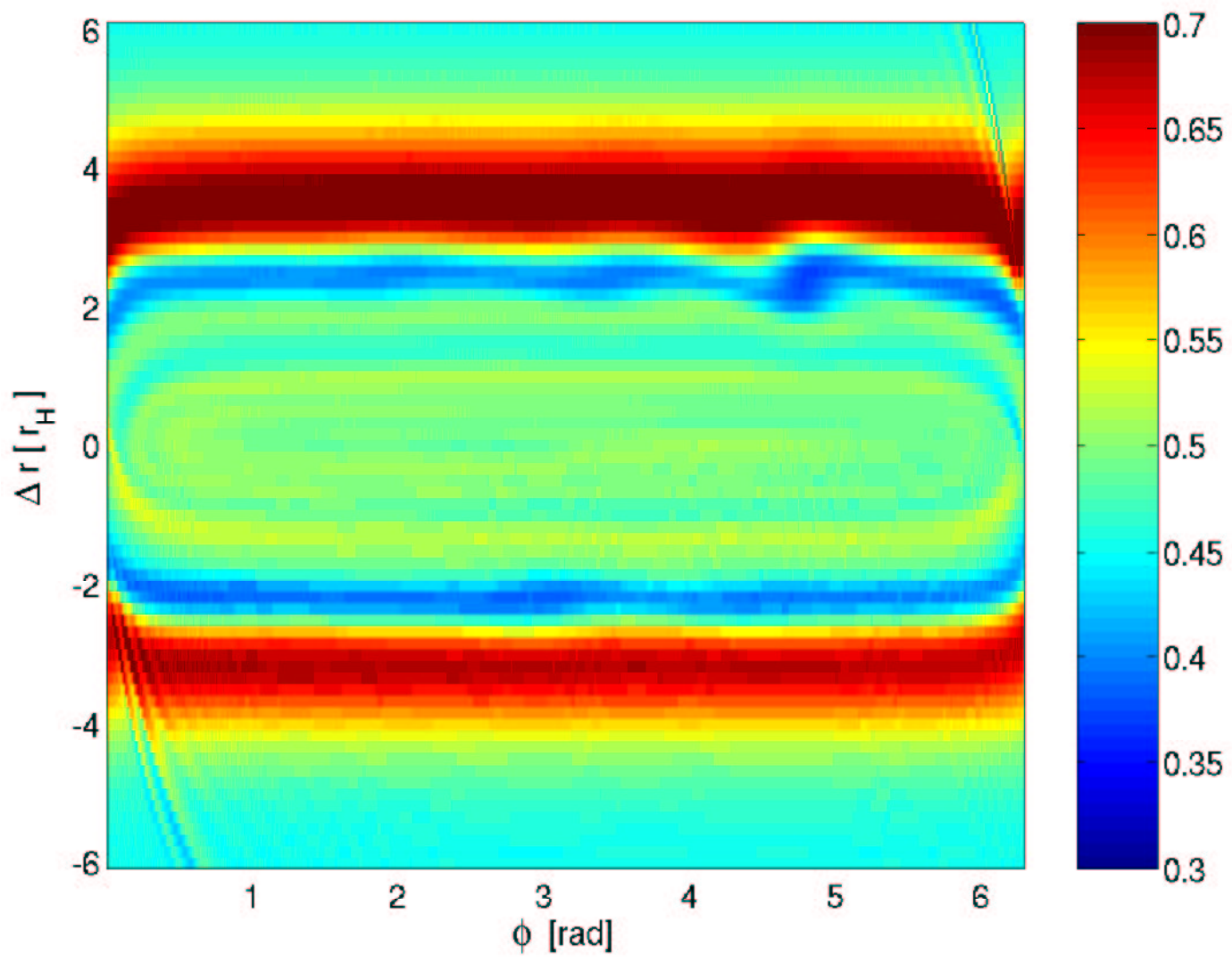

Figure 6.3: Potential vorticity $\zeta$ showing the production of vortices (same as Fig. 6.2). At $t=90 P$ the outer channel shows emerging vortices (anti-cyclones). 


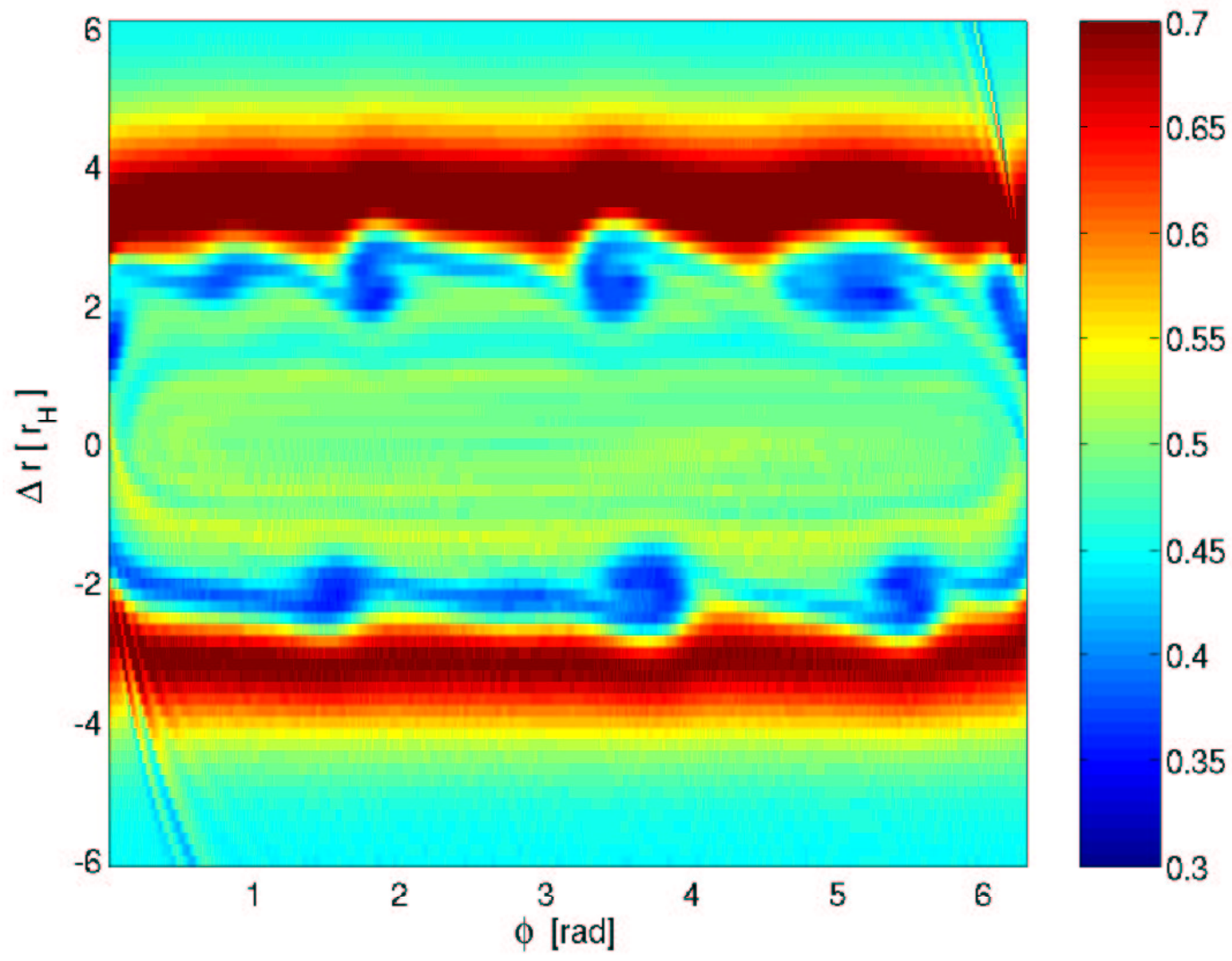

Figure 6.4: Potential vorticity $\zeta$ showing the production of vortices (see also Fig. 6.2 and 6.3). At $t=100 P$ vortices have grown to their full size in both potential vorticity channels. Note that vortices are moving in the separatrix region, so they will experience close-encounters with the planet. 


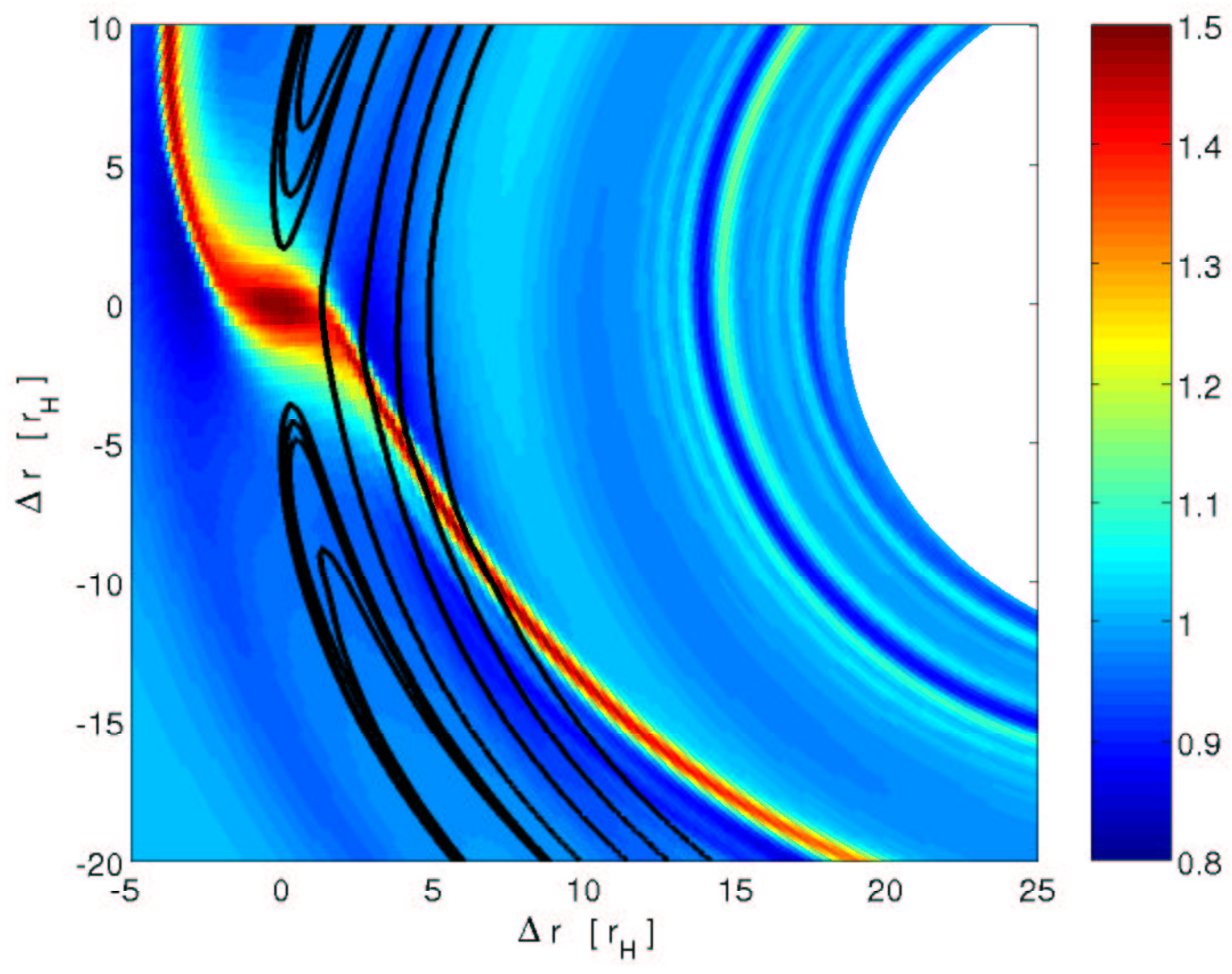

Figure 6.5: Density of a protoplanetary disk with an embedded plant. The axis are labeled in Roche lobe radii. The planet is located at $\left(r_{p}, \phi_{p}\right)=(0,0)$. The background density $\Sigma \propto r^{-3 / 2}$ has been divided out. Shock crossing and horseshoe flow lines (black) are shown as well. 


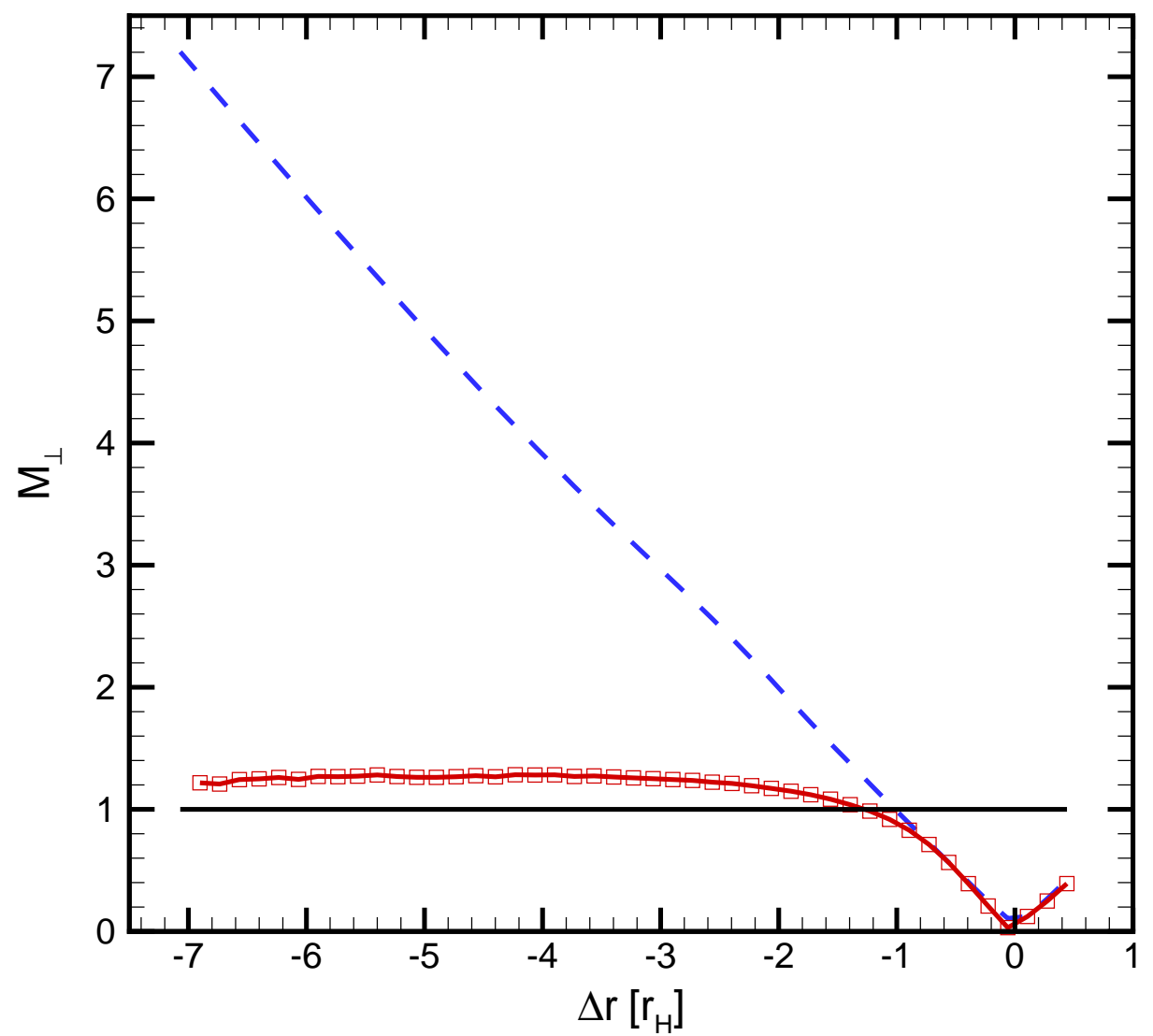

Figure 6.6: Shock structure in terms of Mach number. Taking into account the obliqueness of the shock and the increasing relative velocity (dashed line) to the shock front, the Mach number $M_{\perp}$ of this shock is very small. The supersonic region starts at around $-1.2 r_{H}$ and never exceed the $M_{\perp}=1.3$. The thick line denotes the supersonic regime where $M_{\perp}>1$. This figure is based on a simulation with $c_{s}=0.05$ and $\mu=10^{-4}$. 


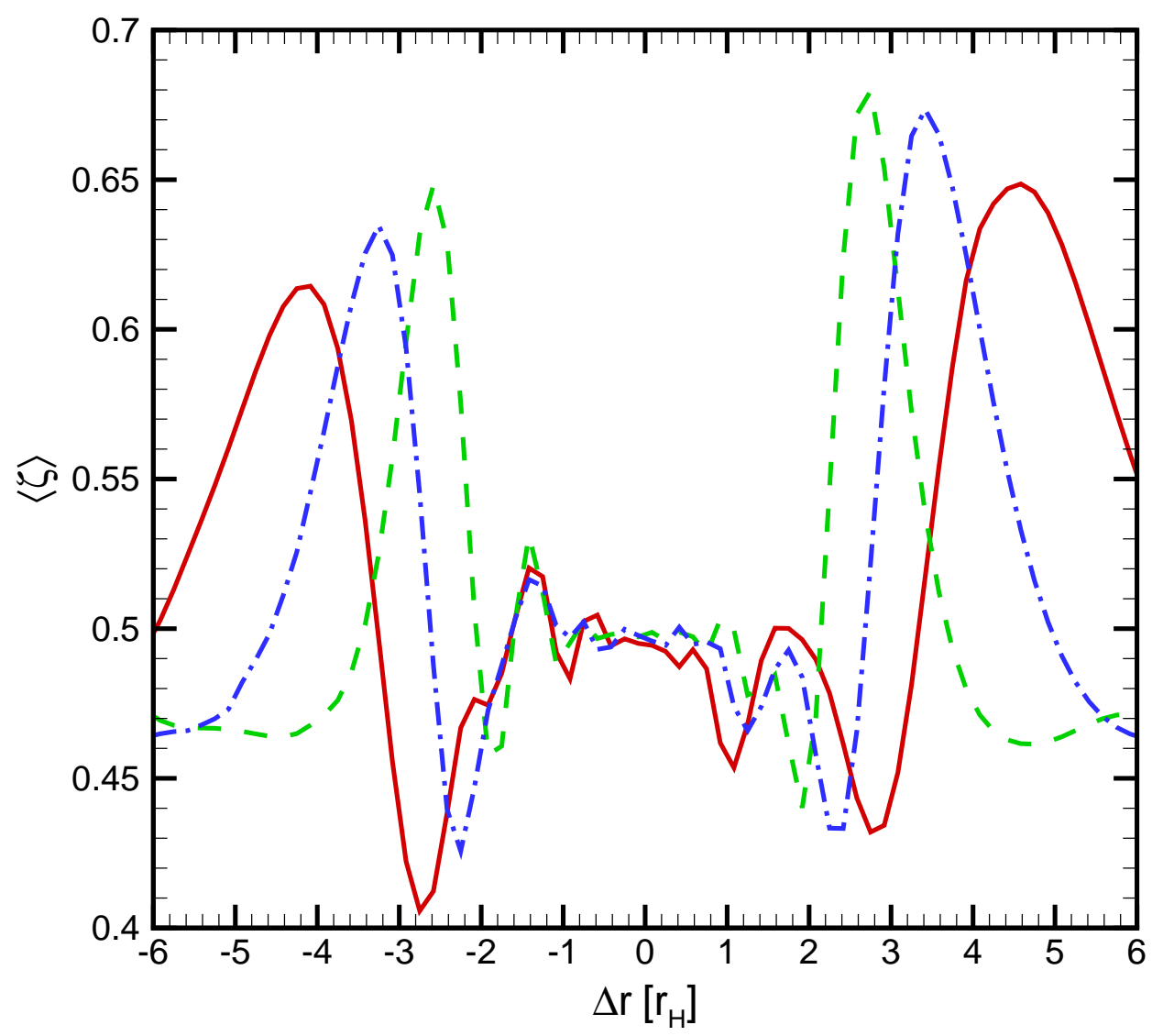

Figure 6.7: Azimuthally averaged potential vorticity $\langle\zeta\rangle$ shortly before vortices set in for different sound speeds $c_{s}=0.04$ (green solid), 0.05 (blue dash-dotted), 0.06 (red solid). The evolution for lower $c_{s}$ is much faster and therefore vortices develop early on. Vortices first appear at the minima of the profile. The locations of the minima move outwards proportionally as sound speeds increase, so do the starting locations of the spiral shocks. 


\section{Chapter 7}

\section{Parameter Studies}

\subsection{Key Parameter $K$}

A major finding of this research is that the ratio of sound speed $c_{s}$ over the planet mass $\mu$ is a significant key parameter to study the disk response. Let's define

$$
K=\frac{1.5 c_{s}}{\sqrt{12} r_{H}} .
$$

As discussed before, the planet produces spiral shocks, and the location or starting point can be changed with sound speed $c_{s}$ (Fig. 7.1). Also the planet mass $\mu$ can influence the starting point. Fig. 7.1 shows that for $\mu=10^{-4}$ and $c_{s}=0.05$ the shock starts roughly at $\Delta r= \pm 1.2 r_{H}$. That location changes with Eq. (7.1).

By studying several simulation, it can be found that $K$ determines where the shock is located and if it will be inside the horseshoe, in the separatrix, or in the 
streaming region (see $\S 12$ ). If $K>1$, the disk response is weak because the shock is located away from the planet in the streaming region. If $K<1$, the driving shock lies within the separatrix regions which is most receptive to instabilities.

To test the hypothesis that a constant $K$ will have the same disk response, simulations with $c_{s}=0.06$ and $\mu=1.728 \times 10^{-4}$ and another run with $c_{s}=0.05$ and $\mu=10^{-4}$ were made. Therefore, $K$ is the same for both runs. Fig. 7.2 depicts the torque

$$
T_{d}=\int\left[r_{p} \times F_{d}(r, \phi)\right]_{z} r d r d \phi
$$

from these runs, where $F_{d}(r, \phi)$ is the gravitational force of the disk at $(r, \phi)$. In both runs we see a smooth torque between $10 P<t<80 P$, which we call phase I, and a "noisy" torque for $t>80 P$, which we call phase II (see $§ 5.2$ for details). This figure also shows that the transition to phase II occurs at about the same time $t \approx 80 P$ for both runs.

Also the azimuthally averaged potential vorticity profiles $\langle\zeta\rangle$ (Fig. 7.3) are almost identical. This is expected since the secondary instabilities develop at the same time and at the same location. One can conclude that as long as $K$ is constant, the disk response, including growth rates for instabilities, is the same.

We also note in Fig. 7.3 that the oscillation period in phase I decreased from $P_{I}\left(\mu=10^{-4}\right) \approx 40 P$ to $P_{I}\left(\mu=1.7 \times 10^{-4}\right) \approx 30 P$. In general, the period in phase I is due to particles in horseshoe and tadpole orbits. Since their libration period 
$T_{L} \propto 1 / \sqrt{\mu}$ (see $\left.\S 12.1\right)$, we find that the period changes with

$$
\frac{P_{1}}{P_{2}}=\sqrt{\frac{\mu_{2}}{\mu_{1}}} .
$$

However, it should be noted here that not only the libration period is responsible for the oscillation period in phase I, but rather a combination of periods of particles in the horseshoe region. This will be discussed further in $\S 12$.

\subsection{Torque Evolution and Sound Speed Depen- dence}

In Fig. 7.4 we see the total torque $T_{d}(t)$ for all sound speed simulations between $c_{s}=0.02$ and up to $c_{s}=0.1$ with a constant $\mu=10^{-4}$. We can divide the sound speed simulations into three distinct groups, all with a different torque response. They are grouped into $c_{s}<0.04$ (low), $0.04 \leq c_{s} \leq 0.06$ (middle), and $c_{s}>0.06$ (high). This result can be understood with the proposed key parameter $K$ and the location of shocks. Fig. 7.1 describes where the shock starts depending on the sound speed. For lower sound speed the shock is not only stronger but also starts closer to the planet. High sound speeds weaken the shock with a starting point much further away from the planet.

For the low sound speed runs $\left(c_{s}<0.04\right)$ the starting point of the spiral shocks 


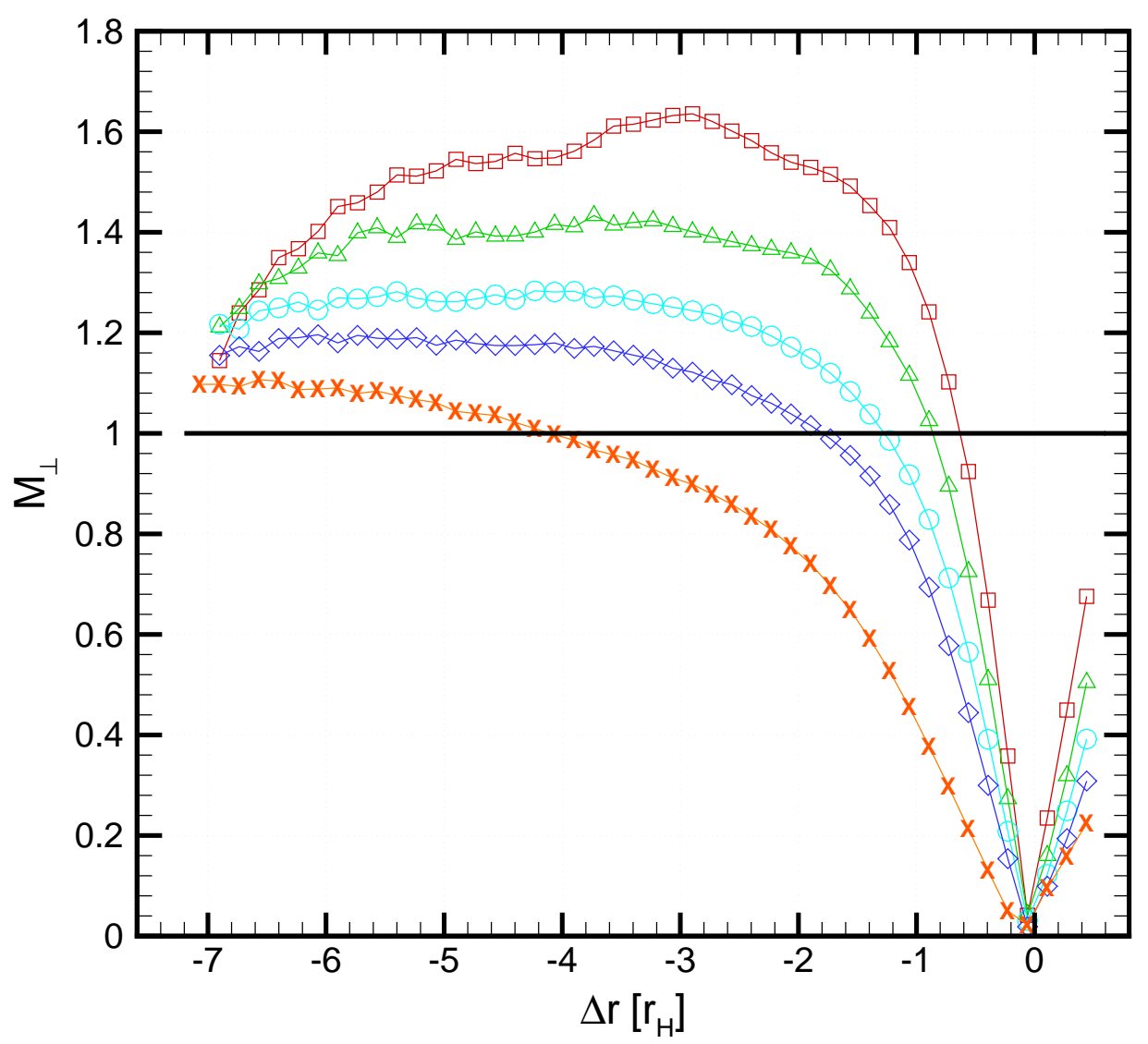

Figure 7.1: Shock structure in terms of Mach number for different sound speeds. The symbols denote measurements from runs with $c_{s}=0.032$ (squares), $c_{s}=0.04$ (triangles), $c_{s}=0.05$ (circles), $c_{s}=0.06$ (diamonds), $c_{s}=0.1$ (crosses). The thick line shows where the supersonic regions lies. The shock starts at a larger distance from the planet for a higher sound speed. 


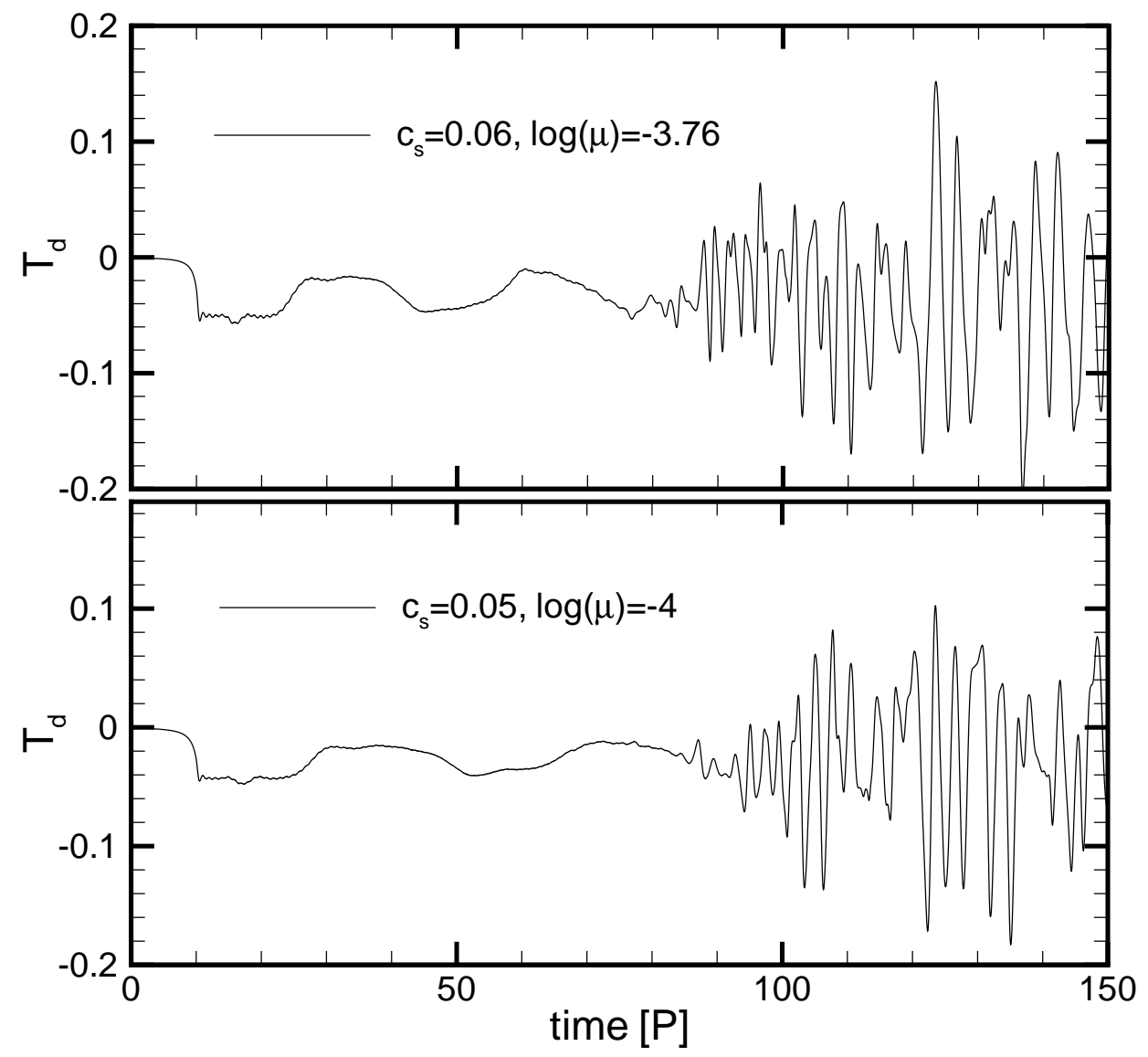

Figure 7.2: Torque evolution of two simulations with constant key parameter $K$ (Eq. 7.1). The upper plot shows the torque $T_{d}(t)$ from a simulation with $c_{s}=0.06$ and $\mu=1.728 \times 10^{-4}$. The lower plot depicts a simulation with $c_{s}=0.05$ and $\mu=10^{-4}$. Therefore, both runs have the same $K$ value. A turn-on time for the planet mass of 10 orbits was used. In phase I the torque is negative and smooth and the transition into phase II occurs at roughly the same time $t=80 P$ for both runs with the same $K$. 


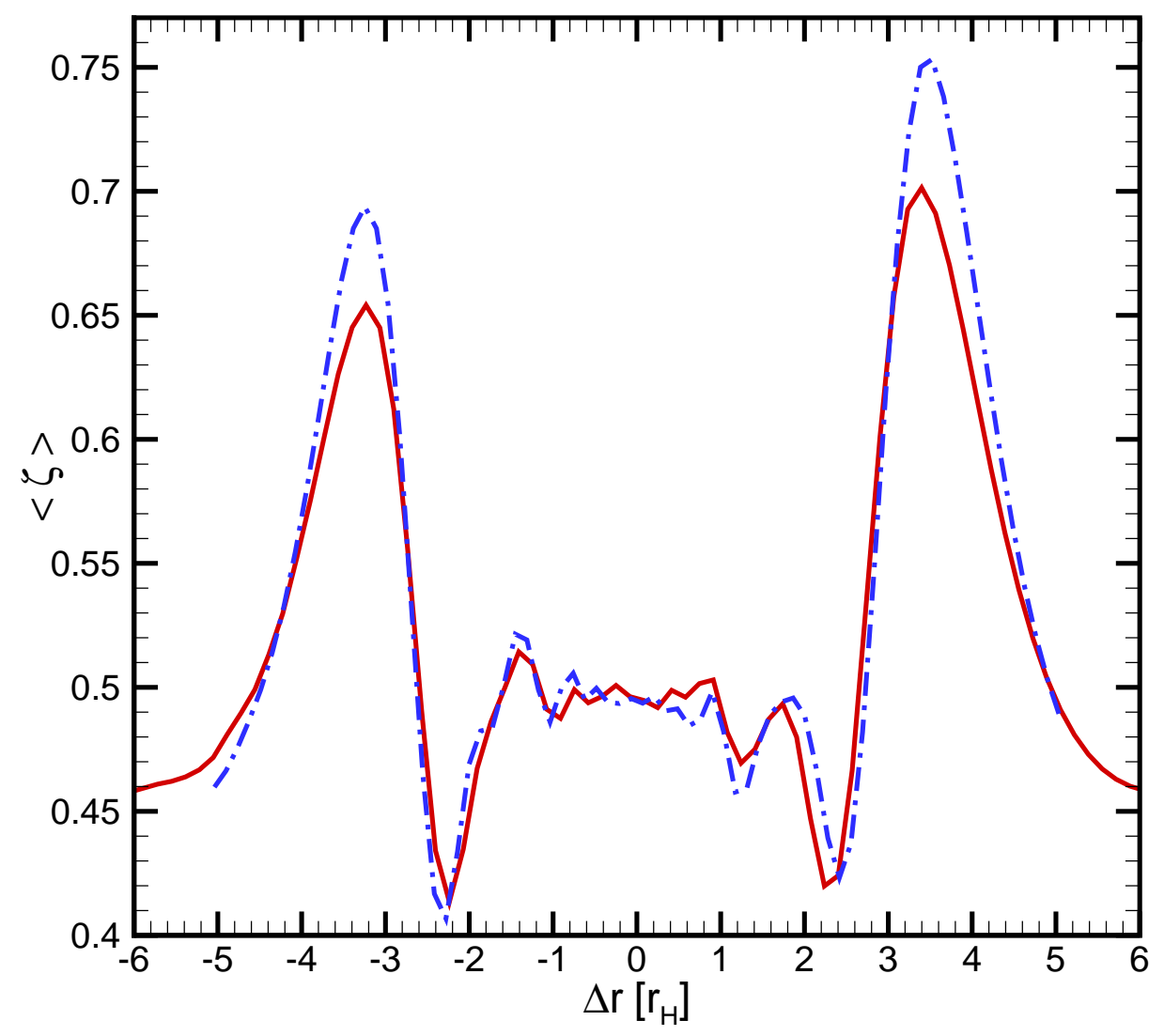

Figure 7.3: Azimuthally averaged PV profile at $t=80 P$ shortly before vortices emerge. This is a comparison of two simulations with a constant key parameter $K$. The minima in the PV profile match in location and depth. As a result the secondary instabilities develop at the same time. The blue dash-dotted line is for the simulation with $c_{s}=0.06$ and $\log (\mu)=-3.76$. The red solid line has the parameters $c_{s}=0.05$ and $\log (\mu)=-4$. 
is at $\left|\Delta r_{s}\right|<r_{H}$ and lies therefore within the horseshoe region. The parameter $K<1$ for this range. These runs do not show a distinct phase I but a strong phase II which is developing early on. Looking at $c_{s}=0.02$ and 0.032 in Fig. 7.4, the transition to phase II occurs so early in the simulation that no libration period can be observed. The disk shows vortices of smaller size at $|\Delta r| \approx 1.5 r_{H}$. Again, the $\langle\zeta\rangle$ profile develops inflection points with minima which match the $\Delta r$ location for vortices. Fig. 7.5 describes how the density for a $c_{s}<0.04$ decreases down to $60 \%$ of the initial density and creates a sharp peak in the potential vorticity profile. The PV profile depicts almost no distinct minima. Still some vortices are created but the overall torque evolution is not as strong and not as distinctly divided into phase I and II as for the group of medium sound speeds.

The middle sound speed group $\left(0.04 \leq c_{s} \leq 0.06\right)$ has a stronger behavior in term of torque evolution, vortices are bigger, and phase I and phase II are clearly separated. Also the shocks are located in the separatrix region. These runs have a phase I with an oscillation period similar to the libration period of particles in the horseshoe area.

Fig. 7.6 illustrates how the location of the minima moves to a large $|\Delta r|$ with increasing sound speed. All three runs show that strong vortices emerge at $|\Delta r| \approx 1.8 r_{H}, 2.2 r_{H}, 3 r_{H}$ for $c_{s}=0.04,0.05,0.06$. This behavior agrees with our earlier statement that shocks are causing the development of inflection points in the potential vorticity profile and their location determines where secondary 
instabilities emerge.

For the high sound speed runs $\left(c_{s}>0.06\right)$ with $K>1$ the disk shows only a weak response to the planet (Fig. 7.7). The torque $T_{d}(t)$ develops an almost constant level (see Fig. 7.4). Note the ordinate is the same for all torque figures. These runs also have a phase transition but the area is constrained to a much smaller band at $|\Delta r| \approx r_{H}$. Zooming closer in, we still see a phase transition and some small scale vortices in the horseshoe region. Due to their specific orbital motion, they don't come as close to the planet and therefore the torque amplitudes are much smaller.

A closer inspection of $\mathrm{PV}$ images of runs with $K \approx 1$, show that a smooth transition takes place between the middle and high sound speed group. Here, some small scale vortices emerge in the horseshoe region similar to the vortices in Balmforth \& Korycansky (2001) probably due to PV mixing in this region. The dip in the $\langle\zeta\rangle$ profile moves further away from the planetary orbit to a larger $|\Delta r|$ with an increased width and a lower depth, which makes the dip less receptive to secondary instabilities.

The transition time to phase II depends on the sound speed and is plotted in Fig. 7.8 where high $c_{s}$ runs have a much slower growth rate and therefore secondary instabilities develop at a much later time. 


\subsection{Growth Rate Parameter}

Based on Fig. 7.6, a working hypothesis is suggested that vortices are the nonlinear outcome of an instability which is excited at the extrema in the $\langle\zeta\rangle$ profile when such extrema exceed a certain threshold. There are two ways to quantify the growth of the potential vorticity profile and the threshold conditions: (1) Let's pick the minimum at $\Delta r \sim 2 r_{H}$ (for $c_{s}=0.05$ ) and define the change of $\langle\zeta\rangle$ as $\Delta C=\zeta_{0}-\zeta_{\min }(t)$, where $\zeta_{0}=0.497$ for this $\Delta r$ and $c_{s}$.

A different way (2) for looking at the $\langle\zeta\rangle$ evolution is to take the double derivative of the $\langle\zeta\rangle$ profile and search for the maximum $\eta$ which is located at a $\Delta r$ where the vortices emerge (Fig. 7.9). Both methods show a linear growth until they reach a threshold when the secondary instabilities develop. The advantage of the second method is that it contains also information about the width and the depth of the $\langle\zeta\rangle$ minimum.

Both parameters $\eta\left(\mu, c_{s}\right)$ and $\Delta C\left(\mu, c_{s}\right)$ depend on sound speed and planet mass. The functional dependence on $\mu$ and $c_{s}$ is investigated in the following section.

\subsection{Functional Dependence on Planet Mass}

To determine the functional dependence for $\eta\left(\mu, c_{s}\right)$ on sound speed and planet mass, we can start with keeping the sound speed constant. Fig. 7.11 shows the 
growth rate for $\Delta C(\mu)$ with constant sound speed $c_{s}=0.05$ for several planet masses between $9 \times 10^{-6}<\mu<10^{-4}$. We can find an empirical relation

$$
\Delta C=\int d t\langle\dot{\zeta}\rangle=\int d t A \mu(t)^{2}
$$

i.e. the growth rate is a function of $\mu^{2}$. This is true for both a $\mu$ and $\mu(t)$.

From the growth rate $\langle\dot{\zeta}\rangle$ and together with the observed threshold $\Delta C_{t r} \approx 0.1$, one can derive a time-scale for emerging vortices, $\tau_{v o r t} \approx \Delta C_{t r} / \dot{C}$ (orbits), where $A \approx 10^{5}$. In other words, for a planet with $\mu=10^{-4}, \tau_{v o r t} \approx 100$ orbits.

To study effects of a growing planet mass, we can create a turn-on expression depending on the libration period of a particle in the horseshoe region $\tau_{l}=2 \pi / \lambda$ where $\lambda^{-1}$ is in units of orbital periods at $r=r_{p}$. The orbital period of such a particle, closely orbiting one of the libration points, is $\lambda=(27 \mu / 4)^{1 / 2}$ (see §12.1). A procedure was developed to turn-on the planet slowly enough so the disk would eventually evolve at an adiabatic limit.

The question here is what is the effect of the time scale for the planet mass increase. In case the planet is turned on very slowly, will the disk evolution become adiabatic? Will the potential vorticity profile still develop inflection points which eventually evolve into secondary instabilities? Or is the time-scale a minor factor for creating the observed potential vorticity profile but rather an intrinsic necessity for a disk with a planet. 
To find a turn-on time which is slower than the libration period, we can write

$$
\tau_{o n}=\frac{\mu}{\dot{\mu}}=n \tau_{l}=\frac{2 \pi n}{\lambda}
$$

where $n>1$ to satisfy the turn-on condition for being slower than the libration period. Solving this equation and integrating over time gives

$$
\mu(t)=\frac{\mu_{i}}{\left(1-t \sqrt{27 \mu_{i}} / 8 n \pi\right)^{2}}
$$

with $\mu_{i}=\mu(0)$ as the initial planet mass. Solving (7.6) for $t$ to reach the final mass $\mu_{f}$ we obtain

$$
t_{f}=\frac{8 n \pi}{\sqrt{27 \mu_{i}}}\left[1-\left(\sqrt{\frac{\mu_{i}}{\mu_{f}}}\right)\right] .
$$

We can also write the mass increase of the planet as

$$
\mu(t)=\frac{\mu_{i}}{\left[1-\left(1-\sqrt{\mu_{i} / \mu_{f}}\right) t / t_{f}\right]^{2}}
$$

For the initial mass $\mu_{i}=10^{-6}$ and the final mass $\mu_{f}=10^{-4}$, we can get the turn-on time $t_{f} \approx 2 \times 700 n \pi$. For $n=1$ and a final mass of $\mu_{f}=10^{-4}$, the turn-on of the planet has to occur over 700 orbits. Since this is quite a long time for simulations, we can also somewhat increase the initial planet mass to reduce the turn-on time but still be slower than the libration period. A larger $\mu_{i}$ will 


\begin{tabular}{cc}
\hline \hline$\mu_{i}$ & $t_{f} /(2 n \pi)$ \\
\hline $1 \times 10^{-6}$ & 697 \\
$3 \times 10^{-6}$ & 367 \\
$1 \times 10^{-5}$ & 167 \\
$1.6 \times 10^{-5}$ & 116 \\
$2.5 \times 10^{-5}$ & 77 \\
$3.6 \times 10^{-5}$ & 52 \\
\hline
\end{tabular}

Table 7.1: Turn-on times for different initial mass ratios

introduce a larger impulse to the disk in the beginning but it has an important advantage: It will change $t_{f}$ to a practical time for simulations (see also Table 7.1).

The simulations clearly demonstrate that structures in the radial PV profile are always developed in the area between the streaming region and the horseshoe region $r_{H}<|\Delta r|<\sqrt{12} r_{H}$. The transition to secondary instabilities occurs when the radial potential vorticity profile develops extrema (i.e., inflection points) and such extrema exceed a certain threshold.

We can apply the mass turn-on procedure, which has been derived above, adopting $\mu_{i}=10^{-6}, \mu_{f}=10^{-4}$, and using Eq. (7.8). Choosing several turn-on times with $t_{f}=100,180,300,400,500,700$ orbits, we find the result shown in Fig. 7.10 for $c_{s}=0.05$. After the turn-on time is over and the planet mass is fixed, the parameter $\Delta C$ growths linearly with time until it reaches a value $\Delta C_{t r}<0.1$. At this point potential vorticity images show emerging vortices which fill up the minimum in $\langle\zeta\rangle$ and therefore $\Delta C$ turns over. Therefore, independent on how fast the planet reaches its final mass, the potential vorticity profile develops an inflection point until $\Delta C \approx 0.1$ is reached and secondary instabilities form. 
Fig. 7.10 shows that choosing a different turn-on time is not significant for developing secondary instabilities, it will just defer it to a later time when $\langle\zeta\rangle$ reaches the proper threshold. The growth rate and the threshold is the same for all turn-on times after the planet reaches its final mass.

\subsection{Functional Dependence on Sound Speed}

With different sound speed simulations, we can find a more complicated functional dependence of $\eta\left(c_{s}\right)$ or $\Delta C\left(c_{s}\right)$. As it was mentioned earlier, the results from the simulations with changing sound speed can be divided into three different groups which can be understood with the parameter $K$.

Fig. 7.12 illustrates the slope of $\Delta C\left(c_{s}\right)$. After the planet reaches the final mass and the disk had an appropriate amount of time to respond to the perturber, the functional dependence is linear with time and can therefore be written as

$$
\Delta C=B\left(c_{s}, \mu\right) t
$$

where $B\left(c_{s}, \mu\right)=d(\Delta C) / d t$. We see a distinct knee in Fig. 7.12 when the sound speed is approximately the disk scale height $c_{s} \approx H$. Then the shock location moves into the horseshoe region $\left|\Delta r_{s}\right|<r_{H}$ which has a slower growth rate and also produces smaller vortices. Furthermore, these vortices move in horseshoe orbits with more distant planet encounters (see $\S 12.1$ ). A constant $\mu=10^{-4}$ was 
chosen for all runs in Fig. 7.12.

Fig. 7.13 depicts $\eta(t)$ for the middle sound speed range. The turn-on time for these runs is $t_{f}=10 P$. We find it takes another ten orbits for the disk to respond to the perturber and after that we have again a linear growth. Interesting to note, the threshold for all runs can be described with a power law (dashed line in Fig. 7.13) $\tau_{\text {vort }} \approx 5.3 \times 10^{4} c_{s}^{-0.8}$. This relationship represents the threshold only for the intermediate sound speed runs. The lower sound speed runs develop the instabilities so early on that the a growth-rate cannot be extracted with much confidence. And the high sound speed simulations don't develop major vortices. However, they do show some small scale vortices at $|\Delta r| \approx r_{H}$ but their threshold does not follow this functional dependence. 

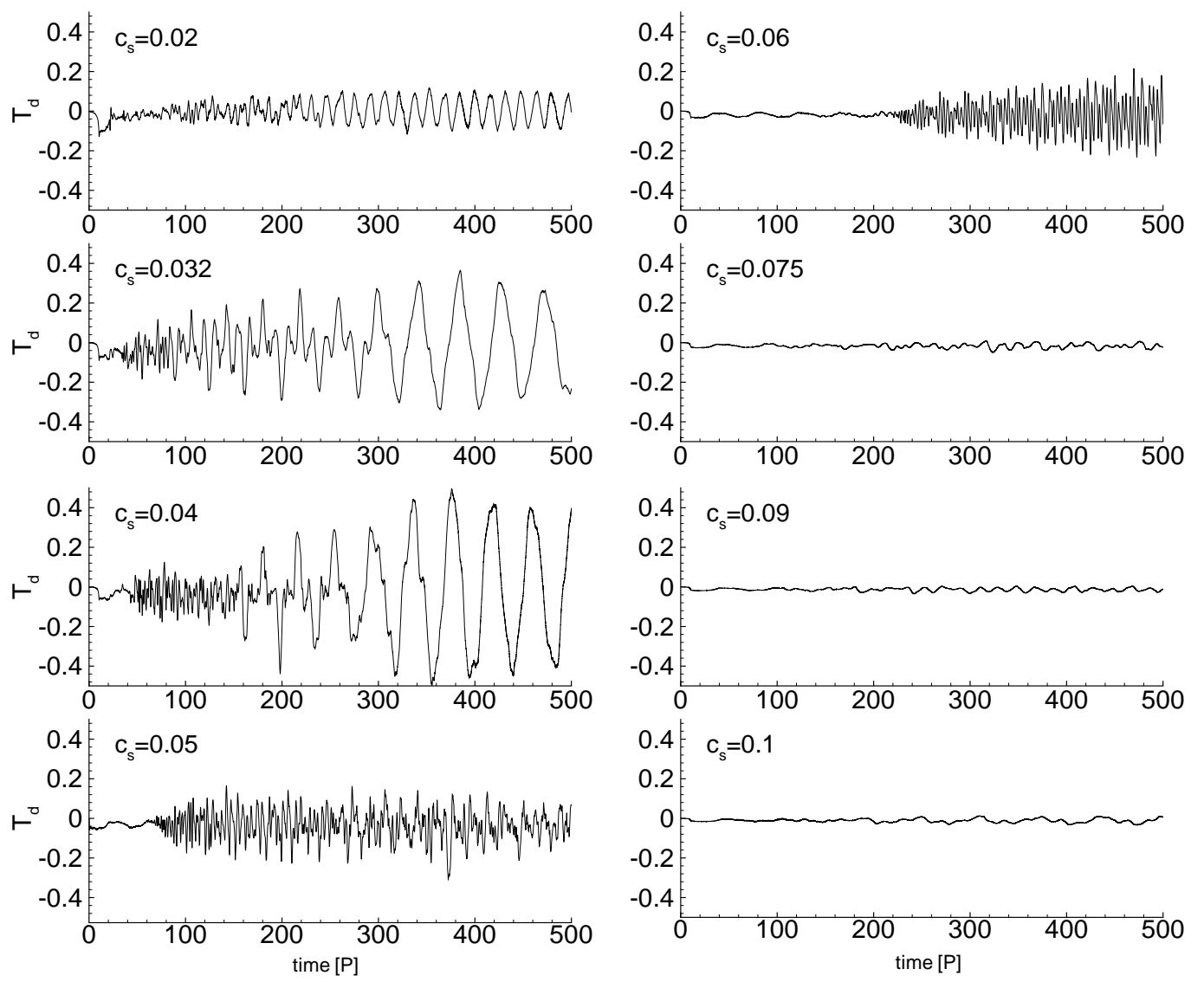

Figure 7.4: Torque evolution of several sound speed simulations from $c_{s}=0.02$ to 0.1 for the planet mass $\log (\mu)=-4$. The scaling is the same for all diagrams. The lowest sound speed (upper left) shows a phase I transition very early on and then later the merging of vortices (phase III). The intermediate sound speed runs from $c_{s}=0.04$ to 0.06 show a clear phase I and phase II in the torque evolution. The high sound speed simulation (lower right) also have a phase transition but the unstable area is constrained to a small band at $|\Delta r| \approx r_{H}$. The torques are small and have smaller amplitudes than in the lower sound speed simulations. 

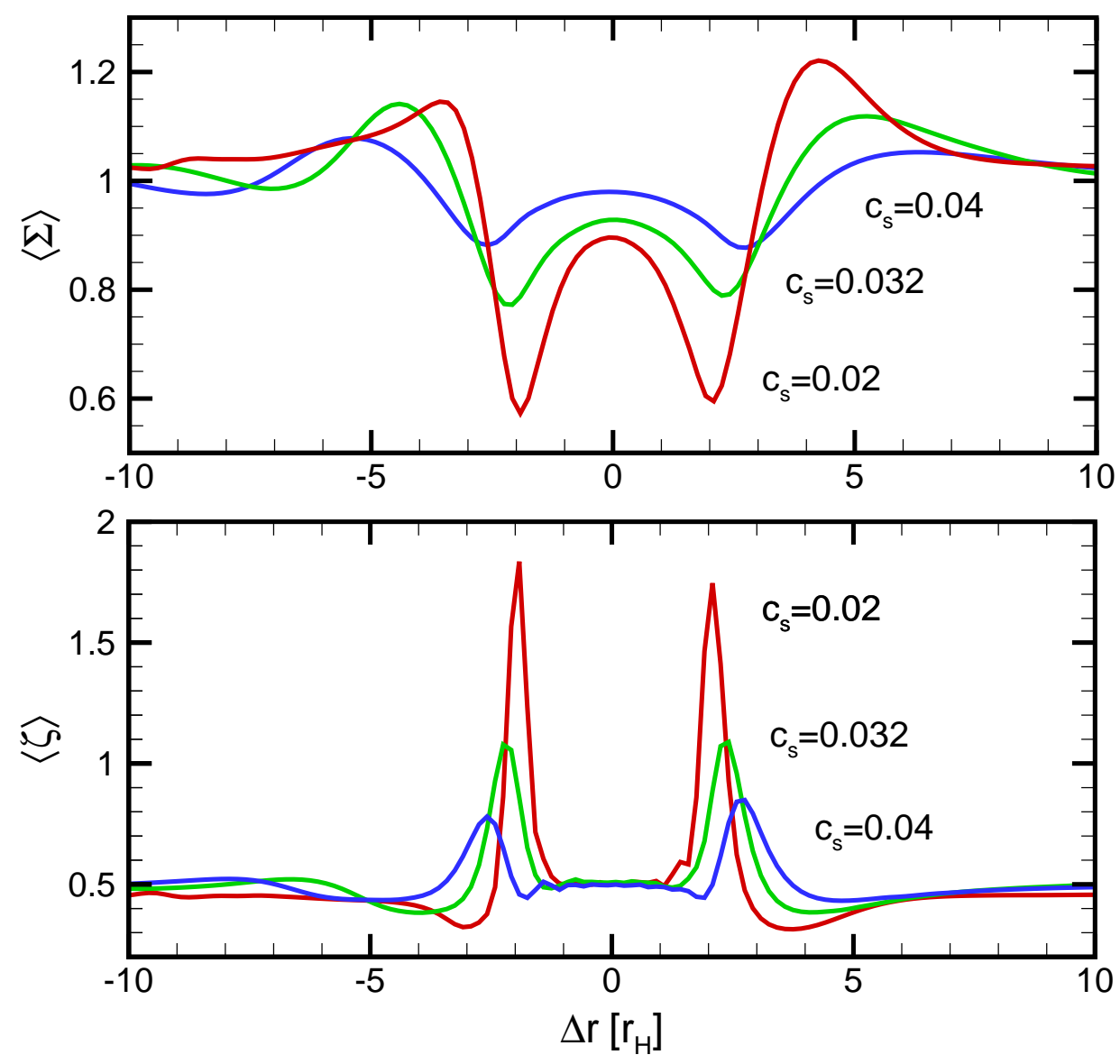

Figure 7.5: Azimuthally averaged surface density $\langle\Sigma\rangle$ (top) and potential vorticity $\langle\zeta\rangle$ (bottom) for three different sound speeds $c_{s}=0.02$ (red), $c_{s}=0.032$ (green), and $c_{s}=0.04$ (blue) at $t=50 P$. The density dips are responsible for the peaks in the potential vorticity profile since $\zeta \propto 1 / \Sigma$. 

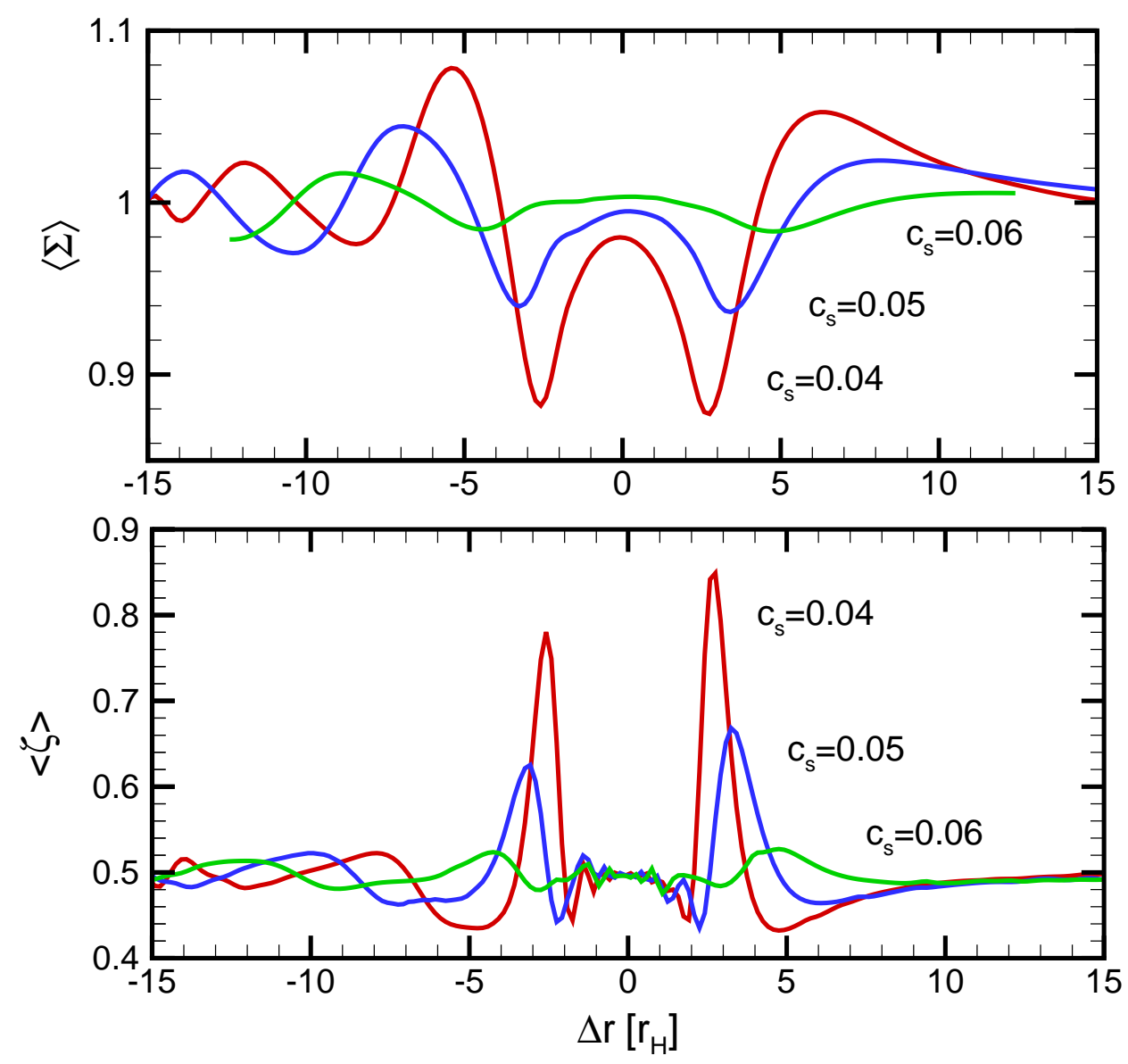

Figure 7.6: Azimuthally averaged surface density $\langle\Sigma\rangle$ and potential vorticity $\langle\zeta\rangle$ at $t=50 P$ in the mid sound speed regime at $c_{s}=0.04$ (red), $c_{s}=0.05$ (blue), and $c_{s}=0.06$ (green). The density dips are not as strong as for the lower sound speed runs but the potential vorticity shows clear dips where vortices can form. 

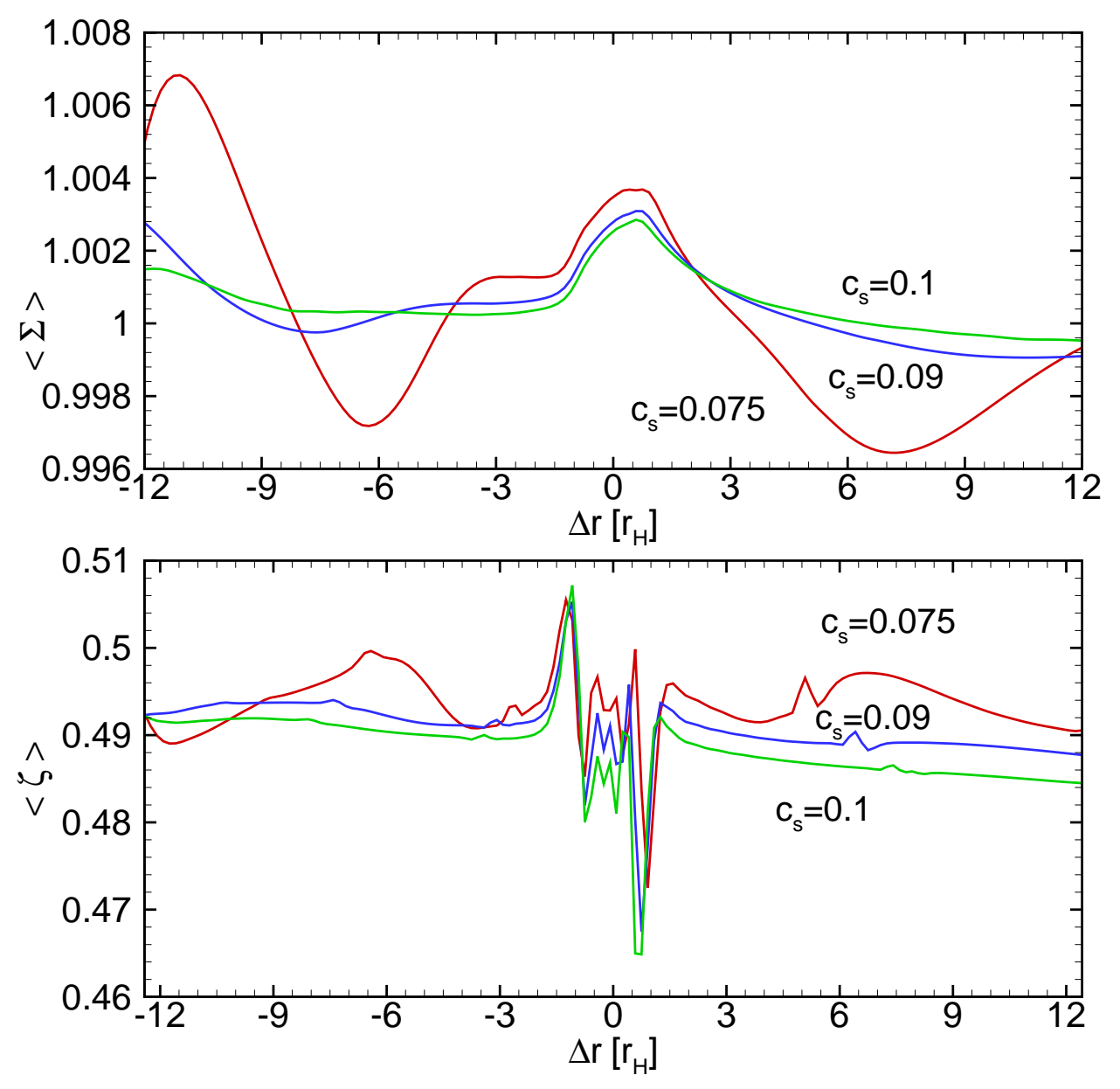

Figure 7.7: Azimuthally averaged surface density $\langle\Sigma\rangle$ and potential vorticity $\langle\zeta\rangle$ at $t=50 P$ for high sound speeds $c_{s}=0.075$ (red), $c_{s}=0.09$ (blue), and $c_{s}=0.1$ (green). This figure is the same as Fig. 7.5 and Fig. 7.6 but with different scaling. 


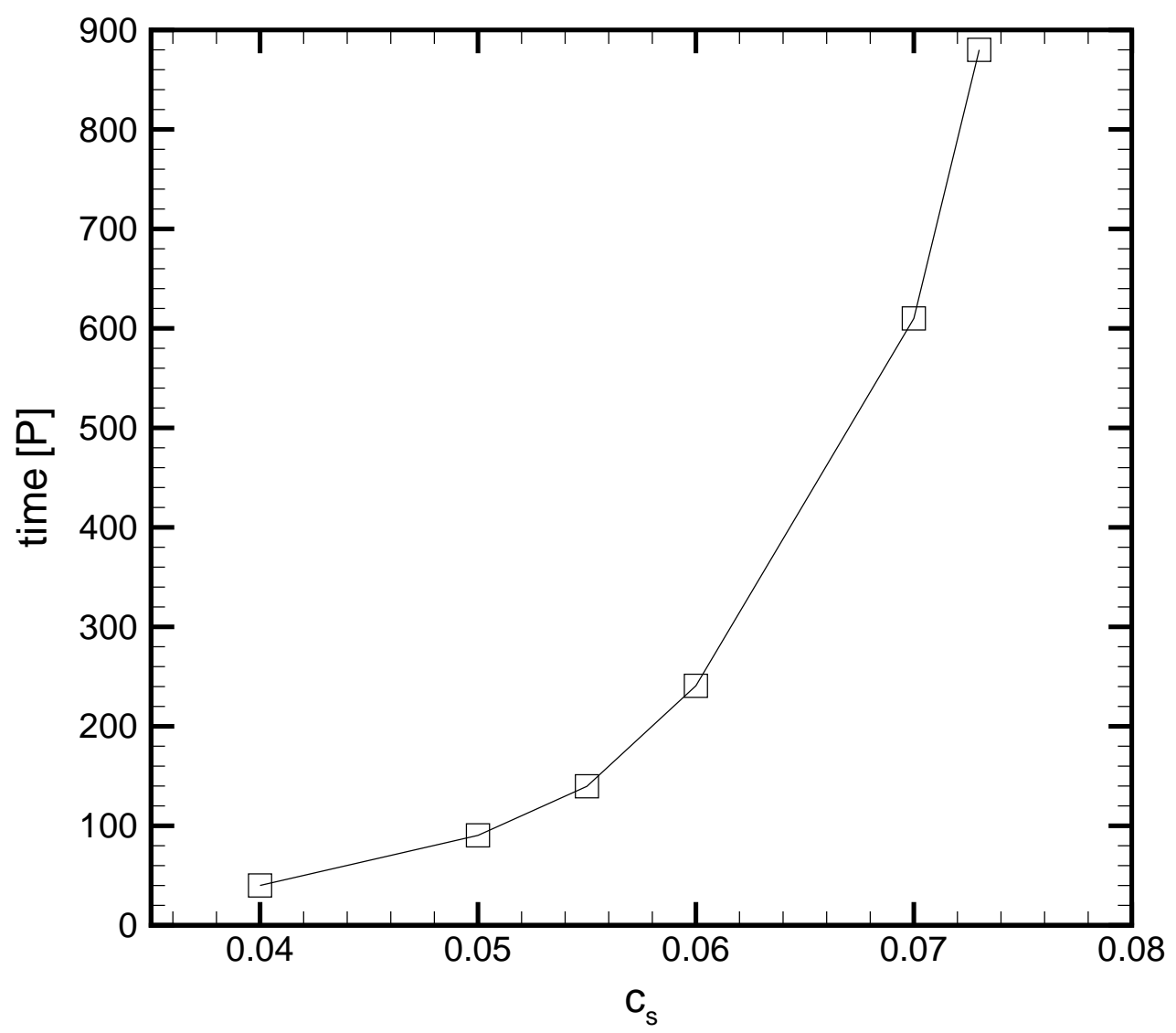

Figure 7.8: Time for the onset of secondary instabilities as a function of sound speed. When the minima in the potential vorticity profile become unstable, vortices form i.e. secondary instabilities develop. The time it takes from an initially smooth disk to develop unstable inflection points is a function of sound speed and planet mass. Here the planet mass is held constant at $\mu=10^{-4}$. Since the growth rate is smaller for higher sound speeds, the instabilities occur later on. 

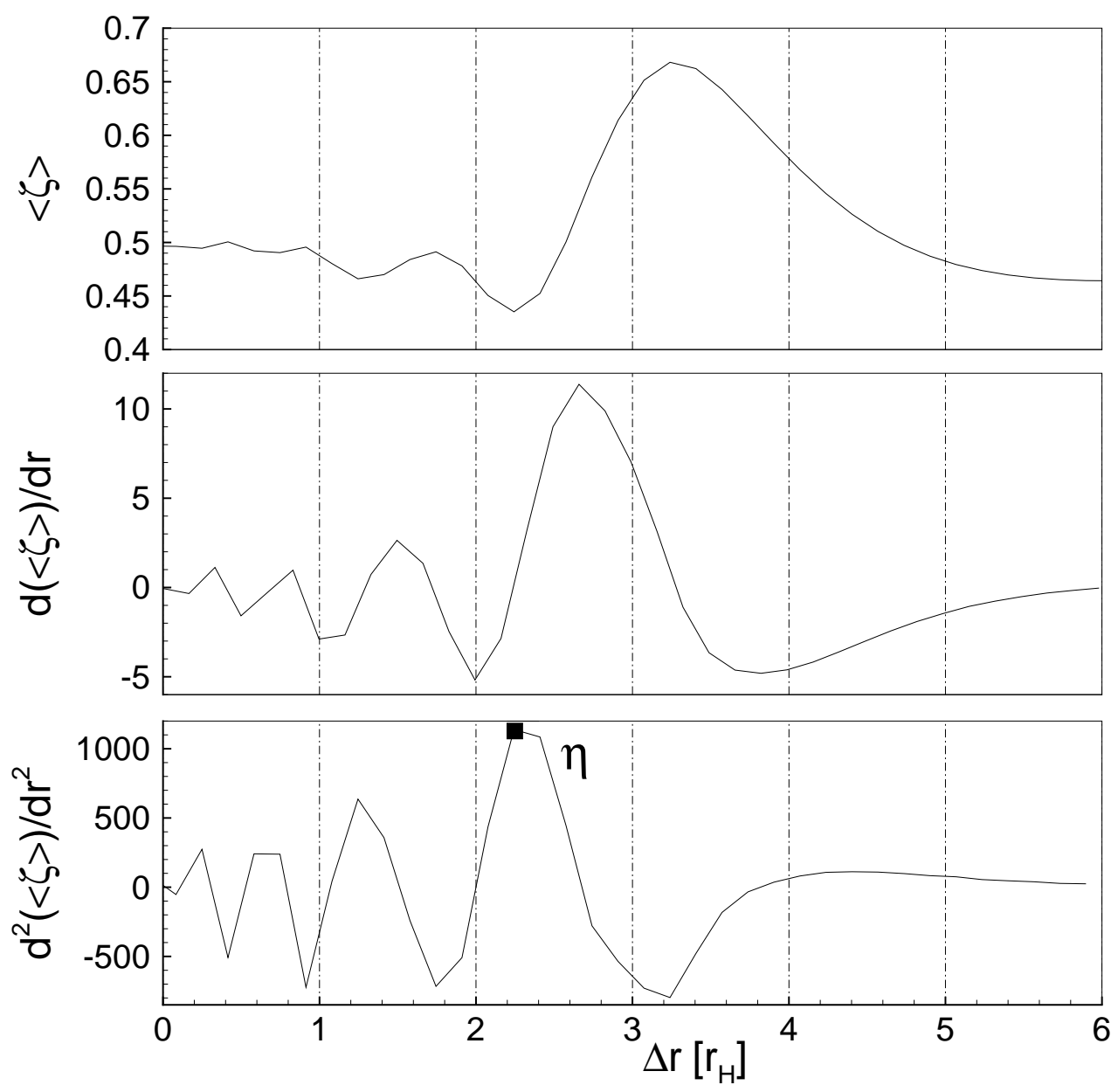

Figure 7.9: Azimuthally averaged PV profile $\langle\zeta\rangle$, its derivative and double derivative $d^{2}\langle\zeta\rangle / d r^{2}$ (here for $c_{s}=0.05$ at $t=50 P$ ). For method (2) we seek the maximum $\eta$ in $d^{2}\langle\zeta\rangle / d r^{2}$. This figure depicts only the outer half of the potential vorticity profile from $0<\Delta r<6 r_{H}$. 


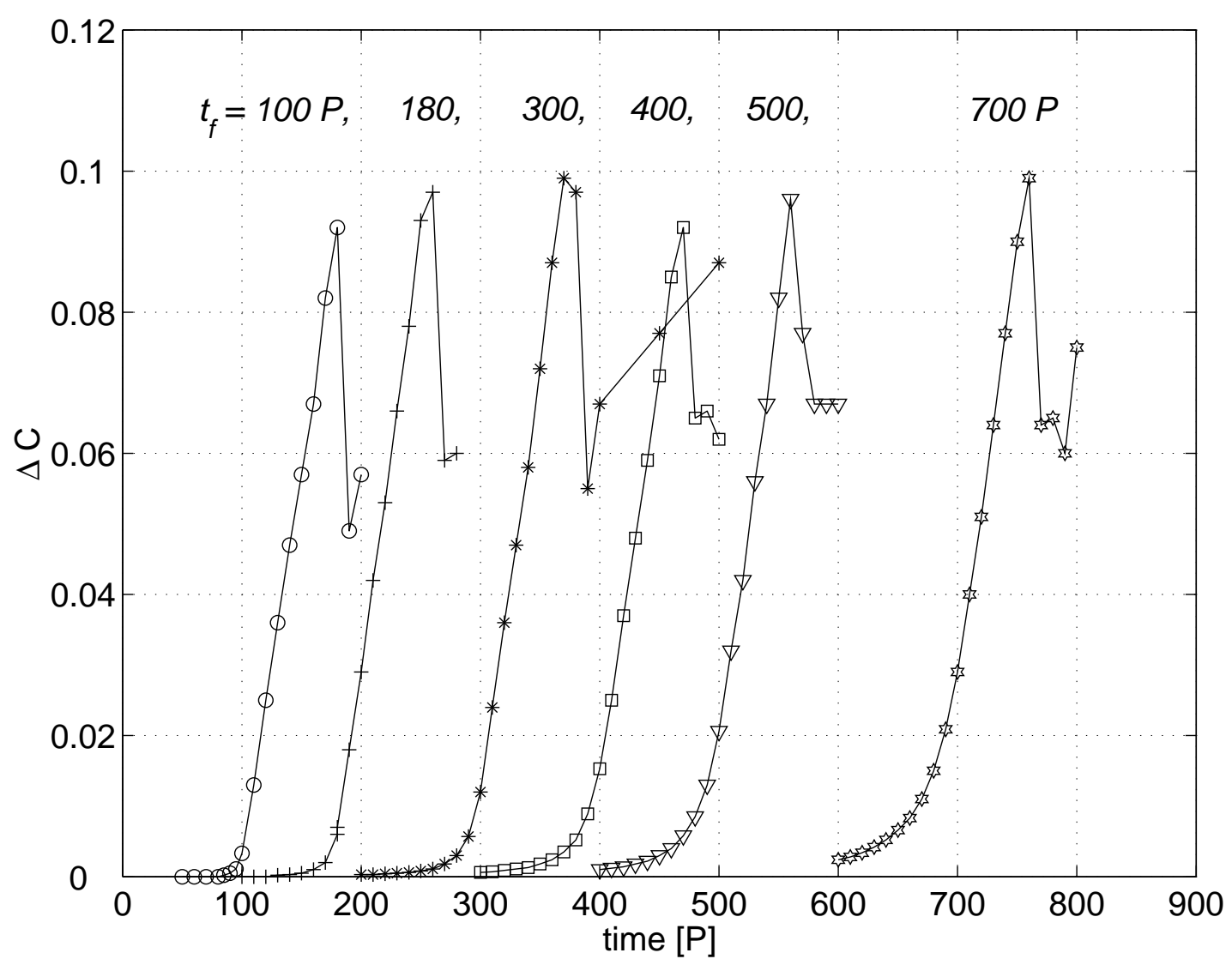

Figure 7.10: The growth of $\Delta C=\zeta_{0}-\zeta_{\min }$, where $\langle\zeta\rangle$ is the azimuthally averaged PV profile, as a function of time for different turn-on profiles (after Eq. 7.8). The initial mass is $\mu_{i}=10^{-6}$ and the final mass is $\mu_{f}=10^{-4}$. The threshold $\Delta C_{t r}<0.1$ holds for all turn-on profiles $t_{f}$, i.e. no matter how fast the planet reaches it's final mass, the PV profiles develop an inflection point until $\Delta C \approx 0.1$ is reached and secondary instabilities set in. We also note that the slope of $\Delta C$ is the same after the planet fully reached its mass, i.e., the growth rate for $\Delta C$ is independent of the turn-on profile. 


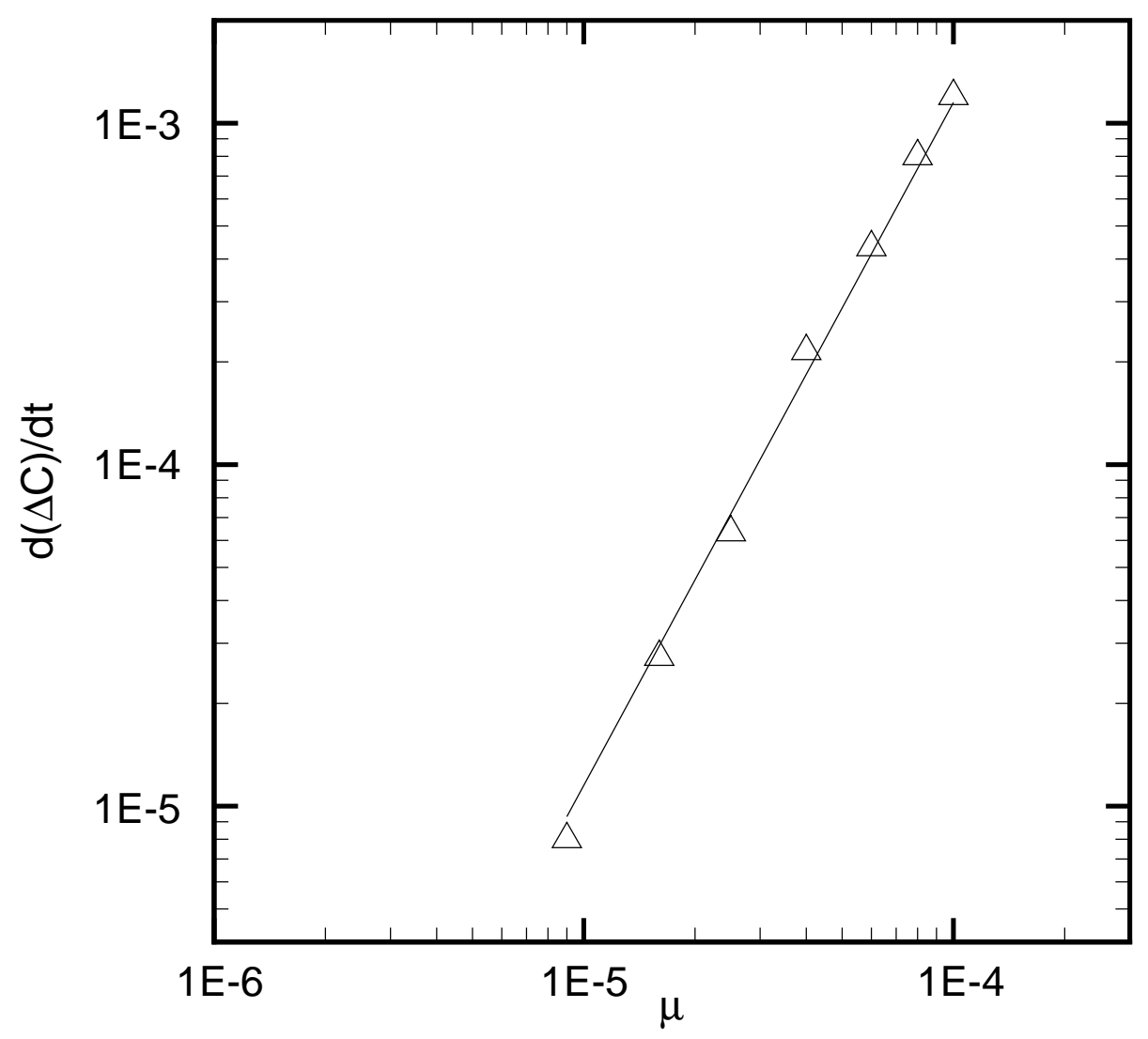

Figure 7.11: Growth rate for $\Delta C(\mu)$ with different constant planet masses. All simulations have the same sound speed $c_{s}=0.05$. The range of planet masses goes from $\mu=9 \times 10^{-6}$ to $\mu=10^{-4}$. Filled triangles are results from the simulations. The line represents a fit with $d(\Delta C) / d t=1.15 \times 10^{5} \mu^{2}$. 


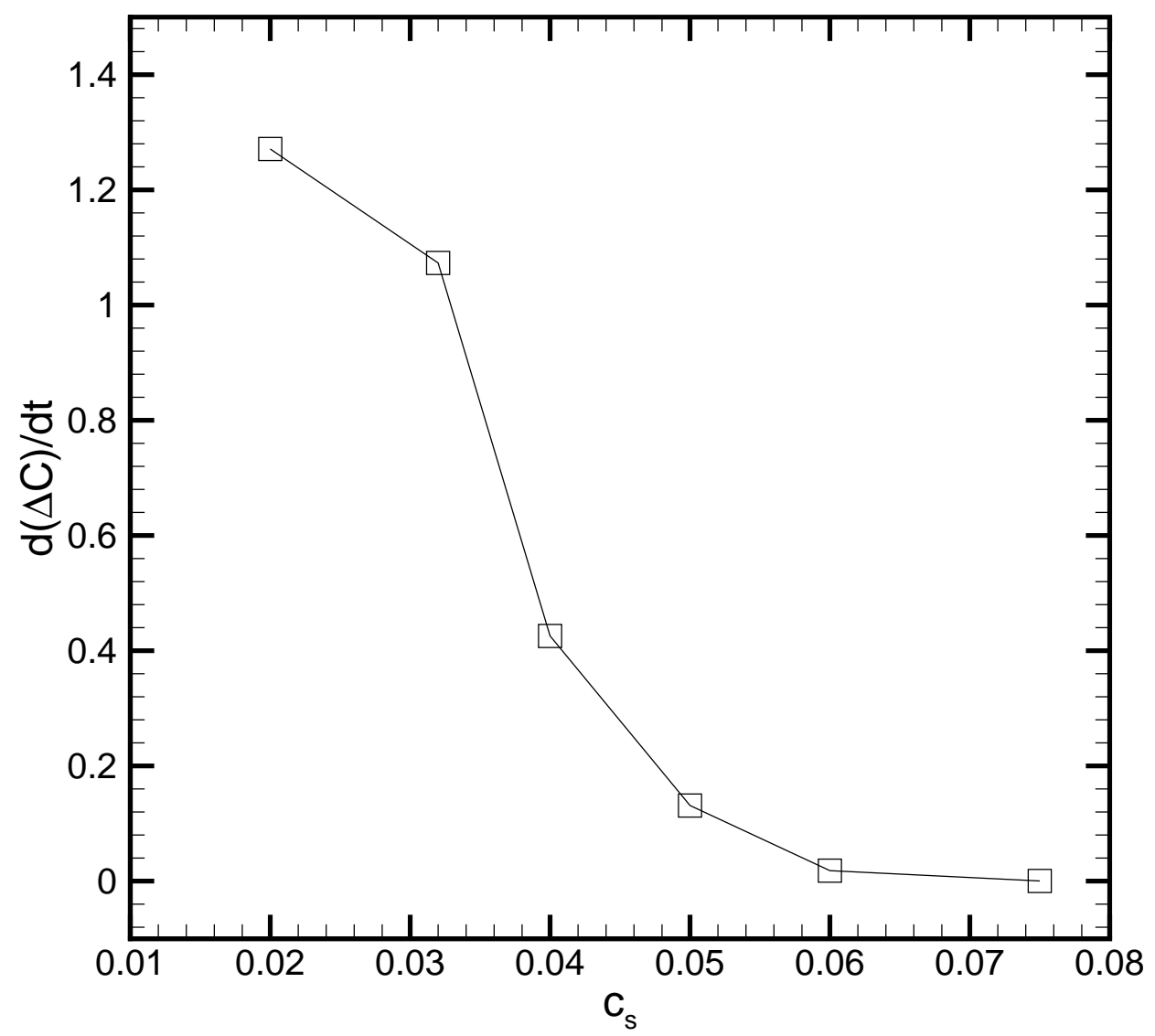

Figure 7.12: The slope of $\Delta C\left(c_{s}\right)$ before vortices emerge. This figure shows how the growth slope depends on the sound speed. It indicates that there are different sound speed regimes with a knee at $c_{s}=0.032$. Here, all runs had a planet mass $\mu=10^{-4}$. 


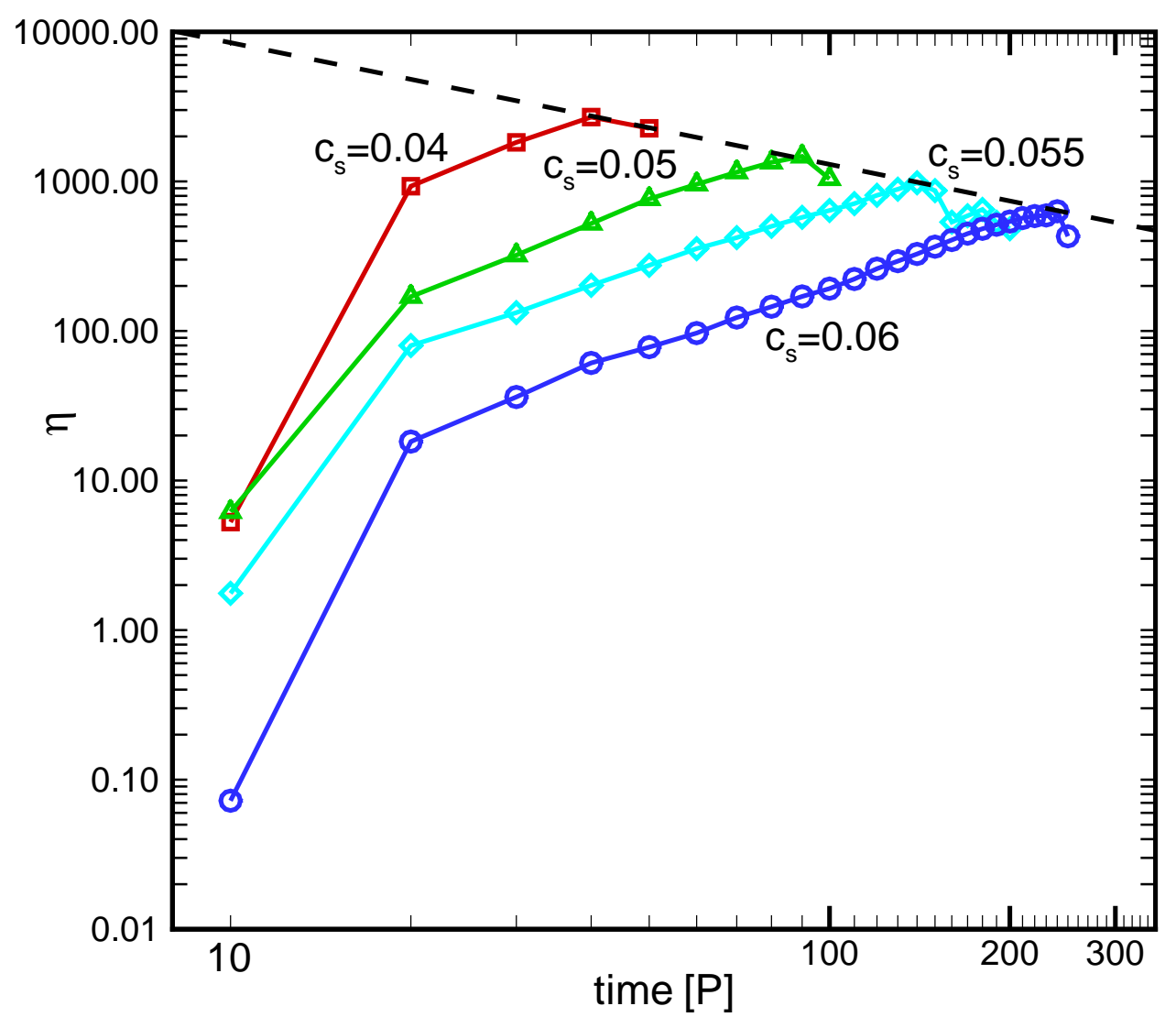

Figure 7.13: Evolution of $\eta$ (see Fig. 7.9 for a definition of $\eta$ ) for four intermediate sound speeds from $c_{s}=0.04$ to 0.06 . Here $\eta$ grows linearly after the disk has adjusted to the planet mass within 10-20 orbits. The dashed line indicates the empirical law for the threshold condition for $\eta_{t r}$. After the disk develops vortices, there's a distinct knee in $\eta$ marking the threshold condition. 


\section{Chapter 8}

\section{Particle Paths}

The disk responds to the planet's potential by creating spiral shock waves (Goldreich \& Tremaine, 1979) from dissipated waves launched by the planet. They deposit angular momentum in the disk. Such angular momentum exchange drives disk material away from the planet creating dips in the azimuthally averaged density distribution $\langle\Sigma\rangle$.

Fig. 8.1 compares the initial $\mathrm{PV}$ and density profiles with the profiles at $t=$ $50 P$. The potential vorticity shows two very distinct peaks $\Delta r \approx \pm 3.4 r_{H}$ and dips at $\Delta r \approx \pm 2.3 r_{H}$ developing from a constant initial profile. As described in $\S 6.2$, these potential vorticity dips develop eventually an instability, and consequently form vortices.

According to the conservation law of potential vorticity Eq. (6.14), $\zeta$ should be conserved along streamlines in an inviscid disk. In this case, however, streamlines 
of test particles show that for $|\Delta r|>1.3 r_{H}$ (see Fig. 8.2), the material passes through a spiral shock where the dissipation breaks the PV conservation. Fig. 8.2 also shows where the normal velocity component to the shock becomes supersonic. The shock starts at roughly $|\Delta r|>1.3 r_{H}$ for this particular sound speed $c_{s}=0.05$ indicated by the thick horizontal line. Since the inclination angle of the shock to the flow is quite large, the normal velocity component, is small. Only the normal velocity component shows a jump at the shock whereas the tangential component is smooth over the shock.

To establish the role of shocks in breaking the PV conservation, several runs with test particles are performed. By placing particles in regions where the potential vorticity change is the largest, i.e. between $|\Delta r|=1.5 r_{H}$ and $4 r_{H}$, we can follow the evolution of parameters like vorticity and PV along the stream lines.

Three particles from different regions are shown in Fig. 8.3. The blue curve shows $r(t)$ for a particle orbiting at $\Delta r=-2.5 r_{H}$ but, note, it crosses the shock at $\approx 2 r_{H}$. The deflection is due to the planets gravitational potential. The other two curves are for a particle at $-3.5 r_{H}$ (green) and $-4.95 r_{H}$ (red). The change of $r(t)$ near the planet has to be taken into account for explaining why the shock leads to a decrease in PV in some regions and to an increase in others. Also we note here that a particle at $-5 r_{H}$ passes through the shock almost twice compared to a fluid element at $-2.5 r_{H}$. That means, if the change of $\Delta \zeta$ would be the same for both locations, the growth rate for the particle at $r=-5 r_{H}$ would be twice as large. 
Fig. 8.4 describes how the potential vorticity changes which each pass through a shock. Each time a particle crosses the spiral shock, it gains or looses potential vorticity. Outside the shocked region, the potential vorticity of the particle stays constant (in agreement with the theory of potential vorticity conservation). Since the initial $\zeta$ was almost constant over the whole disk, the three particles start out with the same potential vorticity. However, due to the shock structure at the particular shock crossing point, the potential vorticity increases or decreases.

We can calculate the vorticity by taking the curl of velocity and find

$$
\omega=(\nabla \times \tilde{\boldsymbol{v}}) \cdot \hat{z}=\frac{1}{r} v_{\phi}+\frac{\partial v_{\phi}}{\partial r}-\frac{1}{r} \frac{\partial v_{r}}{\partial \phi}-2 \Omega
$$

Potential vorticity would be Eq. (8.1) divided by $\Sigma$ but we can neglect the density change here. Since the density is decreasing between $-5 r_{H}<\Delta r<5$ that would not explain why PV has increasing and decreasing values in the very same region (see Fig. 8.1). So, if we follow particles from that area, they will show a continuous decrease in density as in Fig. 8.5. Hence, the change in $\zeta$ also in $\omega$ can be due to only three parts which we define according to Eq. (8.1) as

$$
a_{1}=\frac{v_{\phi}}{r}, \quad a_{2}=\frac{\partial v_{\phi}}{\partial r}, \text { and } a_{3}=-\frac{\partial v_{r}}{\partial \phi}
$$

The contribution to $\Delta \zeta$ is shown in Fig. 8.6 which plots $a_{1}, a_{2}$, and $a_{3}$ as a function of time following a single Lagrangian particle. Only $a_{2}=\partial v_{\phi} / \partial r$ shows 
an increase when the particle moves through the shock. The other values return after the shock to the pre-shock value. The sum of $a_{1}+a_{2}+a_{3}$ is shown in Fig. 8.4 as the green curve.

We can also zoom into the shock structure and it reveals that a standard step like function is not adequate to describe this shock. Fig. 8.7 shows that before the particle hits the shock, it experiences a density decrease. The shock itself has a density increase of post-shock over pre-shock density $\Sigma_{2} / \Sigma_{1}=1.7$. However, the shock is followed immediately by a rarefaction wave and then an increase in density back to almost the same pre-density value. That raises the question where the pre and post-shock region starts and ends. In a mathematical shock (i.e., a step-like function) the question is not relevant since the pre and post-shock values are constant. However, in our case with a quite complicated shock, the question is if one should take the asymptotic values far away from the shock or is it better to use the minima and maxima along the particle path. Since the asymptotic densities are almost equal for pre and post-shock, the Rankine-Hugoniot relations (Laney, 1998) $\Sigma_{1} v_{1}=\Sigma_{2} v_{2}$ would provide no shock at all. Hence, a better choice is taking the minima and maxima from the particle streams for matching the Rankine-Hugoniot relations with the shock conditions. 


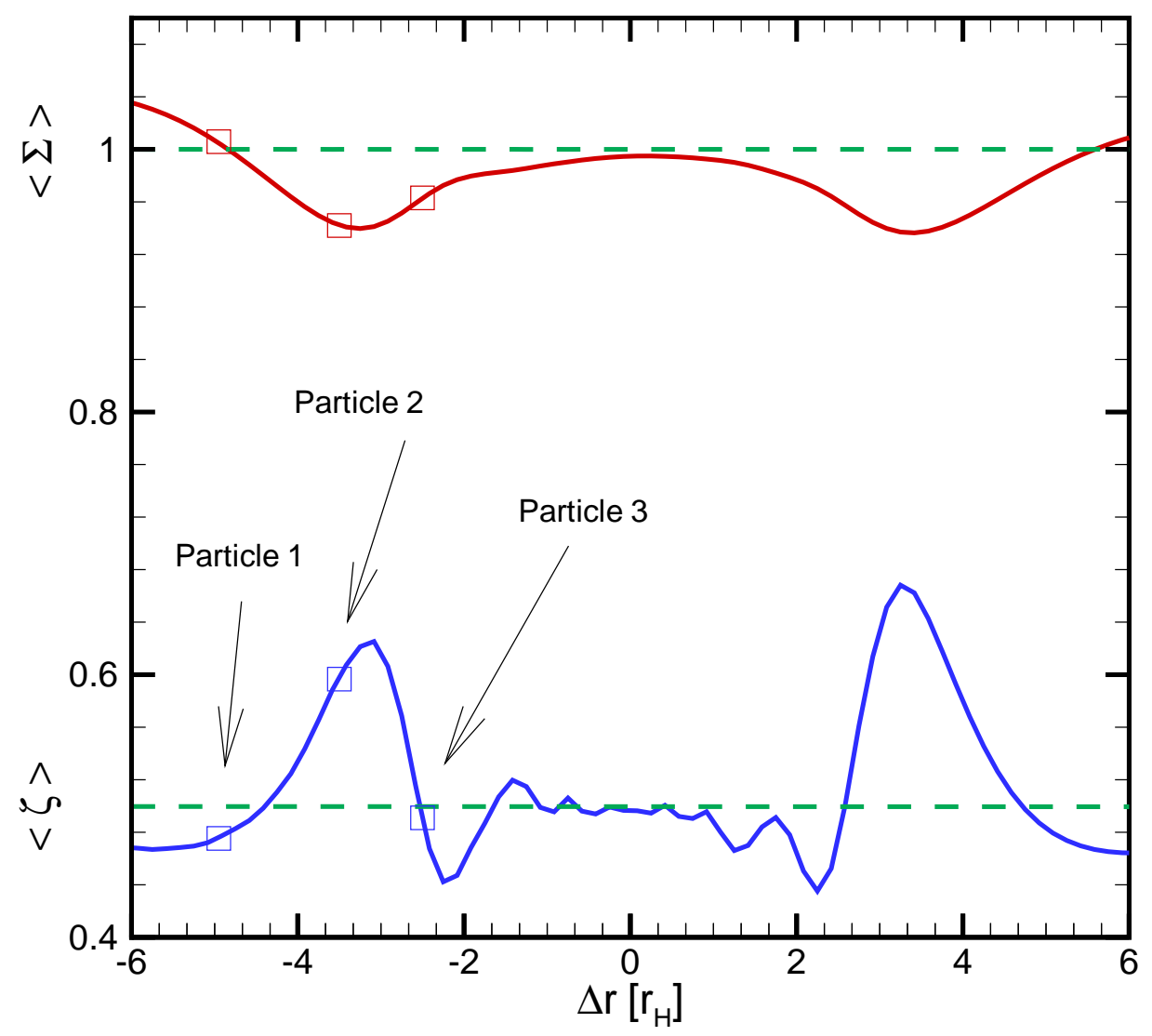

Figure 8.1: Azimuthally averaged surface density $\langle\Sigma\rangle$ and potential vorticity $\langle\zeta\rangle$ at $t=50 P$ for the sound speed $c_{s}=0.05$. The dashed lines represent the initial values for the surface density and the potential vorticity. In this plot the density is reduced to a flat profile to enhance the features. The simulations were done with an actual density profile of $\Sigma \propto r^{-3 / 2}$. The squares denote the location of test particles and where they lie on the potential vorticity and density curve. 


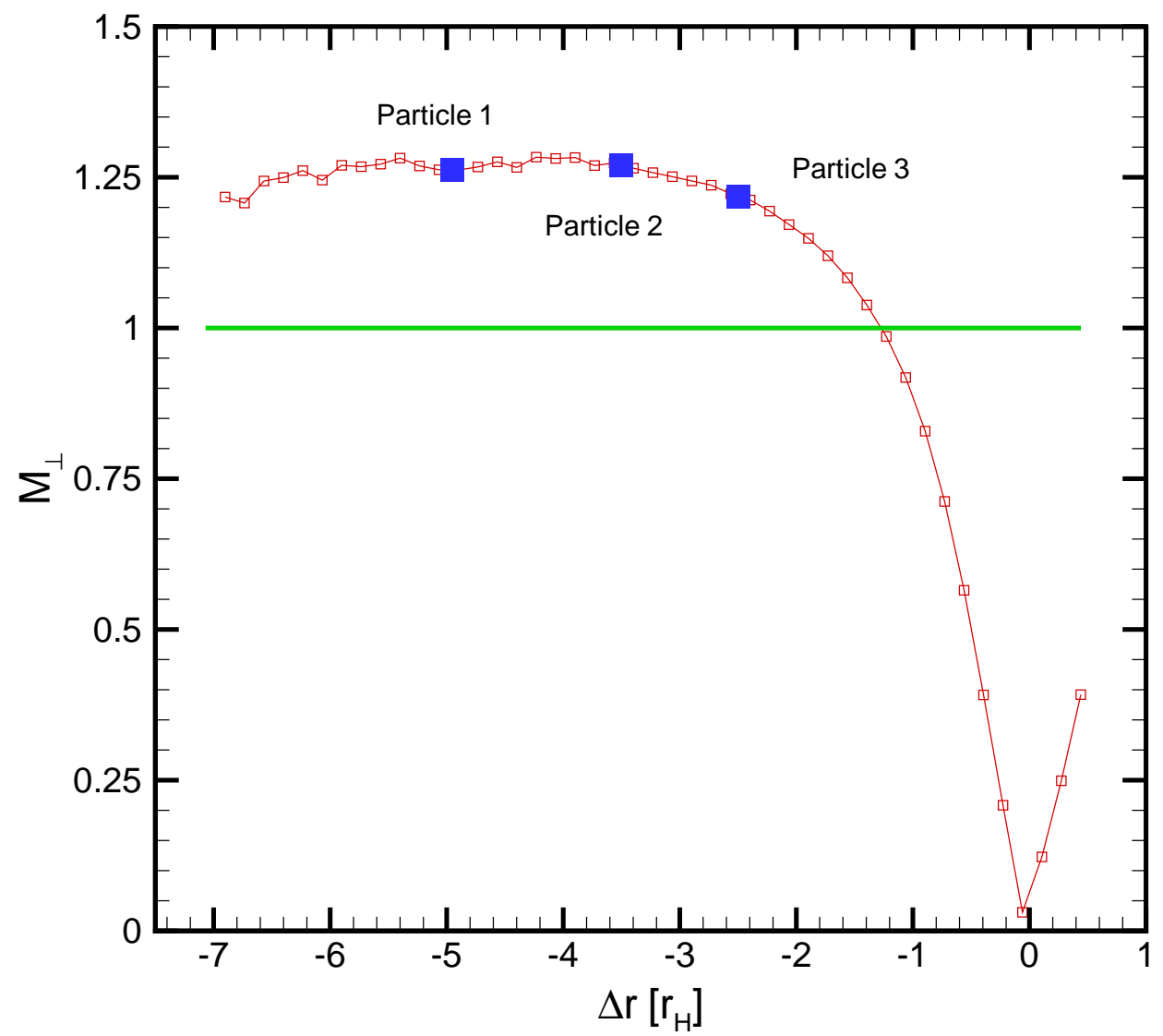

Figure 8.2: Shock structure in terms of Mach number. Taking into account the obliqueness of the shock, the Mach number $M_{\perp}$ of this shock is very small. The supersonic region starts around $-1.2 r_{H}$ and never exceeds $M_{\perp}=1.3$. The thick line denotes the supersonic regime where $M_{\perp}>1$. This figure is based on a simulation with $c_{s}=0.05$ and $\mu=10^{-4}$. The blue squares denote the radial location of the test particles in this section. 


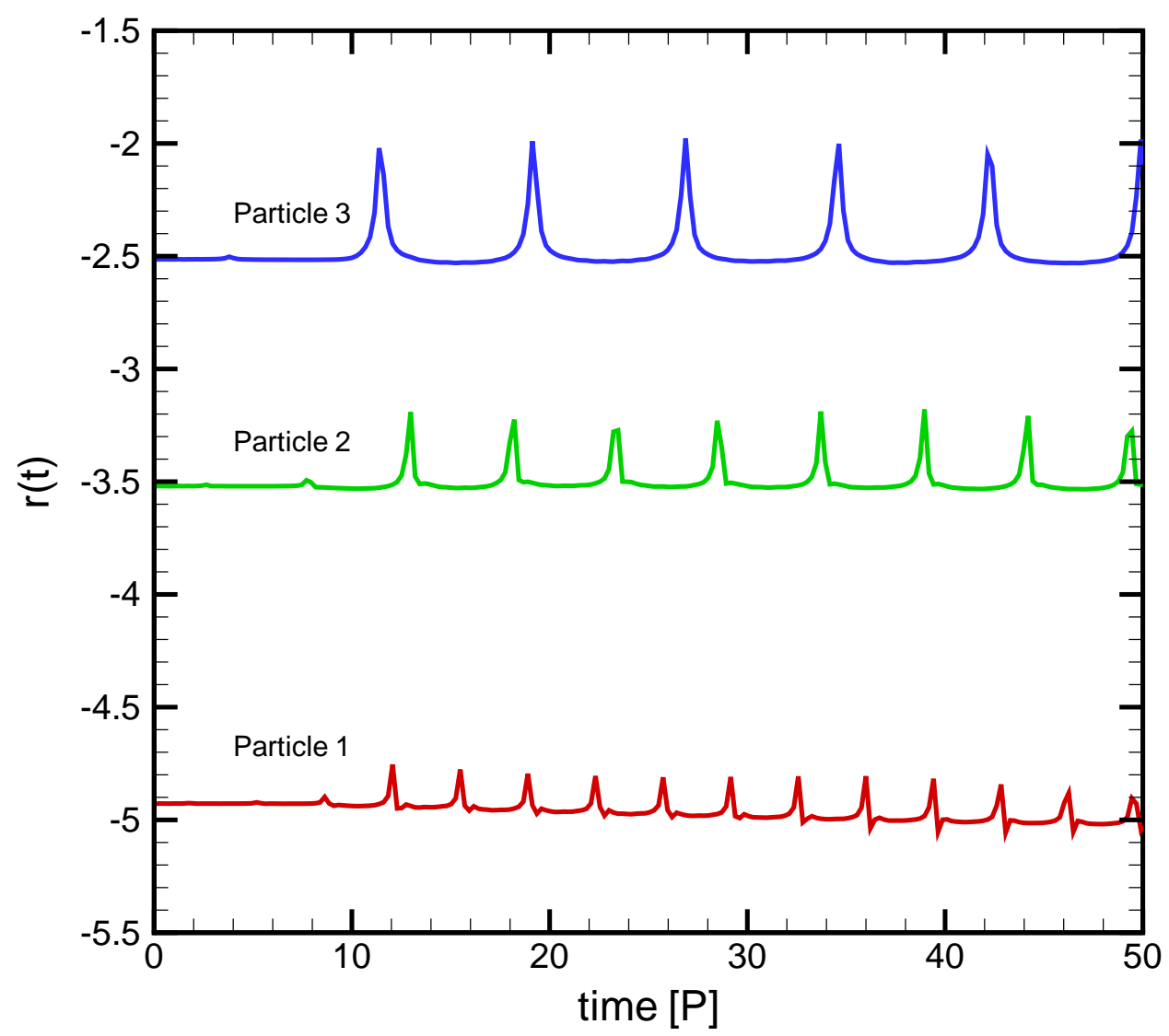

Figure 8.3: The radial component of several particles in the separatrix initially at $r_{0}=-2.5 r_{H}$ (blue; particle 3 ), $r_{0}=-3.5 r_{H}$ (green; particle 2), and at $r_{0}=$ $-4.95 r_{H}$ (red; particle 1). After the planet mass has been fully turned-on, spiral shocks are forming, and particles are passing through the shocked regions. Fluid elements do not only experience a change in the normal velocity component but also see an attraction from the planet when they are close. Overall, the particle does not change its orbital $r(t)$ by much. After the shock it returns to almost the same $r(t)$ as before the shock. Except, the red line shows a slight decrease within 50 orbits which means it moves further away from the particle. This is understandable since the overall density decreases in that region. 


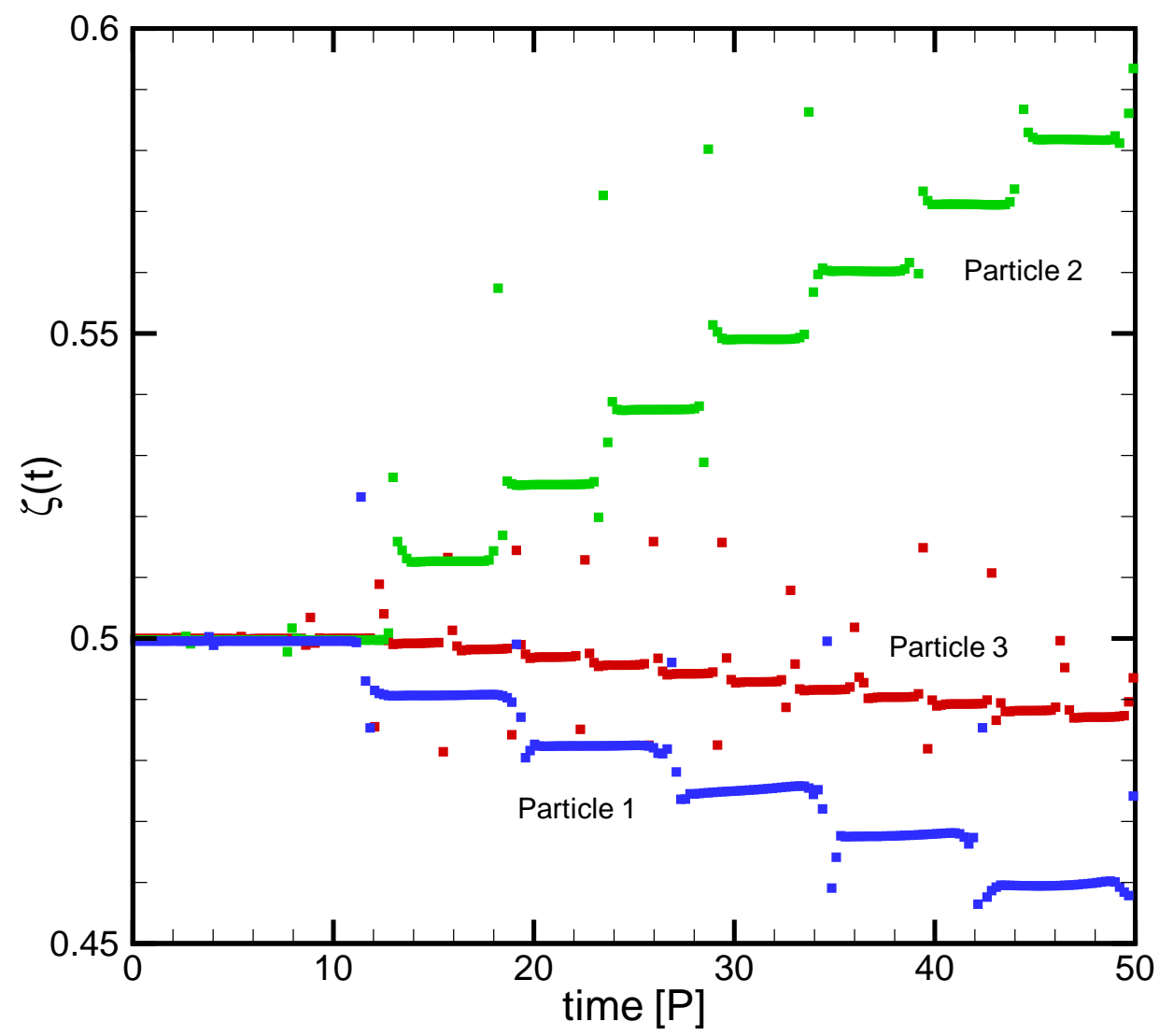

Figure 8.4: Potential vorticity evolution along stream lines. Three particles are crossing the spiral shock at different location But they almost return to their previous orbits (see Fig. 8.3) at $r_{0}=-2.5 r_{H}$ (blue; particle 3), $r_{0}=-3.5 r_{H}$ (green; particle 2), and at $r_{0}=4.95 r_{H}$ (red; particle 1). However, the shock's dissipative mechanism changes the particles potential vorticity. After the particle comes out of the shock, it has a different PV value than before. After the shock, the PV value stays almost constant. Time averaged values are plotted here with a time window of $0.5 P$. 


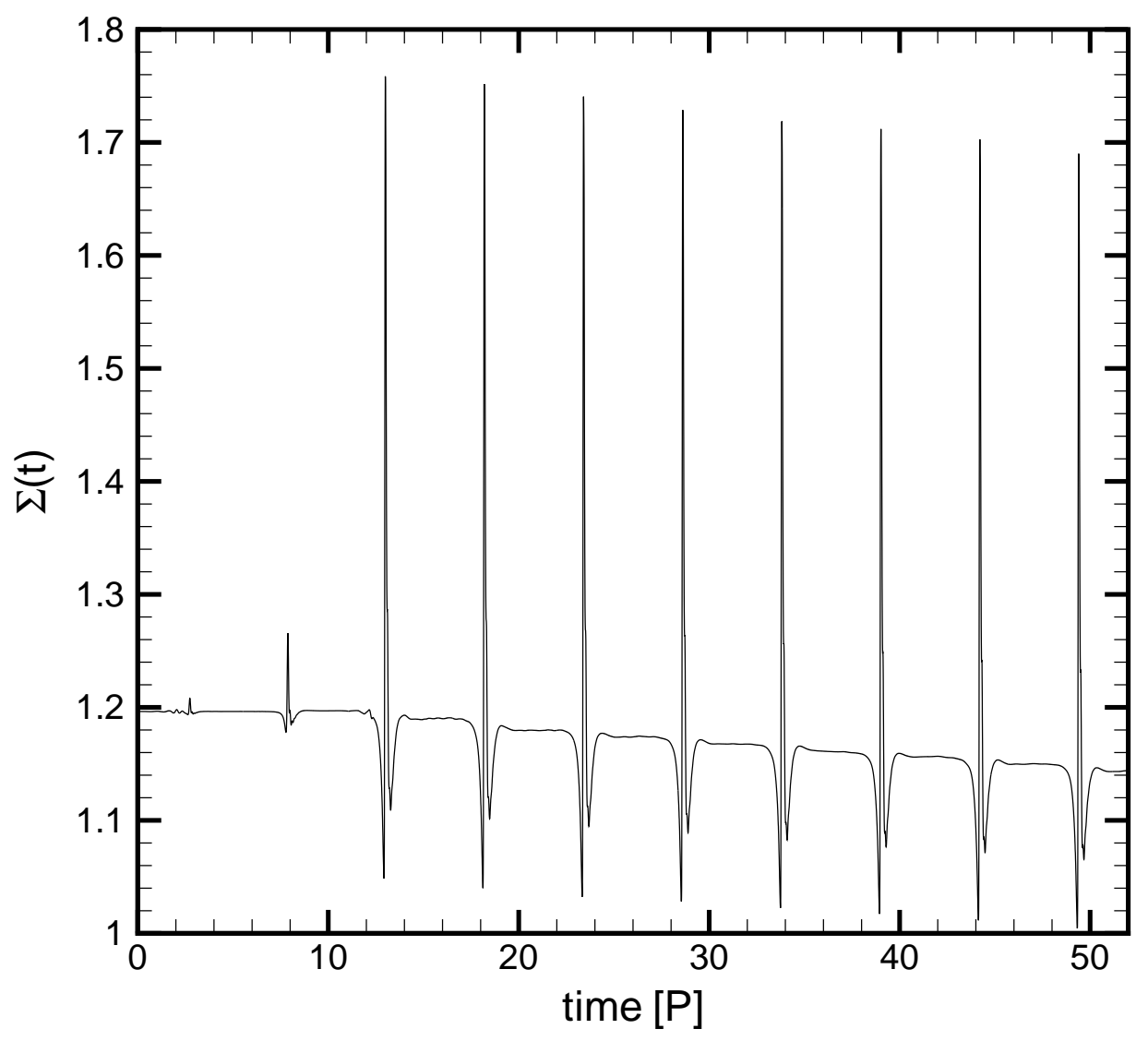

Figure 8.5: Density evolution of a particle in the separatrix region. Following a Lagrangian particle (here particle 2), the density decrease is continuous except when it passes through a shock. All particles within $\pm 5 r_{H}$ show a similar decrease like the one shown here. 


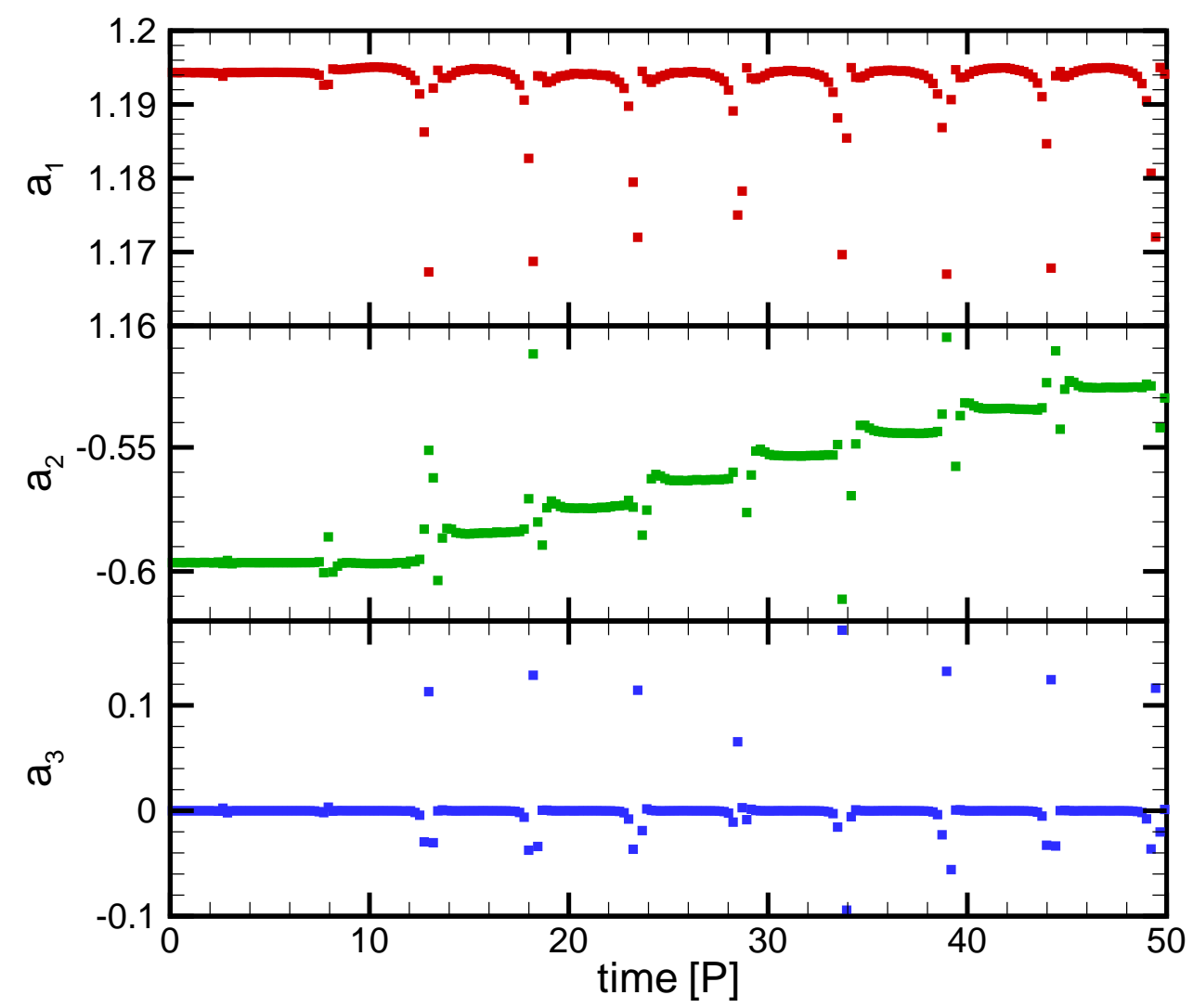

Figure 8.6: Dividing the vorticity into its three contributions $a_{1}, a_{2}$, and $a_{3}$. As we see only $a_{2}$ shows a step-like function. The others are returning to their pre-shock values. Hence, we can conclude the change in vorticity is largely due to the change in radial gradient of the angular velocity $v_{\phi}$. Only the values for particle 2 are plotted here. Others are similar. The minimum at $t=23.35 P$ and the maximum at $t=23.4 P$ were used for vorticity jump calculations (see $\S 9$ ). 


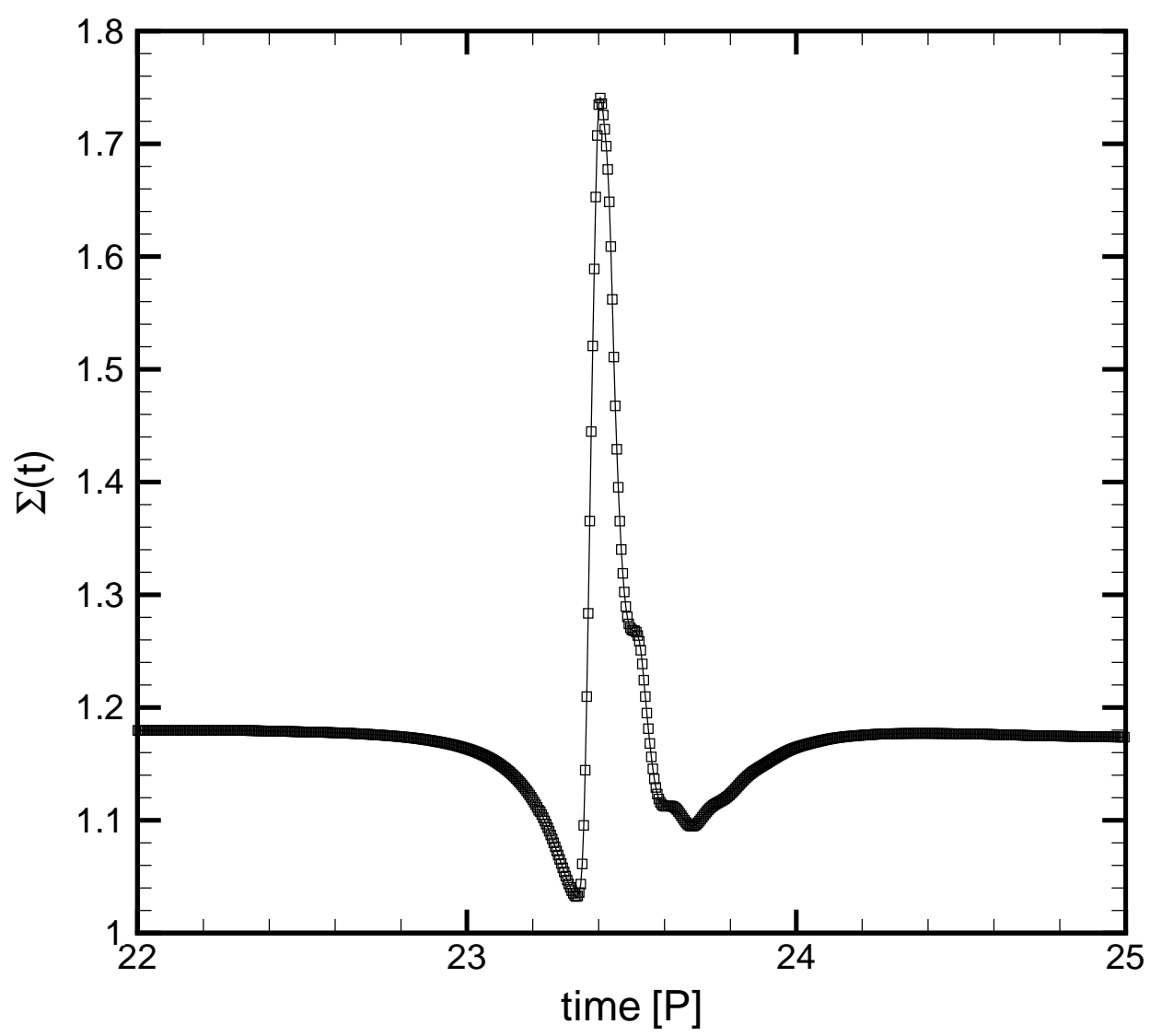

Figure 8.7: Same as Fig. 8.5 but zoomed in for getting a better look at the shock structure. Before the particle hits the shock, it experiences a significant density decrease. The shock density itself jumps by a factor of 1.7. However, the shock is immediately followed by a rarefaction wave and a subsequent increase to an almost pre-shock density. 


\section{Chapter 9}

\section{Shock Structure and PV Jumps}

\section{$9.1 \quad$ Shock Structure}

As we discussed in $\S 6.2$, the spiral shocks are responsible for destroying the conservation of potential vorticity (PV) since they are equivalent of a strong viscous stress in the momentum equation. Taking the curl of the two-dimensional momentum equation including a viscous stress, the Lagrangian derivative will be

$$
\frac{D(\zeta \hat{z})}{D t} \neq 0
$$

where the flow is dissipative.

The question is, how does the shock structure affect the amount of dissipation and consequently the change in $\zeta$. An expression for the vorticity jump across a shock was first obtained by Truesdell (1952) where he considered the vorticity 
jump in a tangential direction across a two-dimensional steady shock in uniform flow. The result by Truesdell (1952) was later independently re-derived by Lighthill (1957). He generalized the case to three-dimensional shocks and expressed Truesdell's vorticity jump relation in term of the axes of principal curvature of the shock. Hayes (1957) derived the vorticity jump using only conservation of mass and momentum and generalized it from a steady shock in uniform flow to the general case of an unsteady shock moving into a non-uniform flow. Kornreich \& Scalo (2000) describe how the change in vorticity (and then also potential vorticity $\Delta \zeta$ ) can be expressed as a function of non-uniformities in the flow ahead of the shock. The paper by Kevlahan (1997) shows that this mechanism is almost always neglected in discussions of vorticity generation across a shock. He derives a simple relation for the vorticity jump $\Delta \omega$. Using his fomula and by neglecting the baroclinic term, we find

$$
\Delta \omega=-\frac{\delta^{2}}{1+\delta} \frac{\partial u_{\perp}}{\partial \tau}
$$

where $\delta=\Sigma_{2} / \Sigma_{1}-1$ with the subscript $\Sigma_{1}$ denoting the density in the pre-shock region and $\Sigma_{2}$ the density in the post-shock region. $u_{\perp}$ is the normal component of the flow ahead of the shock relative to the shock speed and $\partial / \partial \tau$ is the tangential derivative. Since $\delta+1=\Sigma_{2} / \Sigma_{1}=M_{\perp}^{2}$, the equation above can be rewritten as potential vorticity

$$
\Delta \zeta=-\frac{\left(M_{\perp}^{2}-1\right)^{2}}{M_{\perp}^{2}} \frac{1}{\Sigma_{2}} \frac{\partial u_{\perp}}{\partial \tau} .
$$


To study this equation and to compare its prediction with results from the simulation, we have to define the shock and pre-shock locations. As we see in Fig. 8.7, the flow goes through a reduced density region before it hits the shock. The pre-shock region is defined as the minimum of density just before the shock. The density maximum is set to be at the shock location. Fig. 9.1 depicts four flow lines going through the shock. Eq. (9.3) asks for the velocity gradient in the pre-shock region which we see in Fig. 9.2 in terms of perpendicular Mach number. For this figure we have to find out the inclination angle of the flow with the shock. By defining the shock location as the location of maximum density, and with the velocity vectors in the pre-shock region, we can determine the inclination angle as shown in Fig. 9.3.

Since also the pre-shock density plays a role in Eq. (9.3), we can study that with Fig. 9.4. We see a distinct density minimum in the pre-shock region at $\Delta r \approx-3 r_{H}$ for $c_{s}=0.05$. Next, we look at the tangential gradient of the normal component of the flow ahead of the shock. This is done in Fig. 9.5 and 9.6.

A further complication arises with the attractive potential of the planet: A particle on a streaming orbit approaching the planet will fall by some amount toward the planet before hitting the shock (Fig. 9.1). Usually a test particle in a streaming orbit does not change $r(t)$ much. Only in the vicinity of the planet, the time average over one period $\langle r(t)\rangle$ is different from the current $r(t)$. After the shock, the particle goes back to almost the same orbital $r(t)$ as before the shock. At 
the shock itself, the particle is deflected due to the change in the normal component of the velocity. The change in vorticity is due to the shock conditions where the particles goes through the shock. Therefore, the change in PV is reflected by the location of the shock crossing $r_{s}$ and not by the azimuthally averaged radial orbit $\langle r(t)\rangle$, where the particle with the new PV value spends most of the time. For example, particle \#4 in Fig. 9.1 orbits at $\Delta r=-2 r_{H}$. However, the change in PV is due the shock crossing at $\Delta r_{s} \approx-1.3 r_{H}$. Fig. 9.7 shows the correlation between the orbit $\langle r(t)\rangle$ away from the planet and the shock crossing location $\Delta r_{s}$.

Since we want to figure out where the inflection points in the PV profile (Fig. 9.8) come from, we take everything from Eq. (9.3) into account and calculate what the jump $\Delta \zeta$ would be according to the shock condition. Fig. 9.9 shows the comparison of theory versus what actual test particles experience when they pass through the shock. Once the shift in $\Delta r$ is applied, the overall fit with Eq. (9.3) is better than a factor of two for most measurements. It is probably possible to obtain a better fit with a better matching polynomial compared to Fig. 9.5. This is true for the regions III and II (see Fig. 9.8). However, region I is just where the tip of the shock starts. This region is heavily affected by the secondary potential of the planet and the question is if the simple analytical estimate of Eq. (9.3) actually applies to this region. In a non-shocked region Eq. (9.3) predicts that $\Delta \zeta$ would be zero. However, wee see a decrease in potential vorticity right at the tip of the shock (region I). This region eventually becomes unstable 
and develops vortices as seen in Fig. 6.4. According to Li et al. (2001), regions II and III should eventually become unstable, too, but even our longest simulations did not show vortices developing there. This might be due to the shallower dip and peak in these regions. See also $§ 11$.

As it turns out, the dip in the PV profile in region I is difficult to explain and its physics difficult to pin down. It is similar to a boundary layer problem with a Kelvin Helmholtz instability. As described in Chapter 11, an increase in resolution leads to a decrease in region I, which means the scale length of the boundary layer also decreased. 


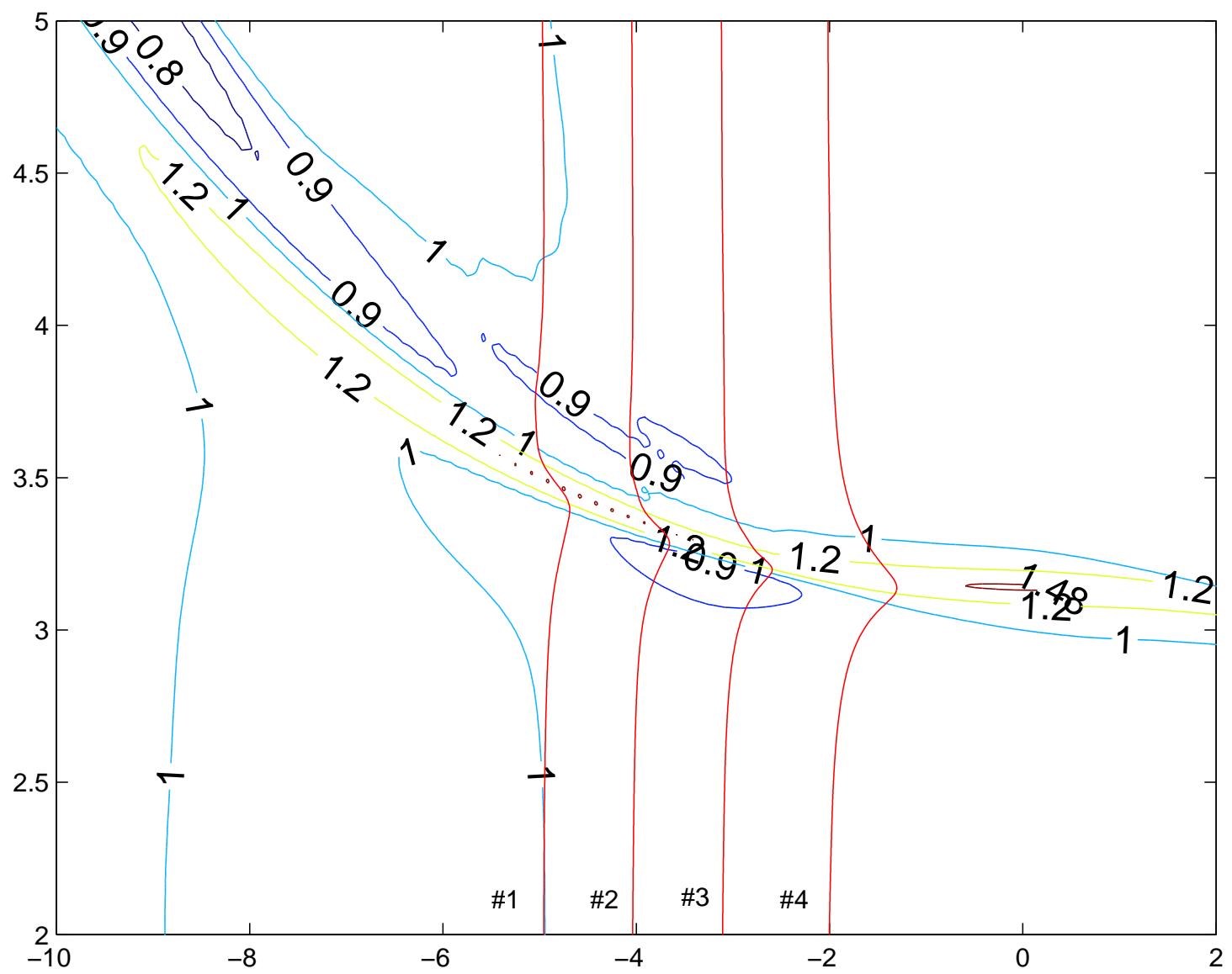

Figure 9.1: Four streamlines and density contours. The four red lines denote Lagrangian stream lines in the inner disk passing through the shock. They are not only influenced and deflected by the shock, they are also attracted by the planet located at $r=0$ and $\phi=\pi$. The contours show the shock and the density minima in the pre-shock region (see also Fig. 9.4). Here particles move upwards. 


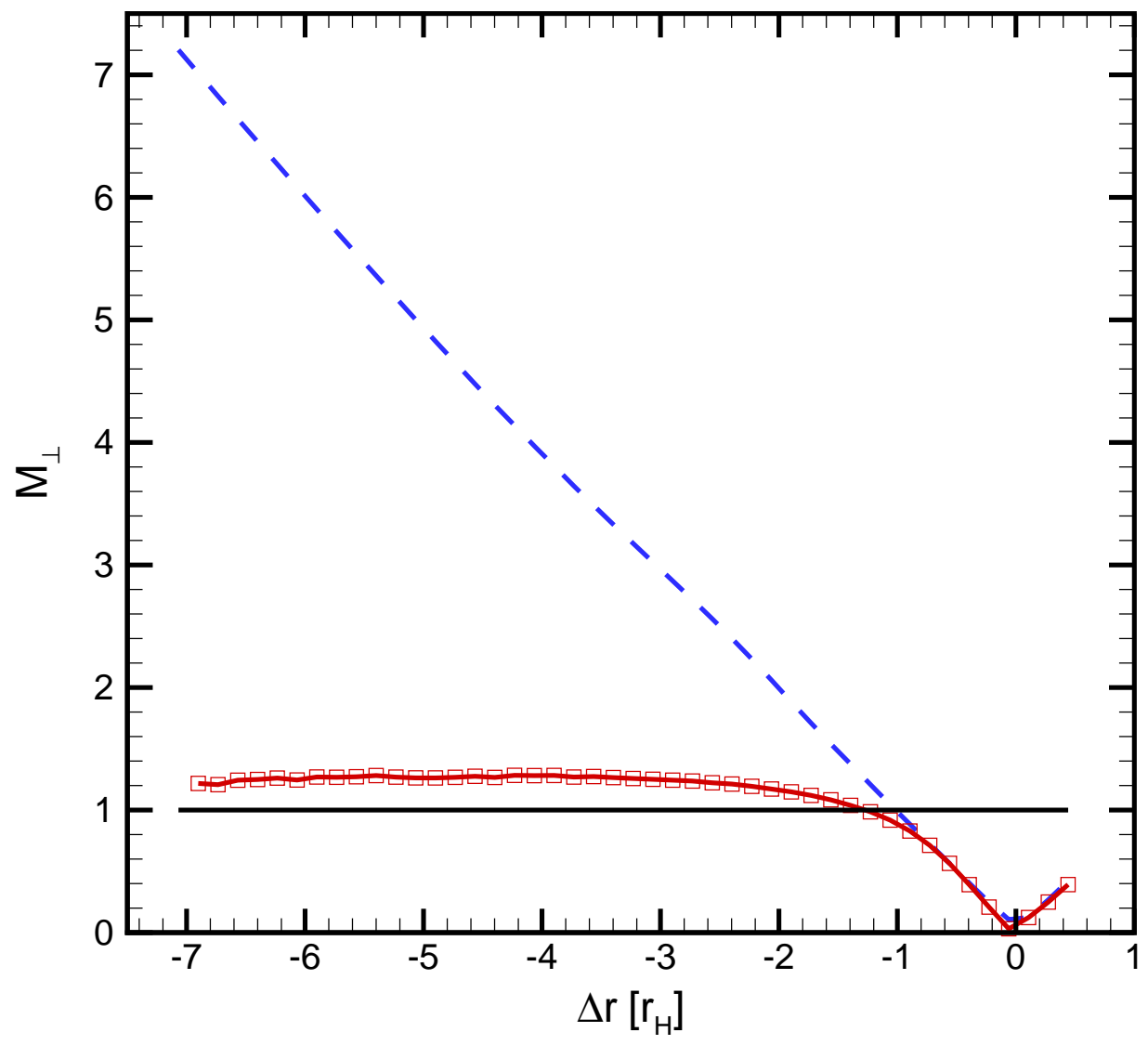

Figure 9.2: Shock structure in terms of Mach number. Taking into account the obliqueness of the shock and the increasing relative velocity (dashed line) to the shock front, the Mach number $M_{\perp}$ of this shock is very small. The supersonic region starts at around $-1.2 r_{H}$ and never exceed the $M_{\perp}=1.3$. The thick line denotes the supersonic regime where $M_{\perp}>1$. This figure is based on a simulation with $c_{s}=0.05$ and $\mu=10^{-4}$. 


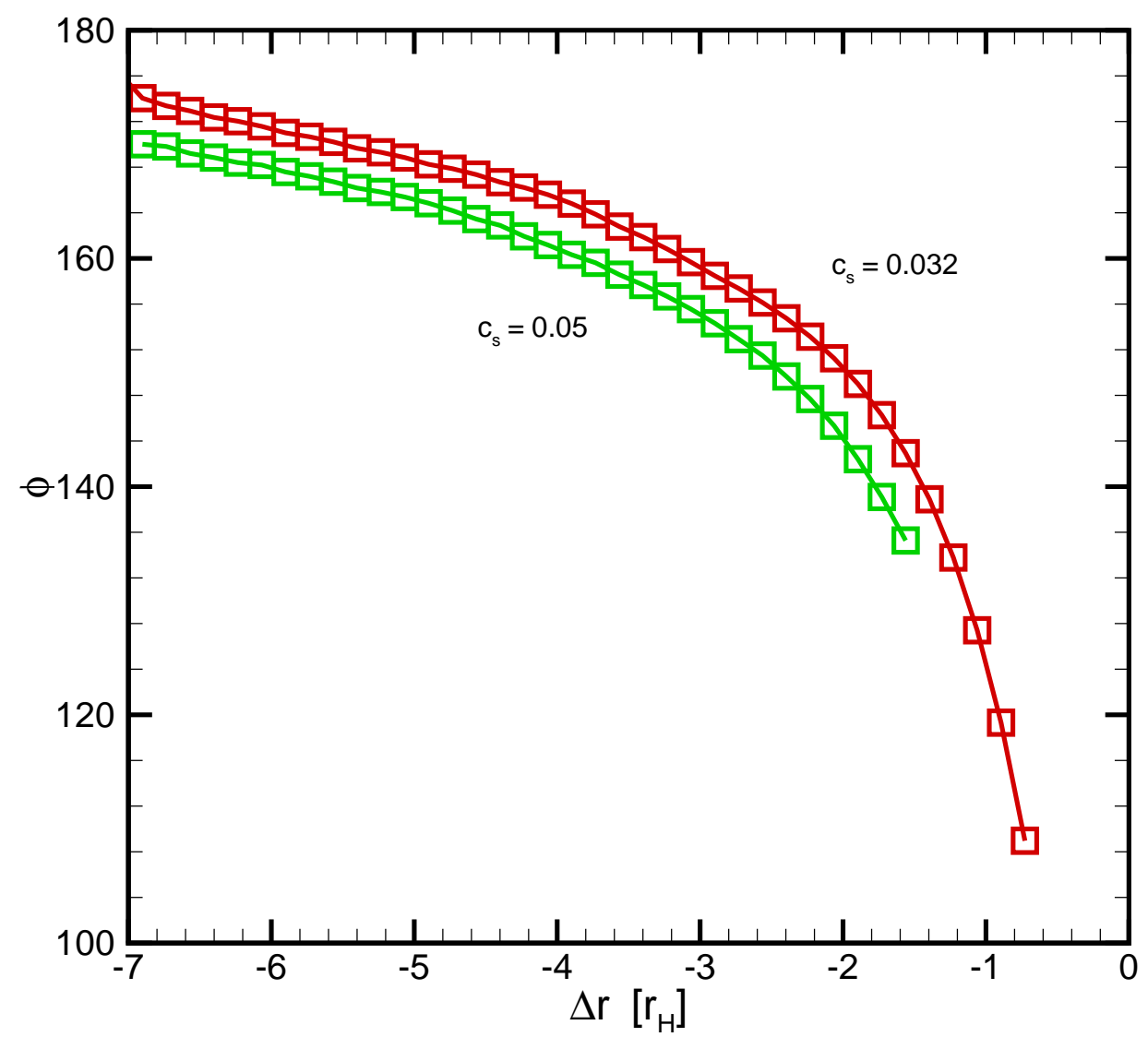

Figure 9.3: Inclination of the disk flow with the shock for $c_{s}=0.032$ (red) and $c_{s}=0.05$ (green). Due to the spiral nature of the shock, the flow passes through the shock with a strong shear flow, i.e. $\approx 170^{\circ}$ at $\Delta r \approx-7 r_{H}$. Streamlines closer to the planet have a more head-on collision with the shock. Squares denote the grid resolution of $(300 \mathrm{x} 1200)$ in this run from an interpolated shock front position. 


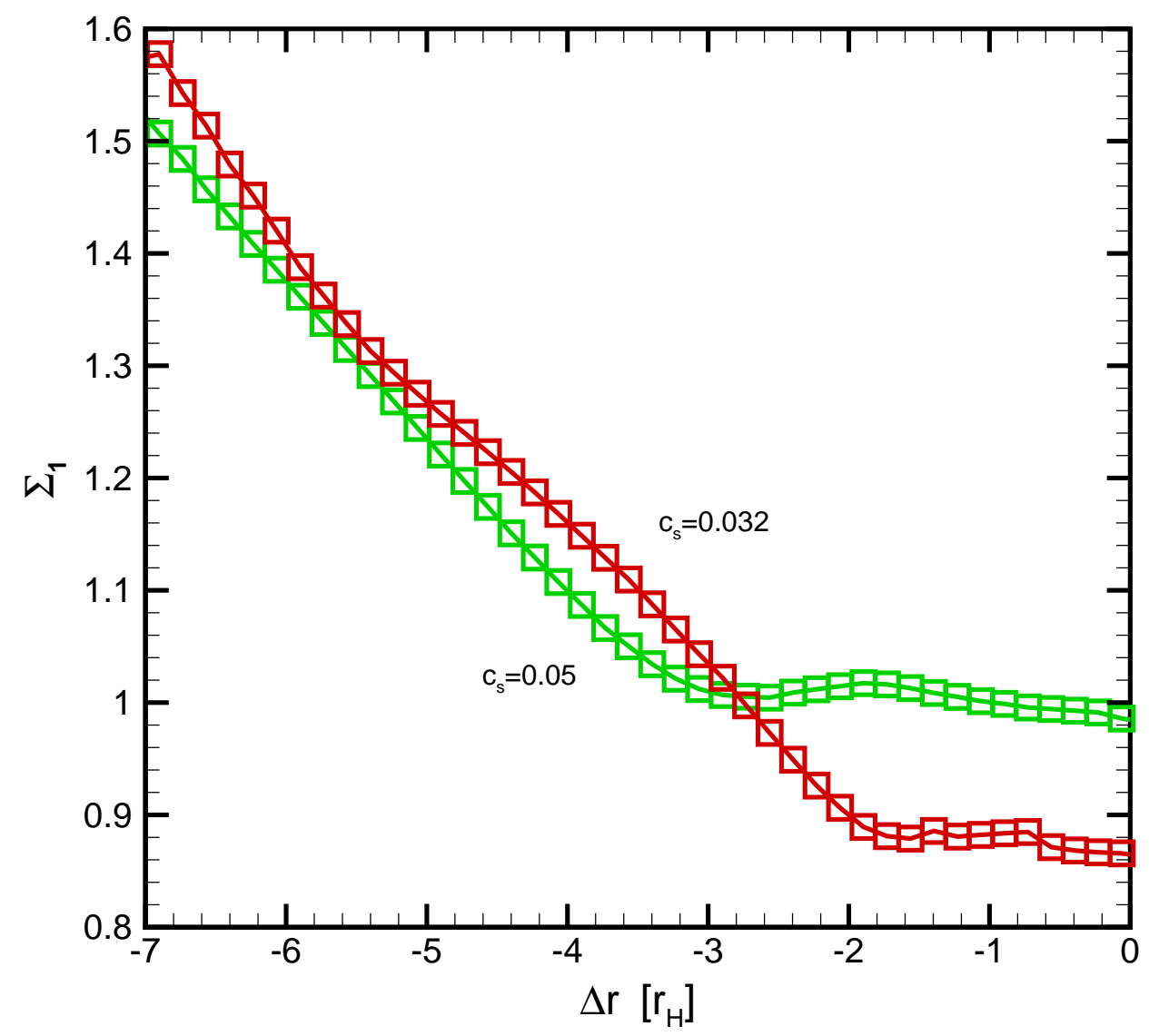

Figure 9.4: Density $\Sigma_{1}$ in the pre-shock region showing a distinct minimum at $\Delta r \approx-3 r_{H}$ for $c_{s}=0.05$ (green) and a mimimum at $\Delta r \approx-1.6 r_{H}$ for $c_{s}=0.032$ (red). 


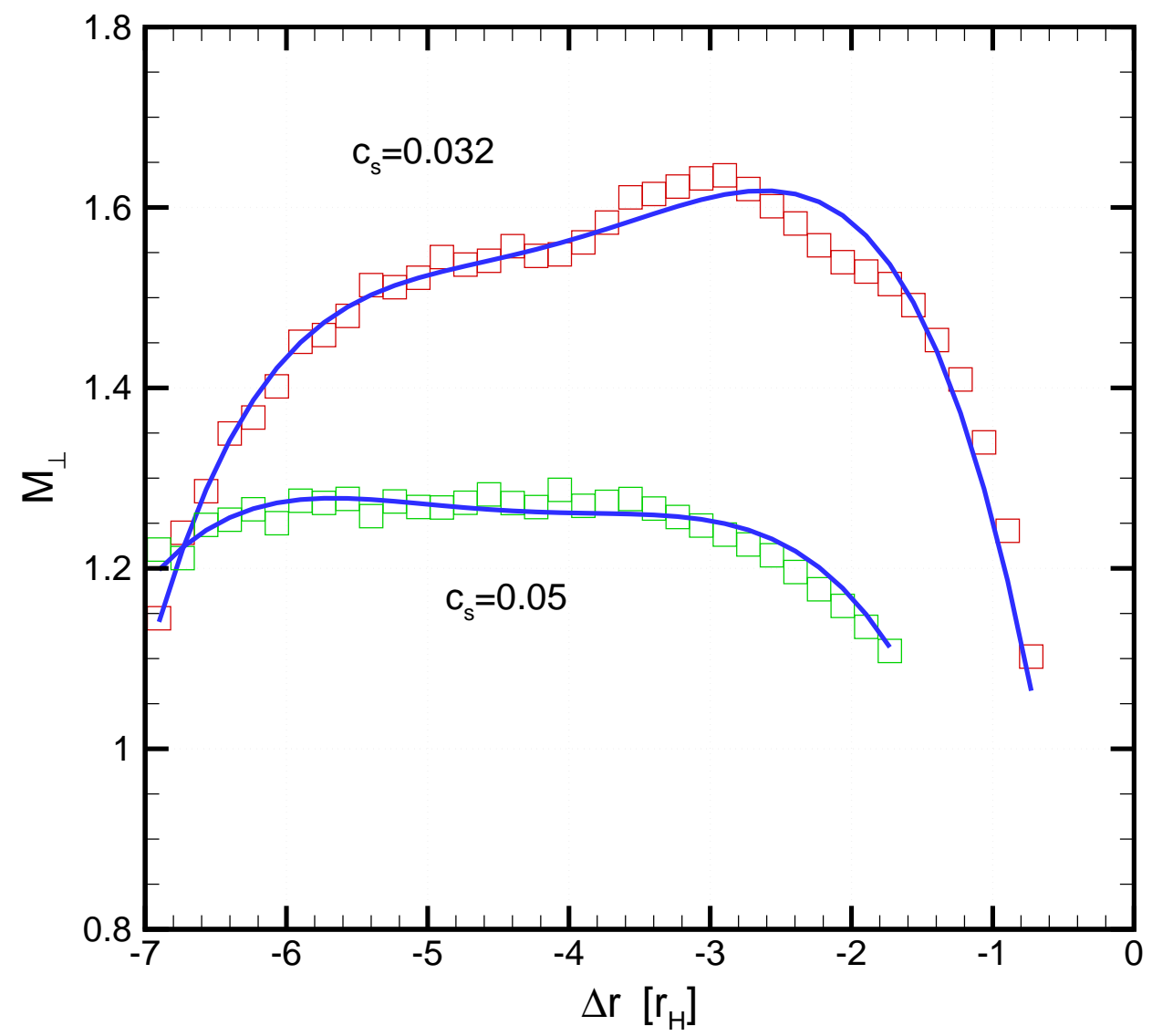

Figure 9.5: Shock structure in terms of Mach number for two different sound speeds $c_{s}=0.032$ (red) and $c_{s}=0.05$ (green), similar to Fig. 9.2 but taking into account the obliqueness of the shock and the increasing relative velocity. The symbols stop where the shock ends before it reaches the planet at $\Delta r=0$. 


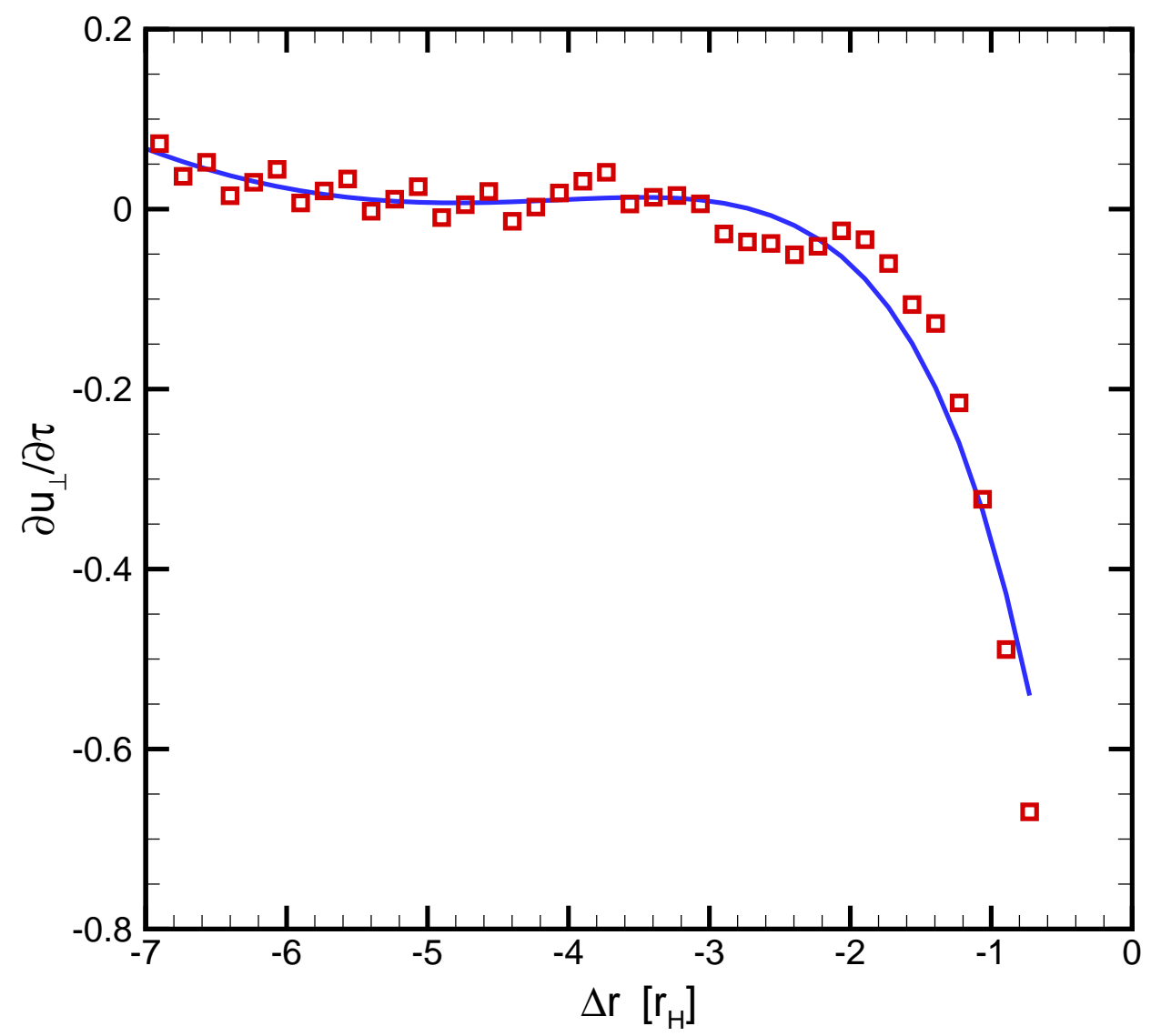

Figure 9.6: Gradient of the shock Mach number for sound speed $c_{s}=0.032$. The blue line denotes the gradient from a polynomial fit to the shock structure in Fig. 9.5. 


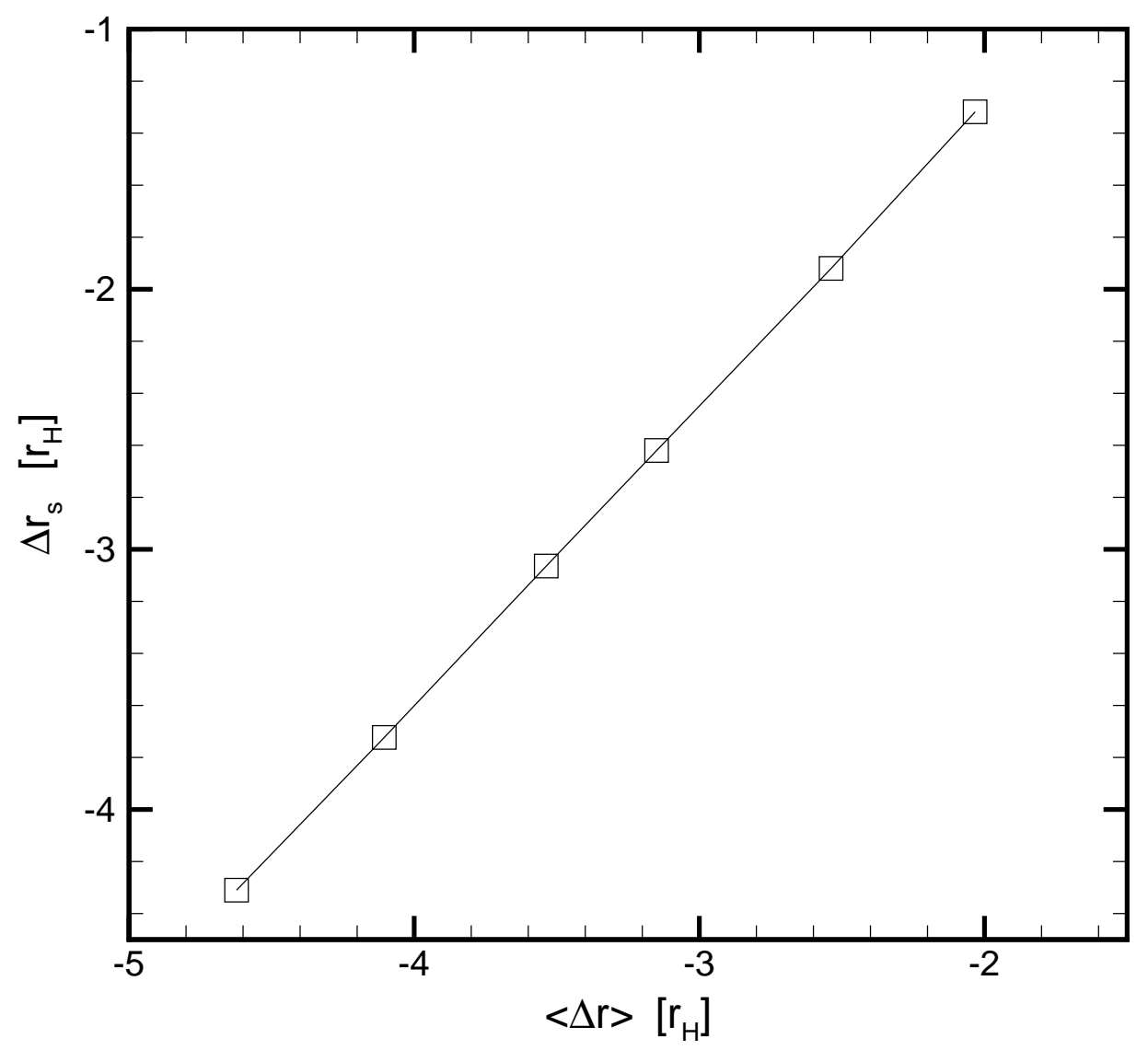

Figure 9.7: Change of orbit due to the planet. A particle spends most of its time at a constant $r(t)$. When it comes close to the planet, it falls towards it and crosses the spiral shock at a shifted location. For example, a particle usually at $\Delta r=-2 r_{H}$ passes through the shock at $\Delta r_{s}=-1.3 r_{H}$. Therefore, it was shifted by $0.7 r_{H}$ due to the planet's potential. 


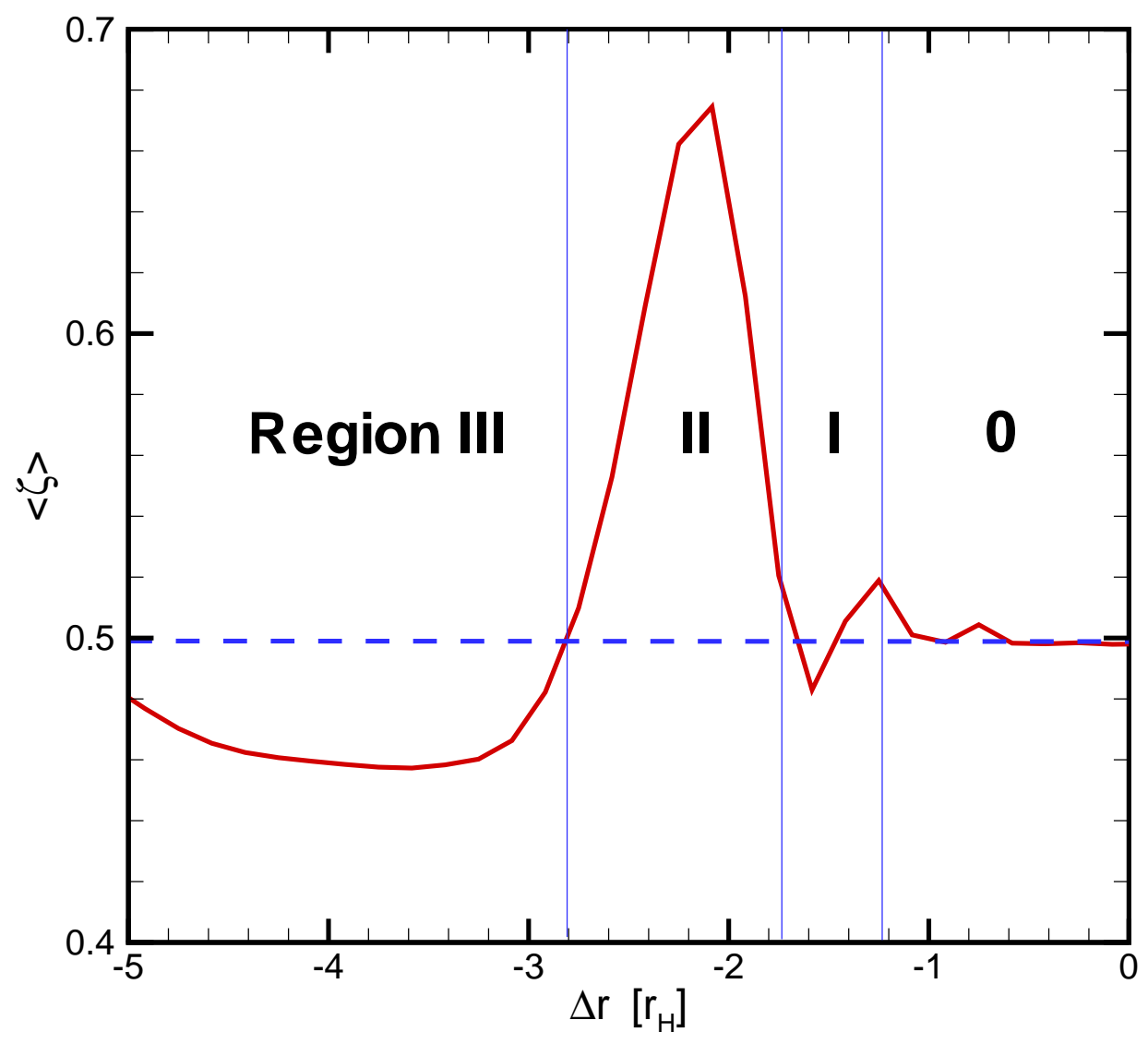

Figure 9.8: Measured azimuthally averaged PV for $c_{S}=0.032$. The dashed line shows the initial potential vorticity, the solid line is $\langle\zeta\rangle$ at $t=20 P$. Compare this figure with Fig. 9.9 and see that the region where vorticity is lost or gained match very well except in Region I. Regions are numbered where we see large PV changes. 


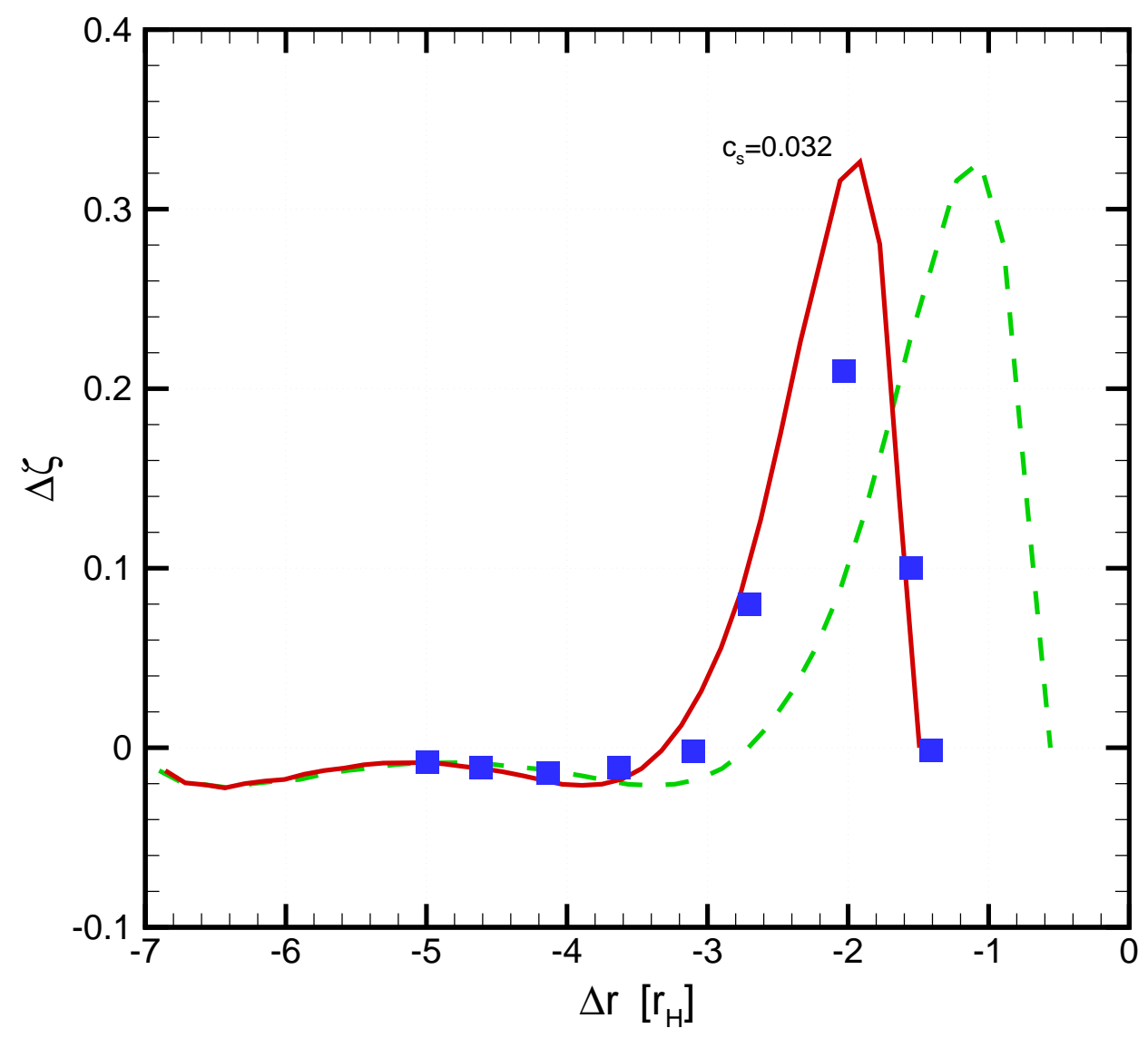

Figure 9.9: Expected change of PV with each shock passage for $c_{s}=0.032$. The dashed line is without considering the attraction by the planet. The red curve plots the change of potential vorticity at the radius $\Delta r$ where the particle spends most of its time. The blue squares denote the actual measurement of $\Delta \zeta$ of test particles. 


\section{Chapter 10}

\section{Some Multi Planet Simulations}

Among the discovered planets (see Marcy et al. (2000) and also http://www.obspm.fr/encycl/encycl.html by J. Schneider for an up-to-date list) the number of multiple planets is rising. As of March 2004, a total of 13 multiple planet system were among a total of 105 extrasolar planetary systems. Hence, the time evolution of a disk with two embedded protoplanets is studied here. The gravitational potential in Eq. (2.14) is extended by an additional term

$$
\Phi_{2}=-\frac{G M_{2}}{\left(r^{2}+r_{2}^{2}-2 r r_{2} \cos \left(\phi-\phi_{2}\right)+s_{2}^{2}\right)^{1 / 2}} .
$$

The second planet is located at $r_{2}=1.32$ with a 10 Roche radii separation from

the first planet. Both planet masses were defined as $\mu=10^{-4}$. The following simulations have a global sound speed $c_{s}=0.04$ and a density profile of $\Sigma(r) \propto$ 
$r^{-3 / 2}$ which has been taken out for all plots.

The principal density evolution compared to a single planet is about the same. However, with semi-major axis of $r_{1}=1$ and $r_{2}=1.32$, the planets have a different rotation period according to Kepler's law

$$
T_{1}^{2} / T_{2}^{2}=r_{1}^{3} / r_{2}^{3}
$$

Hence, the two planet create spiral waves which sweep over each other periodically (Fig. 10.1 and 10.2).

The potential vorticity $\zeta$ evolution shows according to Fig. 10.3 and 10.4 also inflection points as in the single planet cases. However, the $\zeta$ profiles here develops an instability first between the planets at $\Delta r \approx 5 r_{H}$ and not within the two PV peaks. The hypothesis here is that the two density "W" dips push the density between the two planets together such that $\zeta$ becomes deeper there and develops an instability sooner.

The total torque profile is affected by the vortex production at $\Delta r \approx 5 r_{H}$ and has therefore also a phase transition at $t \approx 100 P$. However, the torque evolution in Fig. 10.6 shows much higher oscillations also in phase I where the torque from a single planet was smooth. This is due to the massive spiral shock from the second planet sweeping by periodically. The torque from the second planet was not considered here. 


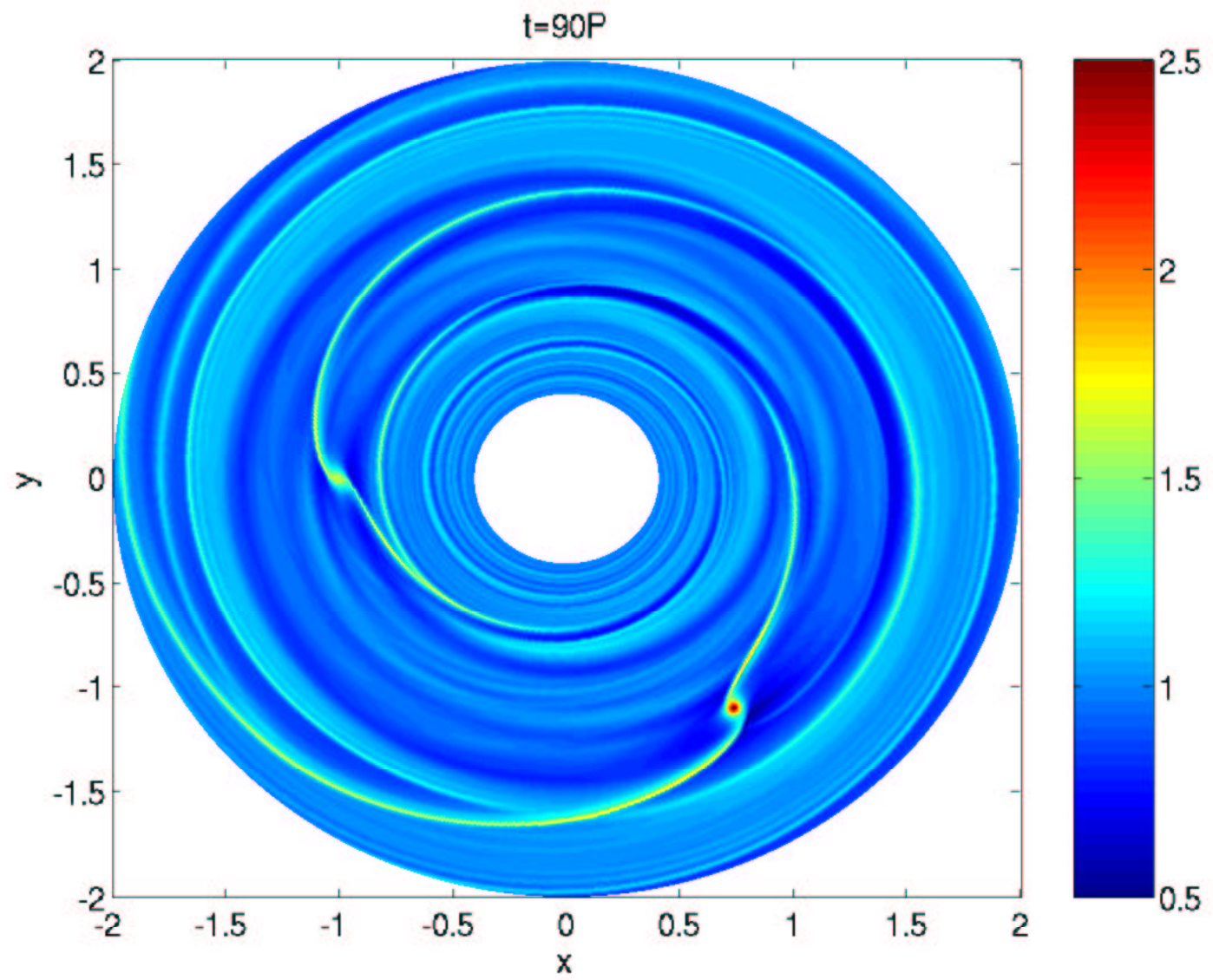

Figure 10.1: Density image with two planets at $r_{p 1}=1$ and $r_{p 2}=1.32$ both with a planet mass of $\mu=10^{-4}$. This plot is made at $t=90 P$ of the first planet. 


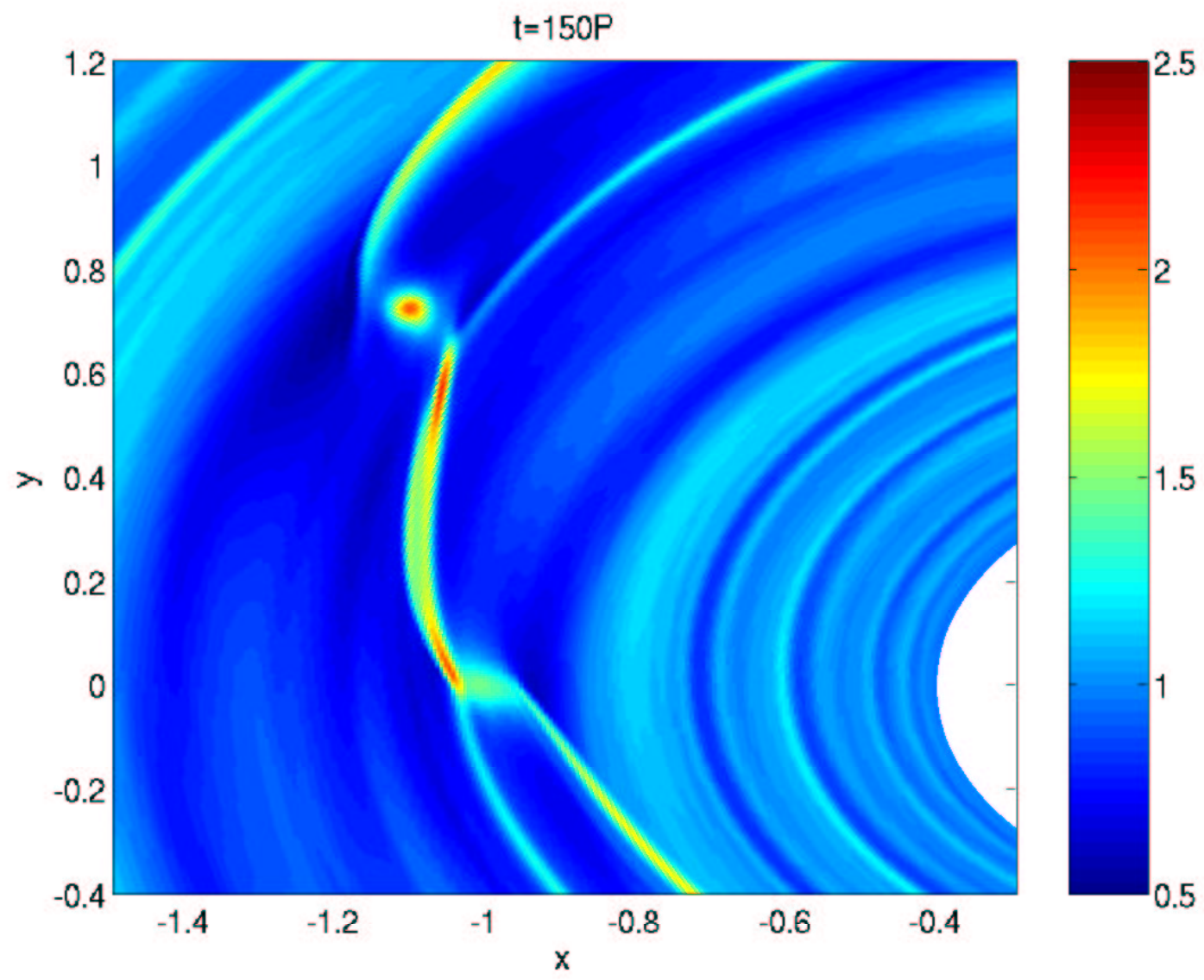

Figure 10.2: Zoom into the density image for two planets at $t=150 P$. Since the planets have a relative velocity to each other, also the spiral shocks cross each other multiple times. In this particular figure, the shock wave from the outer planet collides with the inner planet. 


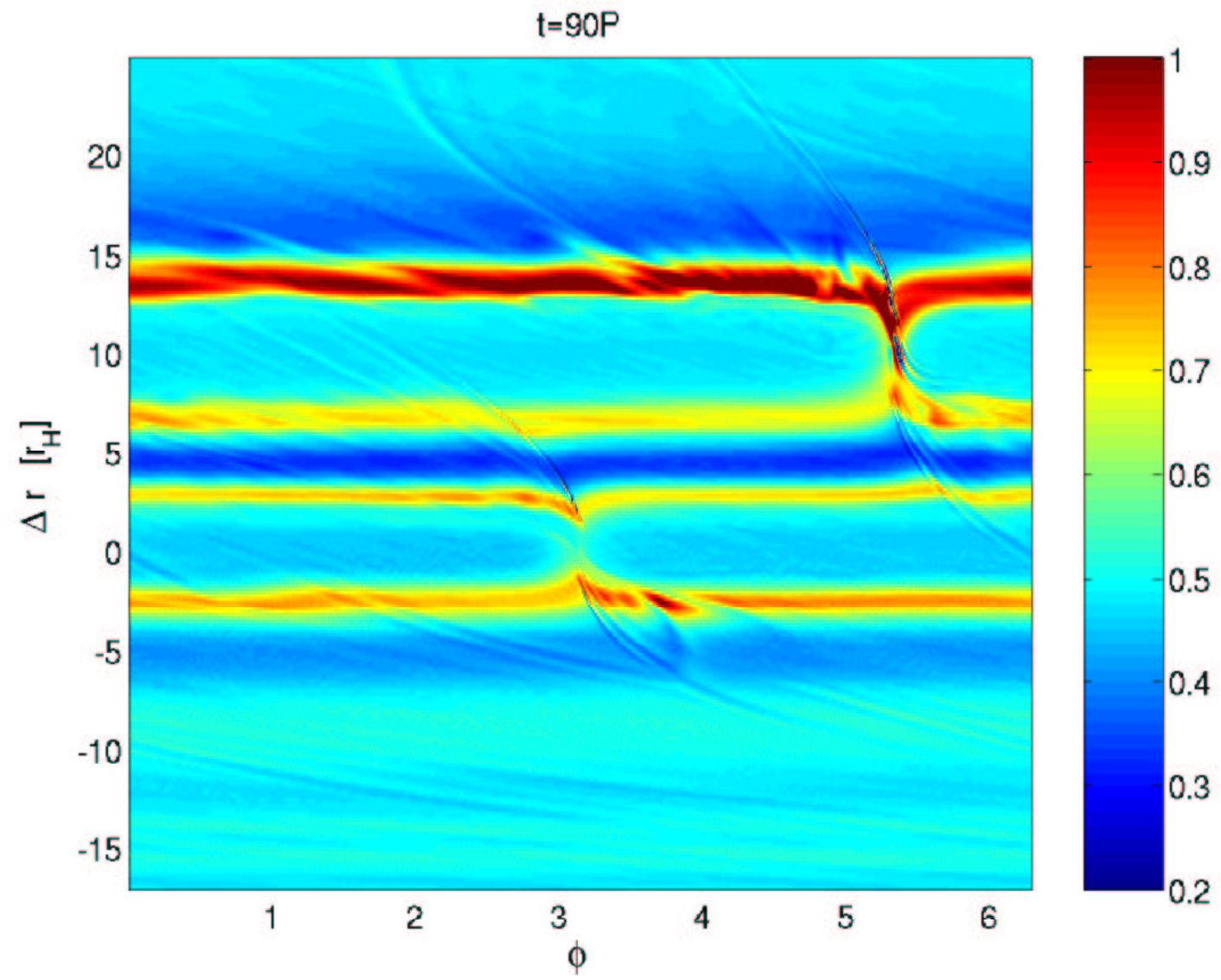

Figure 10.3: Potential vorticity of disk containing two planets at $t=90 P$. As with the single planet cases, the spiral shocks lead to inflection points in the potential vorticity. 


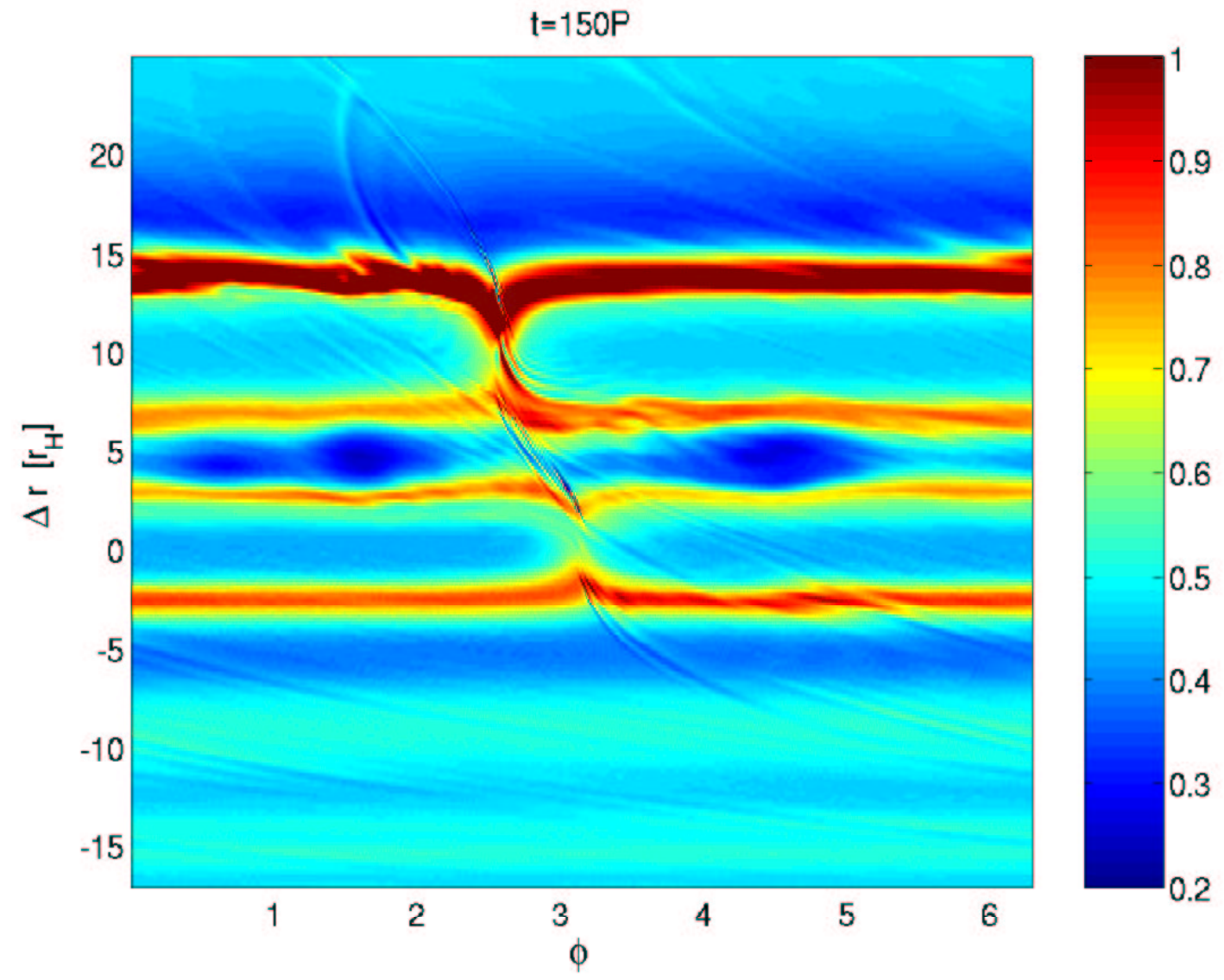

Figure 10.4: Same as Fig. 10.3 but at $t=150 P$ The potential vorticity valley at $\Delta r=5 r_{H}$ between the two planets becomes unstable and forms vortices. 


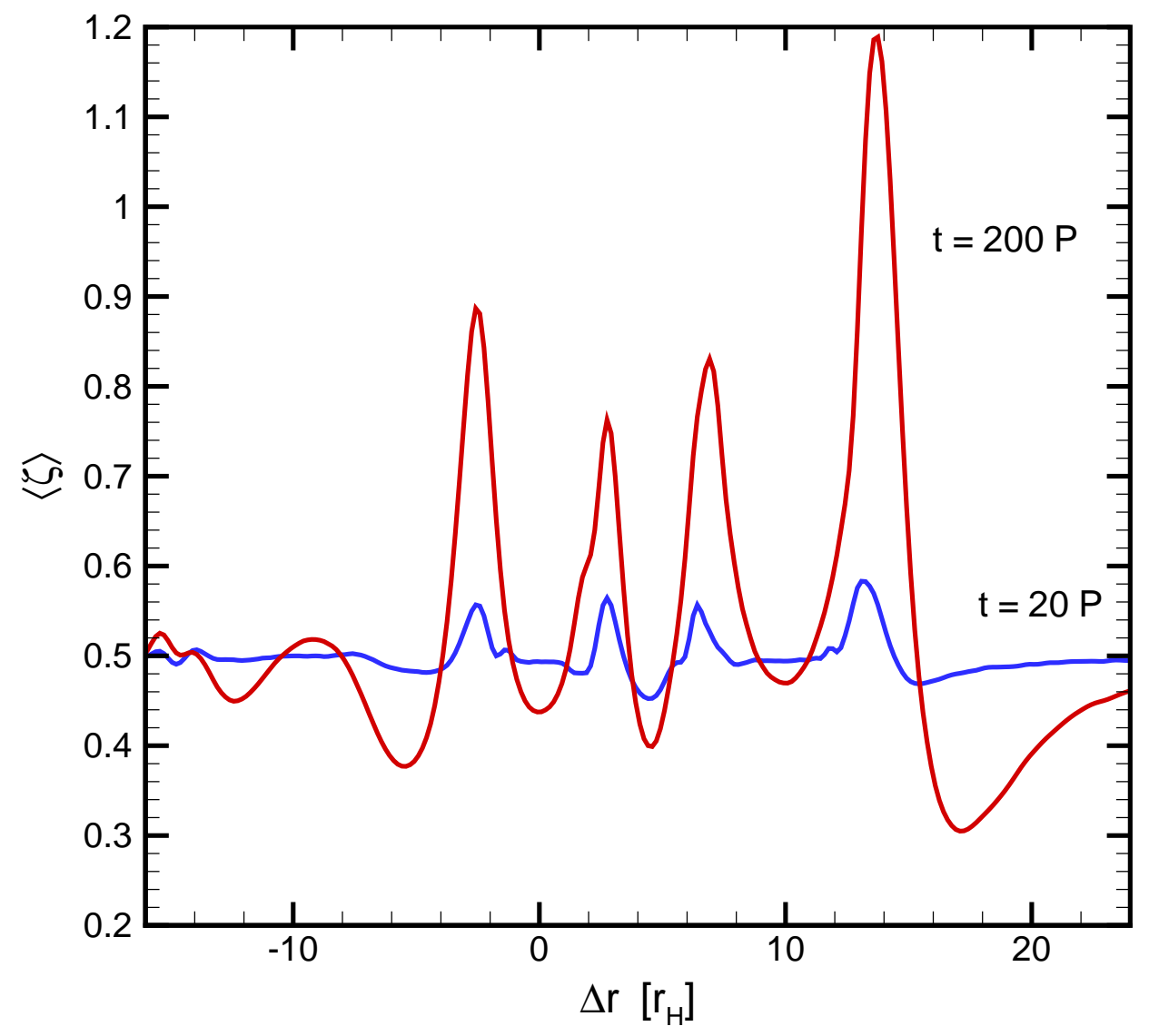

Figure 10.5: The PV profiles for two planets at $t=20 P$ (blue) and $t=200 P$ (red). This is similar to the single planet case where spiral shocks destroy the PV conservation and consequently inflection points form. This time, however, the region between the two planets at $\Delta r=5 r_{H}$ becomes unstable and develops vortices. 


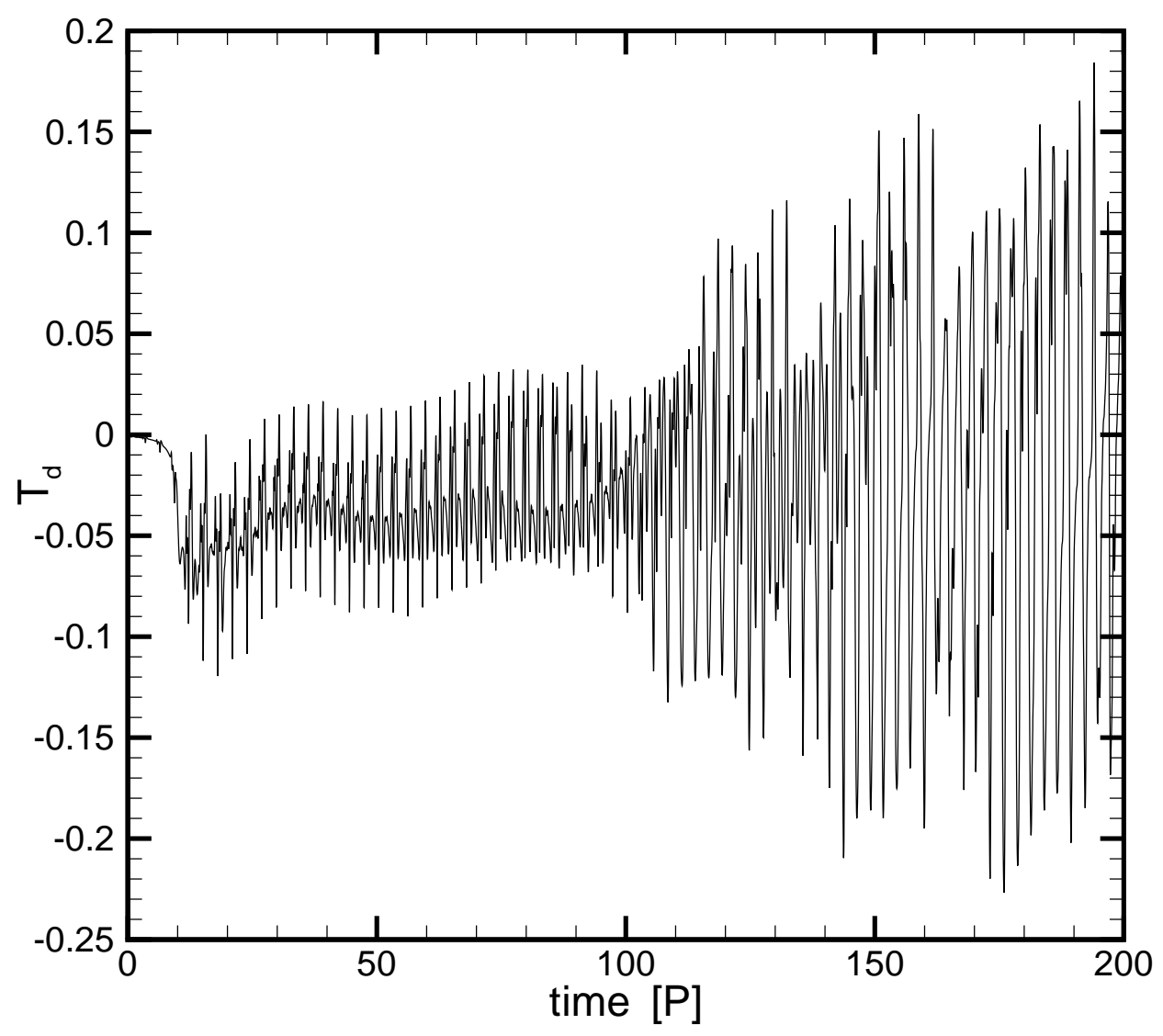

Figure 10.6: Torque evolution of the first planet in a multi planet case. Similar to Fig. 5.1 a phase transition takes place at $t \approx 100 P$. However, the torque is much noisier also in phase I due to the spiral shock of the second planet sweeping by frequently. 


\section{Chapter 11}

\section{Code Evaluation and Comparison}

\subsection{Steady State Disk Test}

One of the most important tests in accretion disk simulations is the Steady State Disk Test. In theory, if a thin disk does not suffer any perturbation or outside forces, it should not collapse, i.e., the radial velocity $v_{r}$ should stay at zero. A disk can be setup such that the gravitational force, the centrifugal force, and a pressure gradient force cancel each other. The initial conditions for a stable steady-state disk are therefore

$$
\begin{aligned}
\Sigma(r) & =r^{-\beta} \\
v_{r} & =0 \\
v_{\phi} & =\sqrt{1+c_{s}^{2} r^{2} \frac{\Sigma^{\prime}(r)}{\Sigma(r)}} \sqrt{\frac{1}{r}}
\end{aligned}
$$


where $\Sigma^{\prime}(r)=d \Sigma(r) / d r$. The equations for $v_{\phi}$ is only valid for an isothermal equation of state.

Since every numerical scheme, especially the finite differencing scheme, is not able to capture gradients very well, an easy test is to set up a steady state disk and check how the radial velocity $v_{r}$ is evolving. It should stay close to zero.

Fig. 11.1 shows that indeed the numerical hybrid (see $\S 2)$ scheme handles the steady state disk well. The maximum radial velocity is to the order of $10^{-5}$. That means, that also the time scale is to the order of $10^{5}$ years. That is way longer than other effects in the disk and, hence, can be neglected.

\subsection{Resolution Tests}

Another important test for finite grid schemes is the resolution test which compares the results at certain times from different grid cell sizes. First of all, a two-dimensional grid size was chosen such that it would be almost square at the planets radius. That is why the number of azimuthal grid points is almost a factor of four in the disk dimensions with $r_{\min }=0.4$ and $r_{\max }=2$. A number of different grid sizes was selected and the end results are compared. Fig. 11.2 compares the density close to the planet from three different grid sizes, i.e. $N_{r} \times N_{\phi}=200 \mathrm{x} 800$, $300 \times 1200$, and $400 \times 1600$. As we see in this figure, the density profile is almost identical.

Since we are mostly concerned about potential vorticity, Fig. 11.3 shows $\zeta$ 


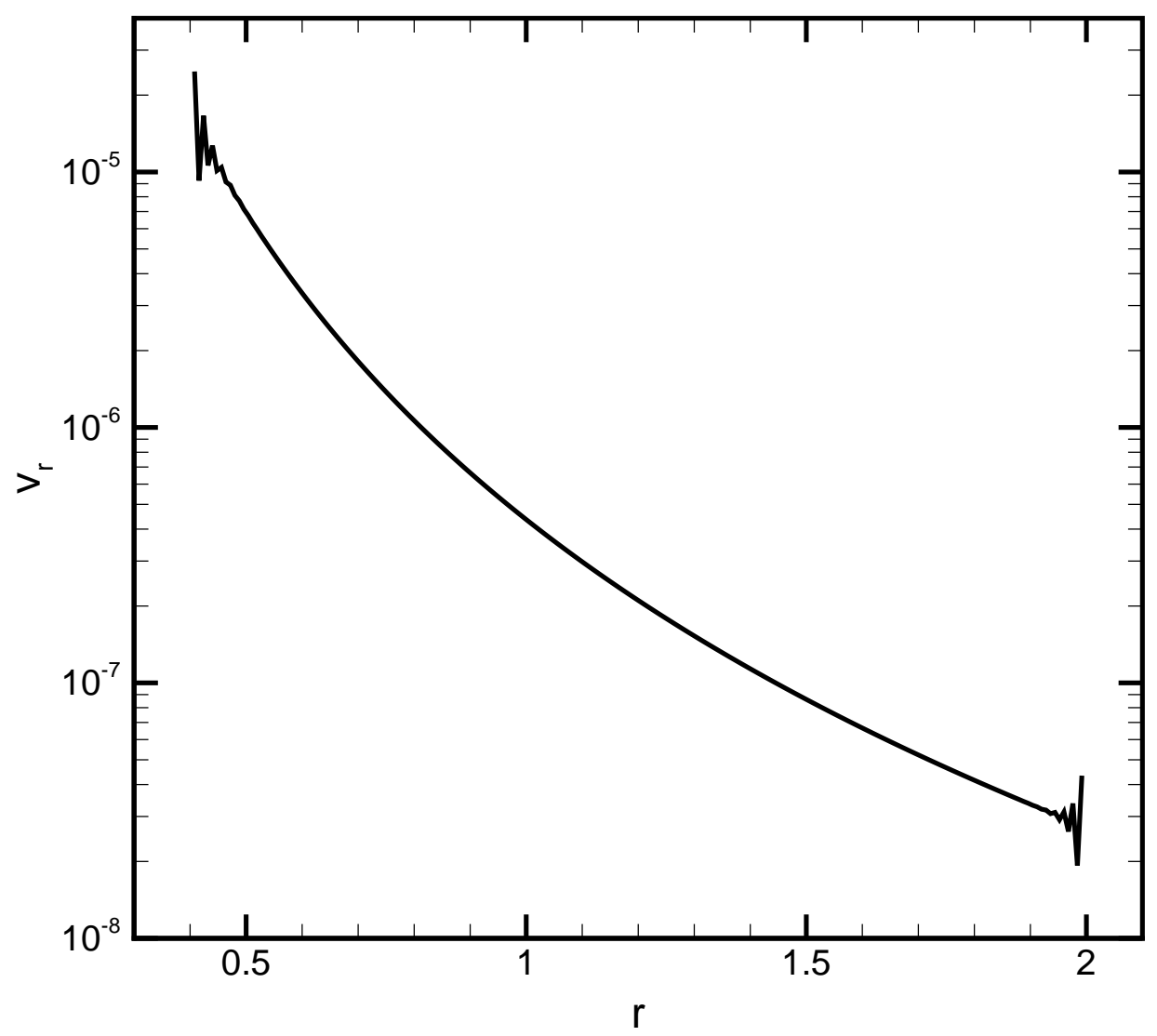

Figure 11.1: Radial velocity for a steady state disk. After $t=10 P$ the disk reaches a steady state with a small radial velocity. This plot here is made at $t=100 P$. The highest values are at the inner boundary with $v_{r} \approx 10^{-5}$ and remain at that level as long as the code was tested (up to $1000 P$ ). At $r=1$, where we put the planet for real simulations, the radial velocity is one magnitude smaller and becomes even smaller at the outer boundary. 
profiles from the same resolution runs as before. Potential vorticity is a second order effect because it involves radial and azimuthal derivatives. Hence, the picture looks somewhat different by comparing potential vorticity. The inflection points are still at the same radial distance. However, their magnitude differs from resolution to resolution. The growth rate in region III (see Fig. 9.8) does not depend much on resolution. Still, regions II and I are affected by resolution and their growth rate differs by $60 \%$. Also, there is a slight shift in radius for the minima at $\approx \pm 2 r_{H}$ (region I). However, the slope steepens with increasing resolution and therefore, secondary instabilities still unfold at about the same time even though the minima are not as deep. This is still a somewhat disturbing trend because the question remains if the dip in region I will eventually vanish for very high resolution. As it was discussed in $\S 9$, the predictions from the analytical expression Eq. (9.3) match the potential vorticity evolution well in region III and II but region I still remains uncertain to this point. Unfortunately, region I becomes unstable first developing vortices which have a large effect on the disk and the torque evolution.

The rest of the disk is, to a large degree, independent of resolution. The central disk around the planet depends somewhat on the chosen grid size since the sampling of the gravitational potential will also depend on the resolution. 


\subsection{Comparison with VH1}

Another way to test a code is to compare it with a different numerical scheme. VH1 (Virginia Hydrodynamic 1), written by the numerical astrophysics group at the University of Virginia, was chosen for this purpose. The numerical scheme is based on Colella \& Woodward (1984) applying a piece-wise parabolic method (PPM). VH1 is written as a Lagrangian hydrodynamics code coupled with a remap onto the original Eulerian grid. The package including a manual can be downloaded from http://wonka.physics.ncsu.edu/pub/VH-1/.

All codes, VH1 as the hybrid code, have strengths and limitations. In the case of a protoplanet embedded in a disk, it is difficult to ascertain which code gives the correct result. Both version show an overall agreement in term of density evolution. However, when it comes to second order effects like potential vorticity, it is unclear which is closer to reality. There is no general test problem so far that can be solved analytically like the Sod Test problem (see Toro, 1999).

VH1 was converted to handle an accretion disk with a planet and an isothermal equation of state. Again the density evolution is at the same order of magnitude and shows the same trends. The potential vorticity image (Fig. 11.4) is quite noisy in VH1 but the overall trends of inflection points are there. An azimuthally averaged PV profile in Fig. 11.5 tells us that the growth rate in VH1 is about twice as large as in the hybrid code. We also see that the inflection points are at the same radial location. 
Since the hybrid code is highly optimized to the disk problem, including the locally co-moving frames and partial time stepping, it is a factor of four faster than VH1. Moreover, the hybrid code is parallelized with Open/MP and gains almost another factor of two in speed when used on a shared memory machine. 


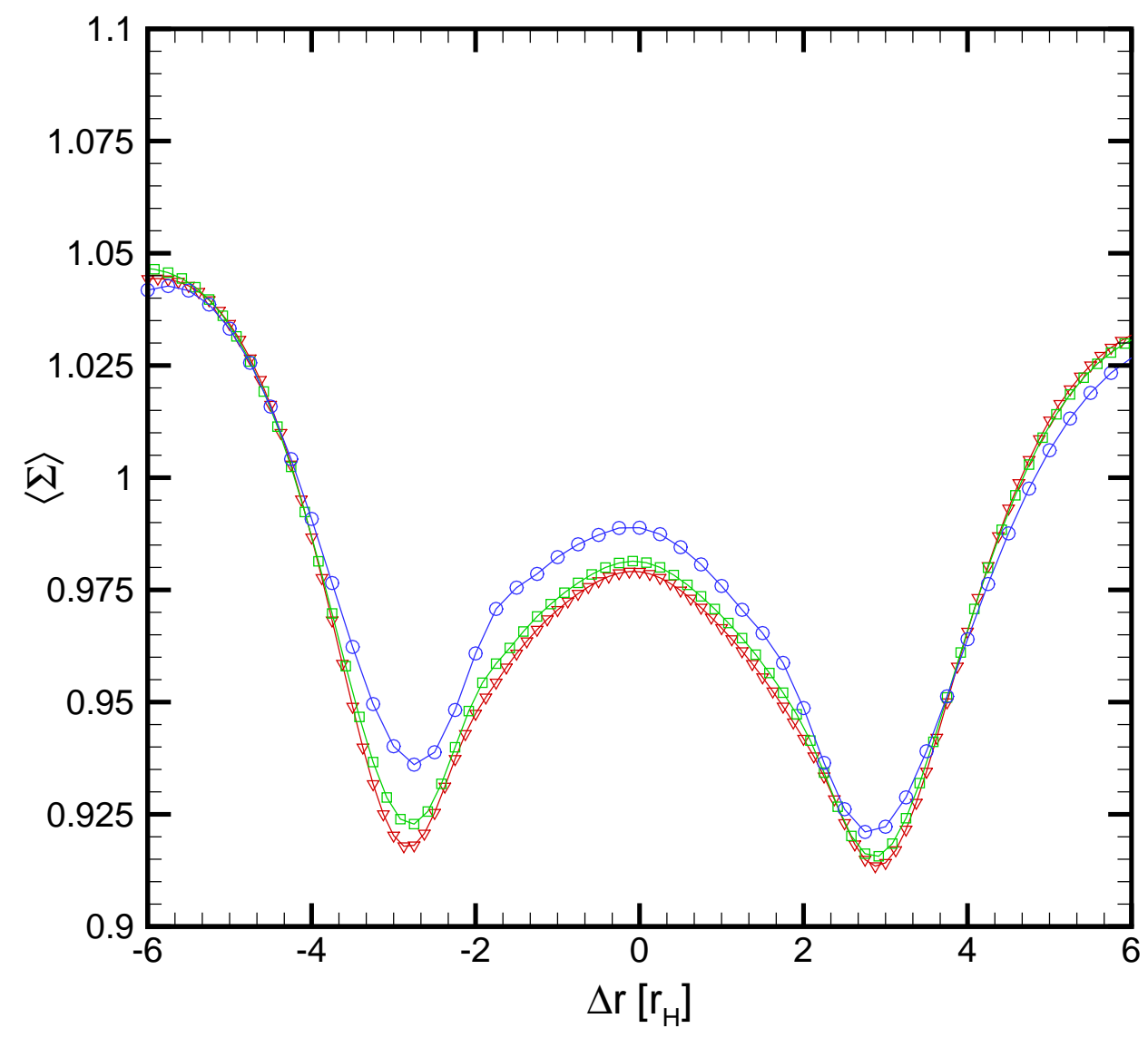

Figure 11.2: Comparison of azimuthally averaged density with different grid resolutions at $t=30 P$. The applied grid resolutions are 200x800 (blue circles), 300x1200 (green green), and 400x1600 (red triangles). Here, only the region around the planet is shown. The curves, especially for the two higher resolutions agree very well. 


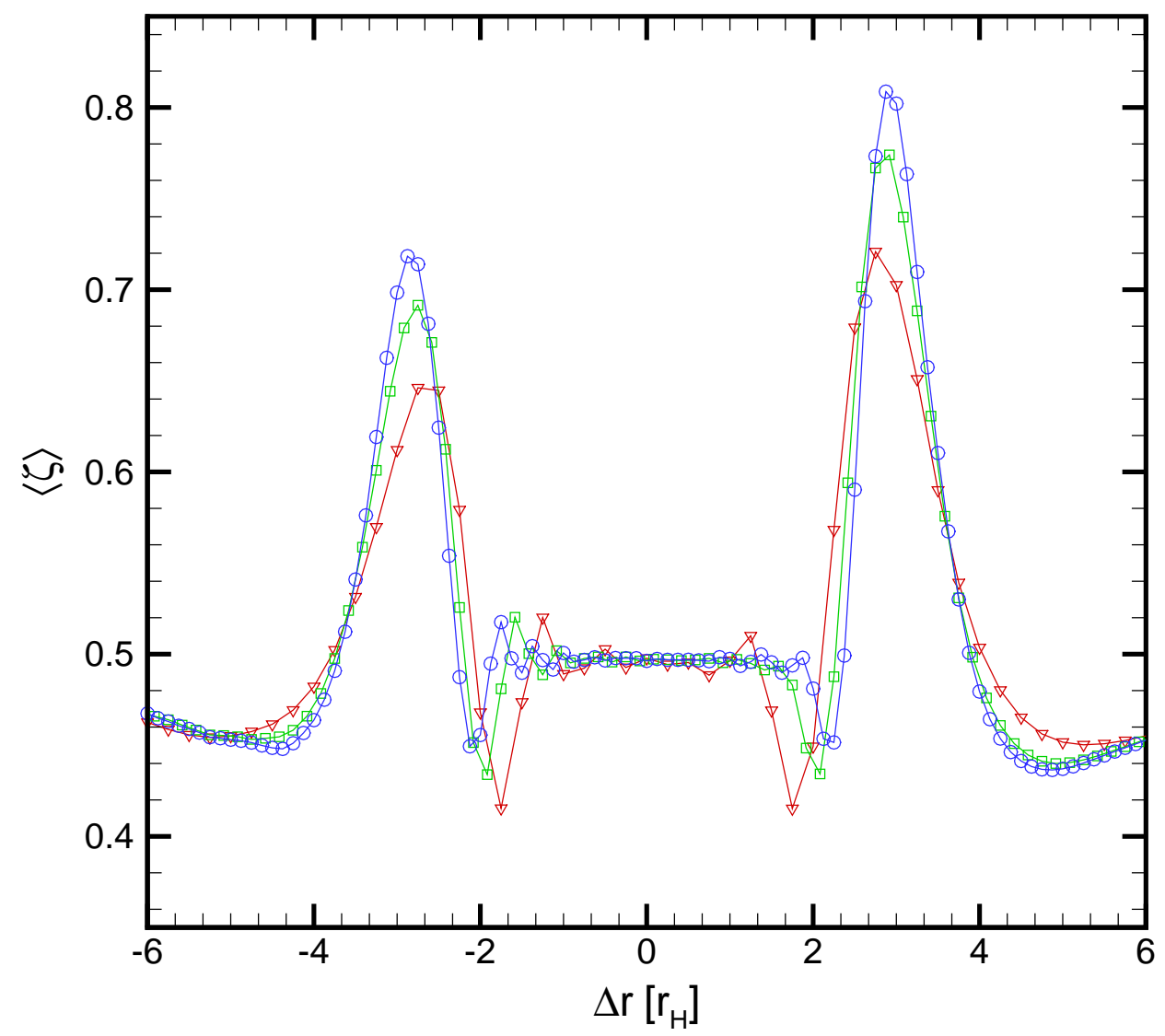

Figure 11.3: Azimuthally averaged potential vorticity with the same parameters as in Fig. 11.2. The applied grid resolutions are 200x800 (red triangles), 300x1200 (green boxes), and $400 \times 1600$ (blue circles) at $t=30 P$. Even though the PV is a second order effect due to differential nature, the agreement is still quite good. The growth rate seems to differ slightly. However, the peaks are at the same location, and the central values are also quite well approximated with the lower resolution runs. 

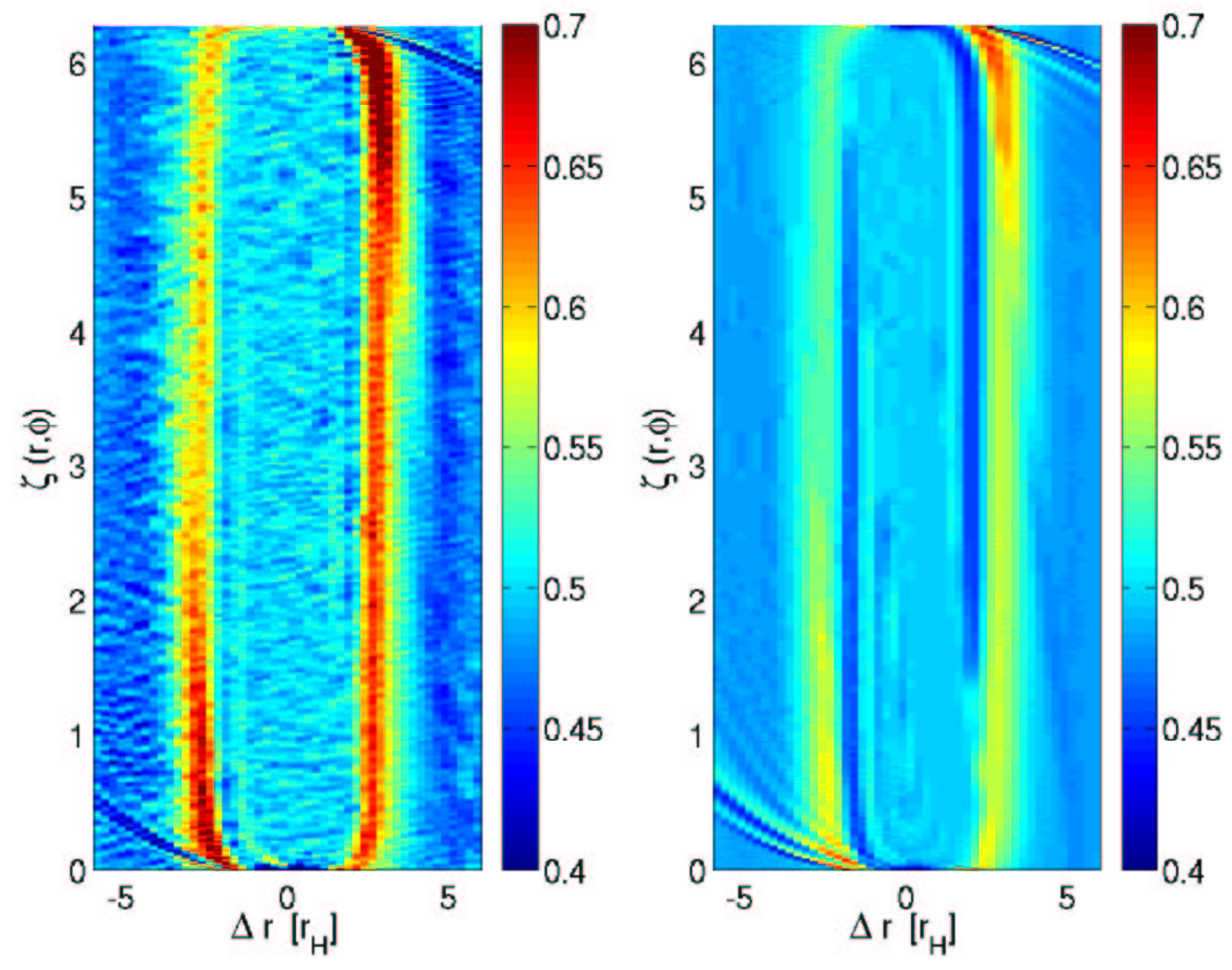

Figure 11.4: Comparison of potential vorticity images from two different codes, VH1 (left) and the hybrid code (right). The growth rate in VH1 is about a factor of two larger but the overall disk seems much noisier. The general features (peaks and minima) are visible at the expected locations. This plot was made at $t=10 P$ with a resolution of $200 \times 800$. 


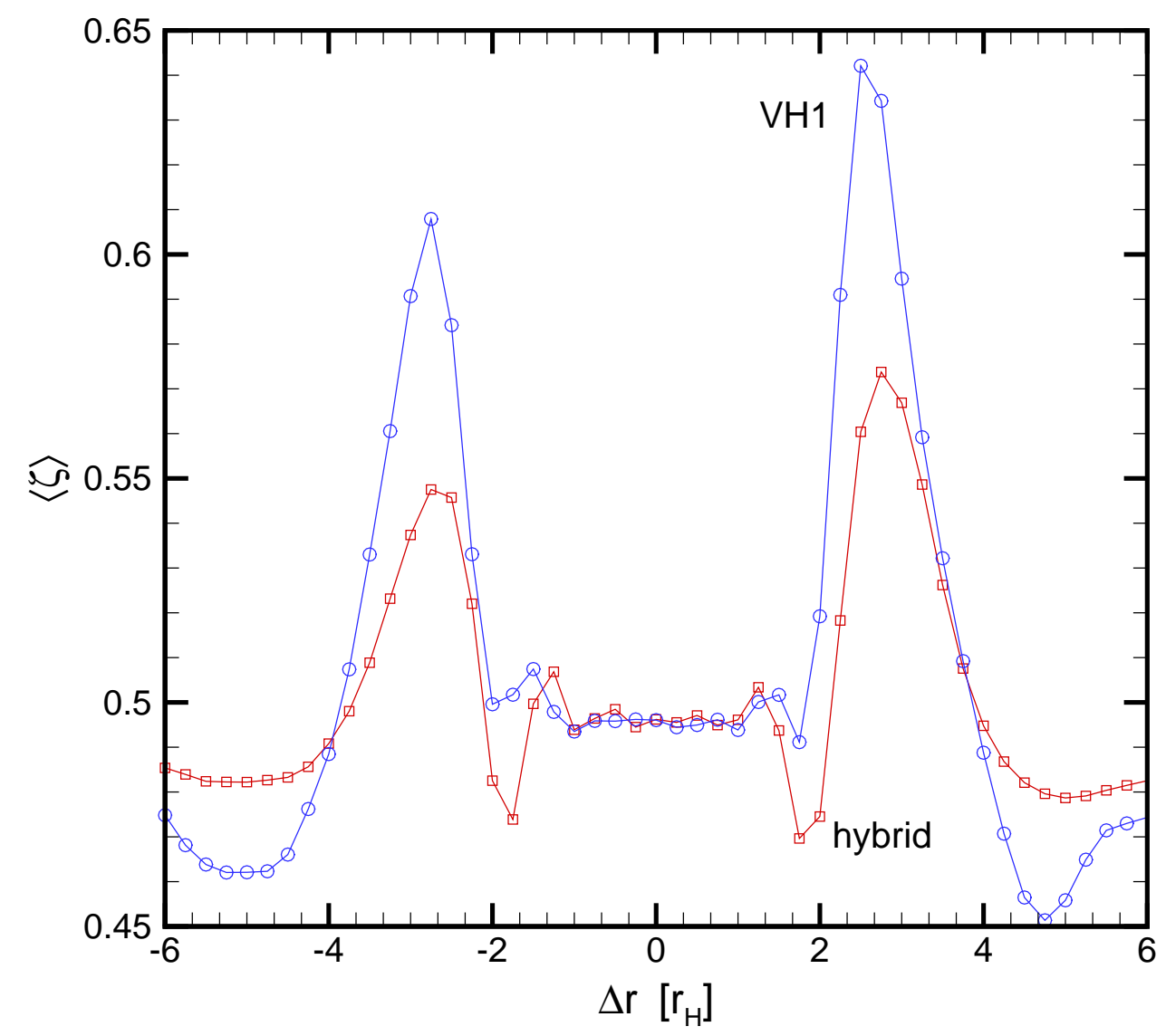

Figure 11.5: Azimuthally averaged PV with the same parameters as in Fig. 11.4. The growth rate with VH1 (blue circles) seems to be a factor of two larger that the growth rate with the hybrid code (red boxes) but the location of inflection points is as expected. 


\section{Chapter 12}

\section{Restricted Three-Body Approach}

\subsection{Initial setup and orbital properties}

Particle paths are investigated with a restricted three-body code which solves the equation of motion in the synodic system, i.e. the direction of the x-axis is chosen such that the two masses always lie along it. The coordinates of the primary are $\left(x_{1}, y_{1}\right)=\left(-\mu_{2}, 0\right)$ with a relative mass of $\mu_{1}=1-\mu_{2}$. The secondary is located at $\left(x_{2}, y_{2}\right)=\left(\mu_{1}, 0\right)$ with a relative mass of $\mu_{2}=m_{2} /\left(m_{1}+m_{2}\right)$. The two bodies have a constant separation and the same angular velocity $\omega$ around their common center of mass. For the current simulations $\mu_{2} / \mu_{1}=1 \times 10^{-4}$ is chosen.

The distance from the particle to the star is $r_{1}=\left[\left(x+\mu_{2}\right)^{2}+y^{2}\right]^{1 / 2}$ and the distance to the planet $r_{2}=\left[\left(x-\mu_{1}\right)^{2}+y^{2}\right]^{1 / 2}$ where $(x, y)$ are the coordinates of the particle with respect to the rotating or synodic system. 
The equation of motion in this system becomes

$$
\begin{gathered}
\ddot{x}-2 \omega \dot{y}-\omega^{2} x=-\left[\mu_{1} \frac{x+\mu_{2}}{r_{1}^{3}}+\mu_{2} \frac{x-\mu_{1}}{r_{2}^{3}}\right] \\
\ddot{y}+2 \omega \dot{x}-\omega^{2} y=-y\left[\frac{\mu_{1}}{r_{1}^{3}}+\frac{\mu_{2}}{r_{2}^{3}}\right]
\end{gathered}
$$

where $\omega=1$ is the angular velocity of the rotating frame.

The equation of motion are solved with a Fourth Order Runge-Kutta scheme using adaptive time steps. By closely monitoring the Jacoby constant, the quality of the integration of motion is assured. The Jacoby constant is

$$
C_{j}=\omega^{2}\left(x^{2}+y^{2}\right)+\left(\frac{\mu_{1}}{r_{1}}+\frac{\mu_{2}}{r_{2}}\right)-\dot{x}^{2}-\dot{y}^{2}
$$

which is the only constant of motion in this system.

The simulations showed that the mean change of $\left(\overline{\Delta C_{j}}\right) \approx 2.4 \times 10^{-12}$. Only a few particles are lost from the system, especially if they are initially located in the region $r_{H}<|\Delta r|<\sqrt{12} r_{H}$, the so-called separatrix region.

In this system where two masses $\mu_{1}$ and $\mu_{2}$ move on circular orbits around their common center of mass, their positions are stationary in a frame rotating with an angular velocity equal to the mean motion $\omega$ of either mass. Although the circular restricted three-body problem is not integrable one can find a number of special 
solutions (libration points) where particles have zero velocity and no acceleration in the rotating frame (see Murray \& Dermott, 1999). The libration points $L_{4}$ and $L_{5}$ are defined as the leading and trailing equilibrium points respectively. The location of $L_{3}$ is on the opposite of the primary on a straight line between primary and secondary (Fig. 12.1). Particles with a small displacement from $L_{4}$ or $L_{5}$ have typical libration eigenvalues of

$$
\begin{array}{r}
i \lambda_{1,2} \approx \pm \sqrt{1-\frac{27}{4} \mu_{2}} \\
i \lambda_{3,4} \approx \pm \sqrt{\frac{27}{4} \mu_{2}}
\end{array}
$$

With these eigenvalues the periods are then $T_{L}=2 \pi /|\lambda|$. In our case where $\mu_{2}=10^{-4}$ one finds $T_{L}=38.5 P$ with $P$ as the period of the rotating frame, i.e., the period for the secondary orbiting the primary. Note, this expression is only valid for small numbers of $\mu_{2}$ and small displacement from $L_{4}$ or $L_{5}$.

Several runs with test particles in this system were made. The initial velocity of a particle is set to the Keplerian velocity in the corotating coordinate system $v_{\phi}=\sqrt{\left(1 / r_{1}\right)}-r \omega$. By locating particles in the vicinity of $L_{3}$ three different regions are found: Region 1: Particles with $|\Delta r|=\left|r-r_{L_{3}}\right|<r_{H}$ carry out horseshoe orbits encircling $L_{3}, L_{4}$ and $L_{5}$. The closest approach to the secondary is still far enough so that the particle is not much perturbed and only deflected by the planet. All orbits in this region are closed. Periods for particles in the 

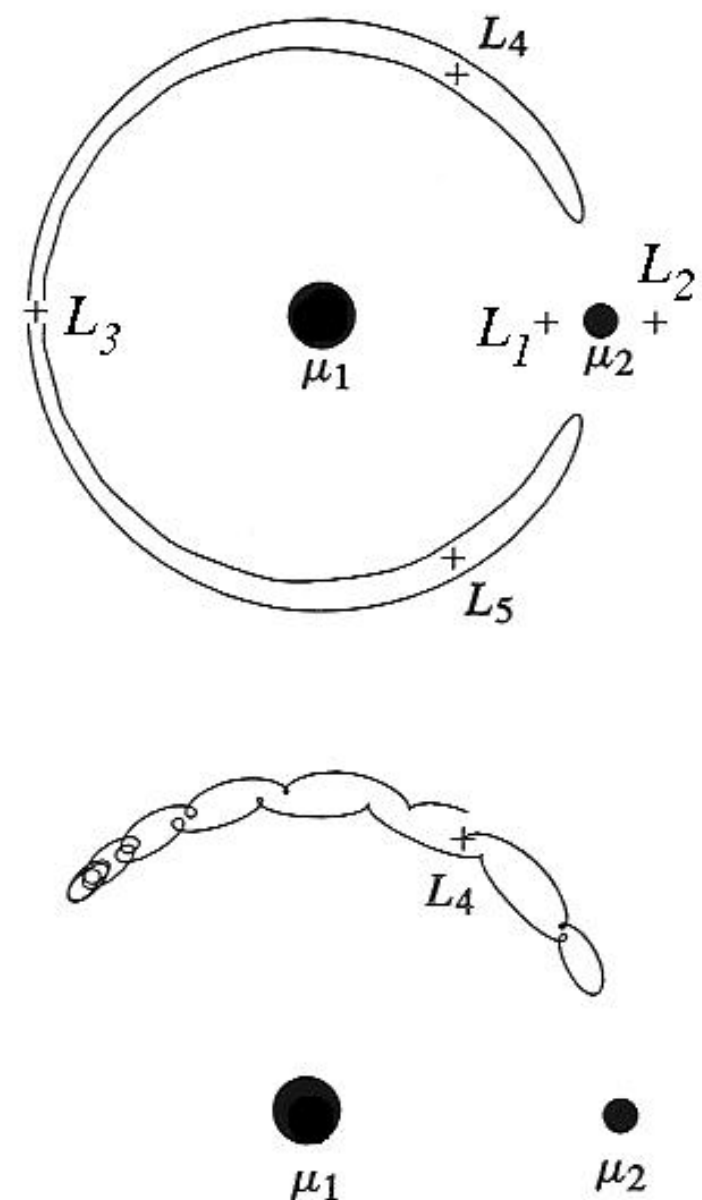

Figure 12.1: Two typical orbits in the three-body problem. The (upper) horseshoe orbit encloses three libration points $L_{3}, L_{4}$, and $L_{5}$. The (lower) tadpole orbit encloses only one libration point $L_{4}$ or $L_{5}$. In the three-body problem, the motion can be divided into epicyclic motion and the motion of the epicenter. In the second example the tadpole orbit shows both motions whereas the horseshoe orbit has almost no epicyclic motion and largely an epicenter motion only. Figure taken from Murray \& Dermott (1999). 
horseshoe orbit are plotted in Fig. 12.2. Test particles from this plot were initially located at a constant $\phi$ for a range of $r$ around $L_{3}$. Dermott \& Murray (1981) provide a time-scale to loose particles from this orbit with $\Gamma<P / \mu_{2}^{5 / 3}$ where $P$ is the orbital period of the secondary.

Region 1 contains also a subgroup of orbits, the so-called tadpole orbits. Fig. 12.1 shows a horseshoe and a tadpole orbit. Dermott \& Murray (1981) discussed that for regions with $C_{j}<3+5 \mu_{2}$ tadpole orbits are possible. These particles are located around $L_{4}$ and $L_{5}$ because these are the only regions where the condition above is satisfied. This is confirmed by the restricted three body simulations here. The period has a lower limit of $P>1 / \sqrt{27 \mu_{2} / 4} \approx 38$ in units of orbital periods of the secondary. By increasing $\Delta r$ the period is slightly increasing, too, until the condition $C_{j}<3+5 \mu_{2}$ or $\Delta r<r_{R} / 2$ is not satisfied and the orbit switches from a tadpole orbit to a horseshoe orbit. The period jumps to a large value and decreases with further $\Delta r$ (see Fig. 12.3).

Particles with initial conditions in Region $2\left(r_{H}<|\Delta r|<\sqrt{12} r_{H}\right)$ come very close to the secondary and experience a large perturbation driving the orbital eccentricity of the particle. They are scattered by the planet and as a result particles cross $r=1$ line and bounce in the synodic system reaching large $|\Delta r(t)|$ (see Fig. 12.4). However, at all times it is confined by an $r_{\max }$ and $r_{\min }$ which can be calculated from $C_{j}$ and zero velocity curves (Murray \& Dermott, 1999). Particles starting within this region are not necessarily lost but they have a much 


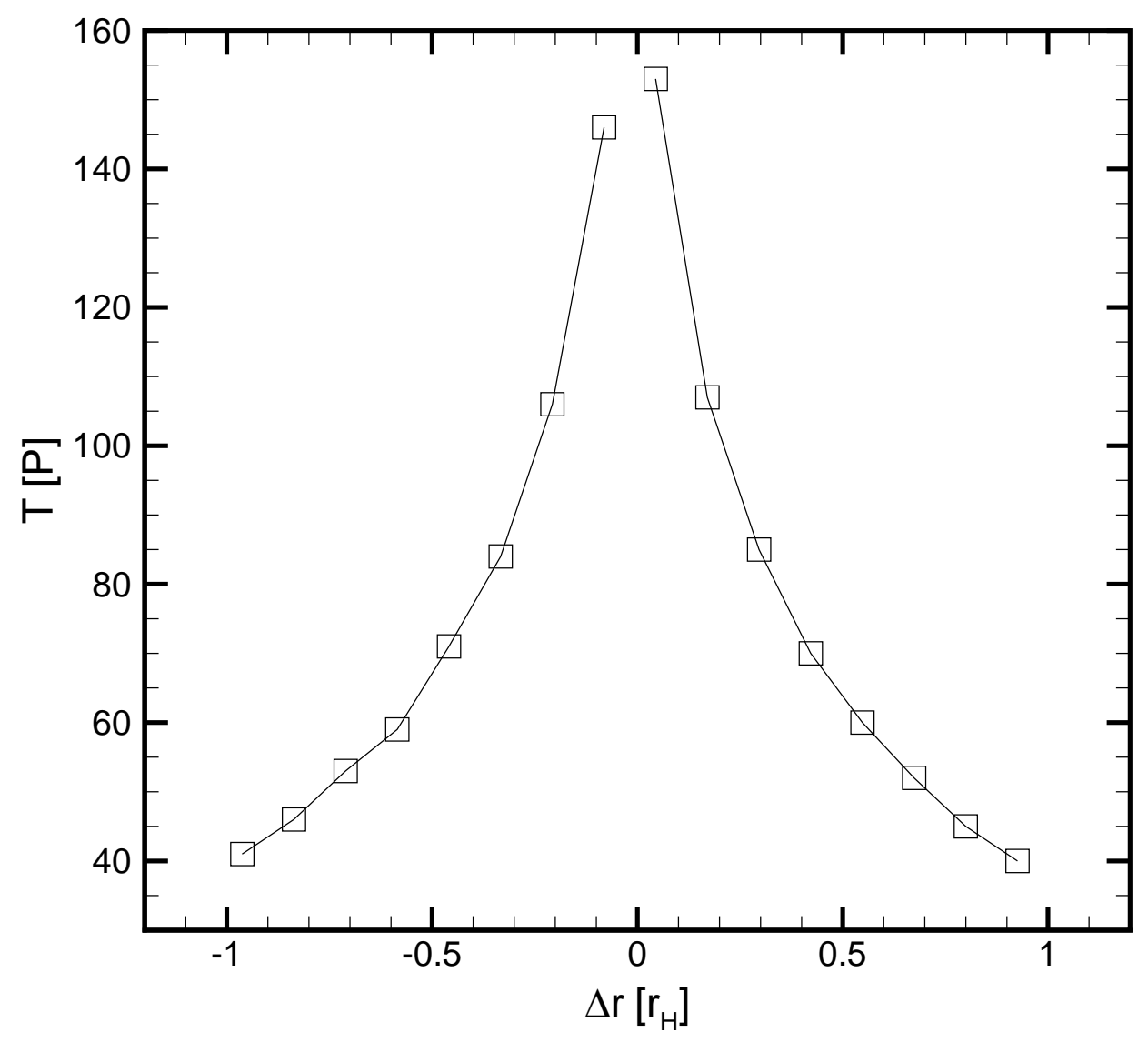

Figure 12.2: Libration periods for particles around $L_{3}$. The abscissa is the distance to $L_{3}$ is in units of $r_{H}$. Particles with increasing distance to the libration point have a decreasing period. A particle located exactly at $L_{3}$ would have an infinite period and would not move at all. However, this point is unstable and the slightest perturbation would move it into a horseshoe orbit. 


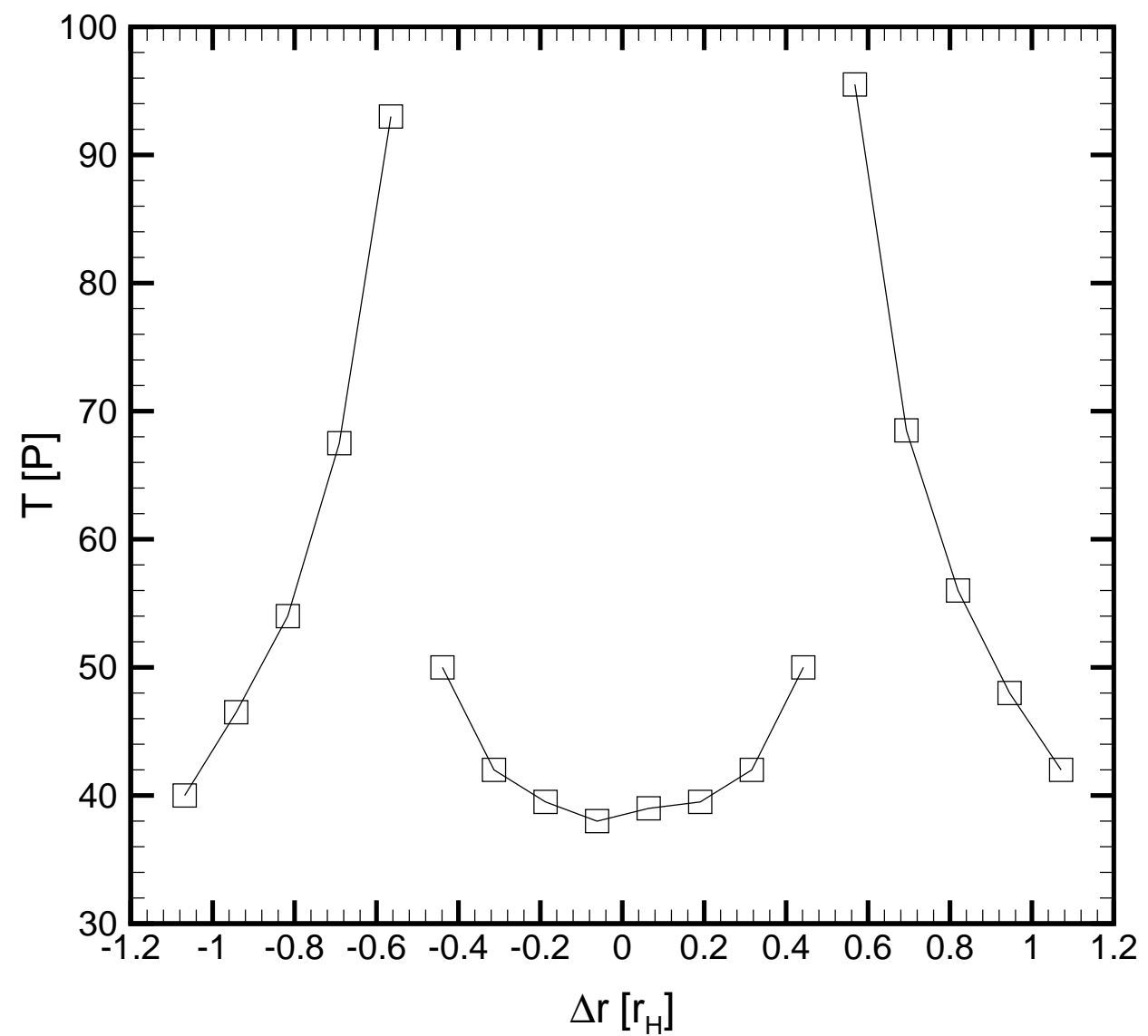

Figure 12.3: Libration periods of particles around $L_{4}$. Placing a number of particles radially around $L_{4}$ enables to measure their libration periods. A minimum period of $T \approx 38 P$ is found for particles very close to $L_{4}$. Once the initial condition exceeds $|\Delta r|>0.5 r_{H}$, the particle orbit switches from a tadpole orbit to a horseshoe orbit with a much larger period. 
larger area for allowed orbits. They are distributed between $r_{\min }$ and $r_{\max }$. But $\left|1-r_{\max }\right|>\sqrt{12} r_{H}$ and therefore the number density drops within $r_{H}<|\Delta r|<$ $\sqrt{12} r_{H} \cdot$

Particles in Region 3 are on streaming orbits. Even the closest approach to the planet is not close enough so that these orbits are not significantly disturbed. The particle orbits the primary in an almost circular shape with small eccentricities. The orbits are closed and stable.

For measuring the periods of these orbits a series of test particles are placed over a large radial range around $L_{4}$ (Fig. 12.4). The idea is to figure out what is causing the oscillation in torque phase I (Fig. 7.2). The minimum period of $T \approx 38 P$ for particles very close to $L_{4}$ is in agreement with the analytical expectation of Eq. (12.5). Moving further away from $L_{4}$, a discontinuity in the periods appears at $0.5 r_{H}$. At that point the orbit switches from a tadpole orbit to a horseshoe orbit with a much longer period $T \approx 92 P$. Then the period decreases again for particles further away from $L_{4}$, reaching a minimum of about $40 P$ at $|\Delta r| \lesssim r_{H}$

The oscillation period in the torque evolution in phase I (Fig. 7.2) is due to particles in tadpole and horseshoe orbits. These orbits have the closest approach to the planet and therefore the torque should be the strongest from the material in these orbits. Since the periods are distributed over quite a large range between $38 P$ and $92 P$, only the ones with the closest approach will have the largest contribution. 


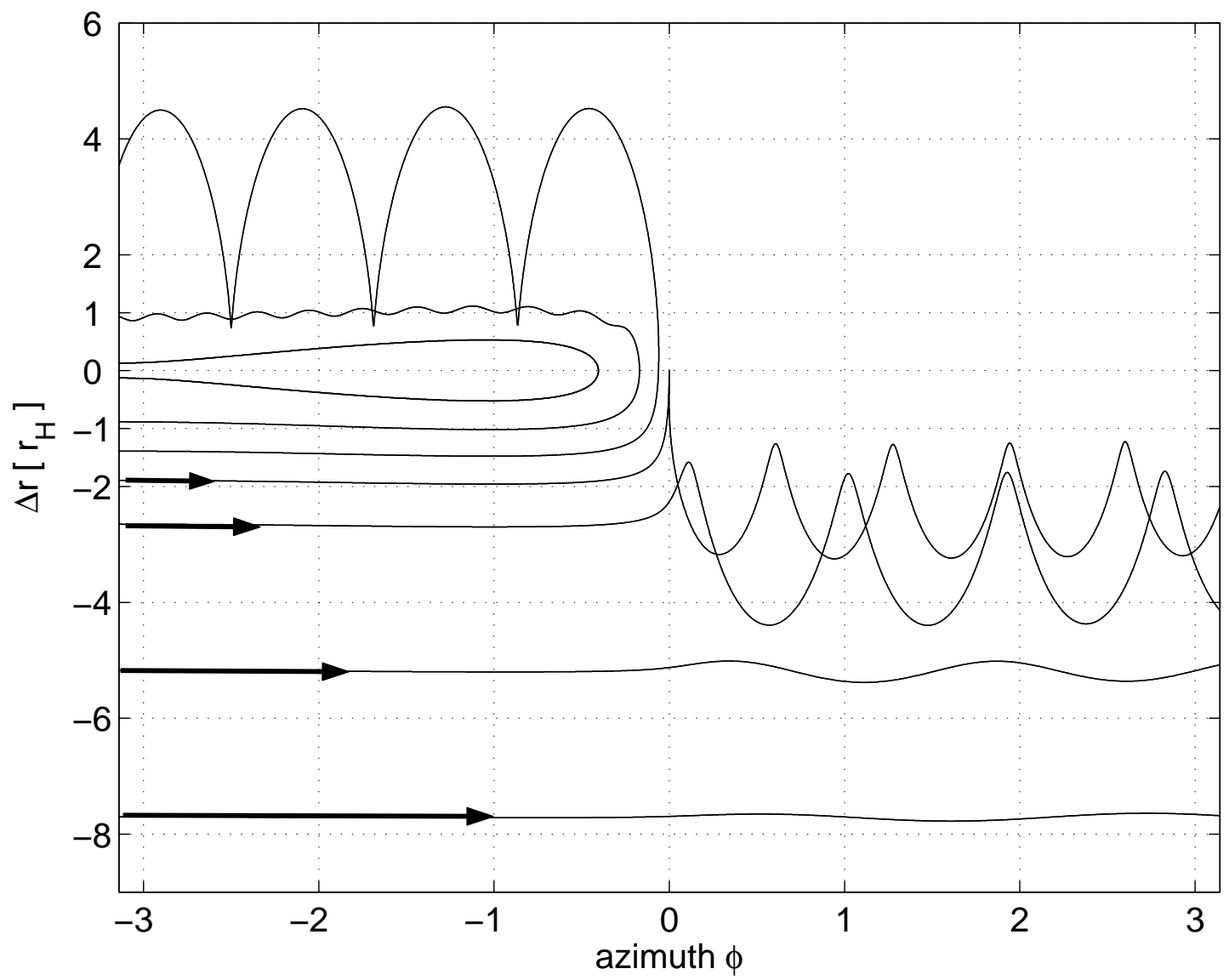

Figure 12.4: Particle trajectories obtained by solving the restricted three-body equations. The perturbing planet is located at $(\Delta r, \phi)=(0,0)$. The particles start in circular orbits from the left. There are three types or regions for particles paths in this system: Region 1 particles with initial $|\Delta r|<r_{H}$ execute horseshoe orbits and are only marginally perturbed by the planet. Region 2 particles with an initial $r_{H}<|\Delta r|<\sqrt{12} r_{H}$ (separatrix) suffer close encounters with the planet and are highly perturbed. Region 3 particle $\left(|\Delta r|>\sqrt{12} r_{H}\right)$ are on streaming orbits and experience only a small deviation from the initial circular orbit. See also Murray \& Dermott (1999). 
Orbits with the closest approach to the planet are at $|\Delta r| \lesssim r_{H}$, i.e. just inside the horseshoe region. Particles just outside the horseshoe region will get even closer to the planet but they are kicked into high eccentricity orbits. Their orbits are chaotic. A period for these particles cannot be defined because they are not closed and because they switch frequently between horseshoe and streaming like orbits.

\subsection{Results from the Three-Body Simulations}

Petit \& Henon (1988) studied gap formation in planetary rings using a MonteCarlo approach with 1000 particles. The effect of gap formation was predicted as a consequence of the deflective effect of the secondary (Lissauer et al., 1981; Henon, 1981).

Using this restricted three-body approach with one million particles randomly placed between $|\Delta r|<6 r_{H}$ and $0<\phi<2 \pi$, the results in Petit \& Henon (1988) are confirmed. After $t=300 P$ many particles from the region between the streaming and the horseshoe region (i.e., Region 2: $r_{H}<|\Delta r|<\sqrt{12} r_{H}$ ) are strongly perturbed by close encounters with the planet. Their number density dramatically drops in this regions because their orbits are distributed over a larger area. It is shown in Fig. 12.5 the density drops in two channels at $\approx 2 r_{H}$ matching the density channels from our hydro-code simulations with the same planet mass $\mu=10^{-4}$. Since the hydro-code and three-body simulations agree so well in the azimuthally averaged density (Fig. 12.6), this implies that the pressure forces don't play a 
crucial role. For low sound speed simulations the three-body code can be an adequate measure of the density evolution in a protoplanetary disk.

\subsection{Particle Response with Increasing Planet} Mass

The particle response to a changing/increasing planet mass is also studied.

Simulations with an initial planet mass $\mu_{i}=10^{-6}$ and a final mass $\mu_{f}=10^{-4}$ showed that particle can be captured into different orbits. The turn-on time was 100P. Fig. 12.7 shows particle paths starting near point $L_{3}$ (to the left) at different $\Delta r$.

While the planet mass increases, some particles are captured from an initial horseshoe orbit into a tadpole orbit. This only happenes if the orbit intersects with the increasing Roche lobe radius before it comes close to the planet. Particles at faster horseshoe orbits pass the planet before they are able to be captured. While the planet mass grows also the regions for horseshoe orbits and tadpole orbits grow. This behavior has already been studied to some extent by Fleming \& Hamilton (2000) for the case of Jupiter The results are not significantly affected by the type of mass growth (exponential or linear).

The Jacoby constant is the only constant in the restricted three-body system. However, that is only true for a constant planet mass. Once the planet changes its 


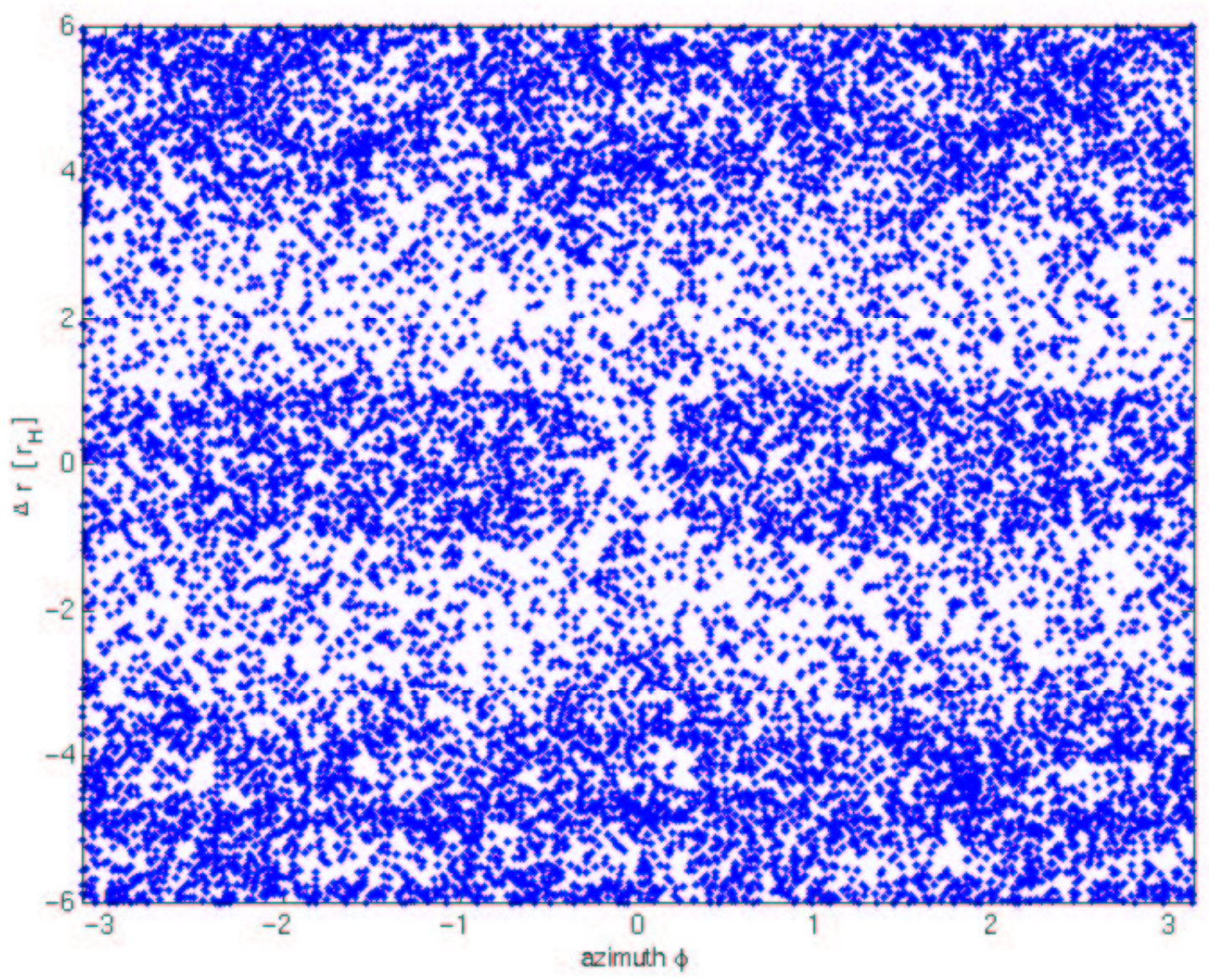

Figure 12.5: Snapshot of the location of one million particles from the restricted three-body code after 300 orbits. The particles were initially randomly distributed over an annulus between $-6 r_{H}<\Delta r<6 r_{H}$ with a density distribution $\propto r^{-3 / 2}$. The planet is located at $(\Delta r, \phi)=(0,0)$. Particles in Region $2\left(r_{H}<\Delta r<\sqrt{12} r_{H}\right)$ are strongly perturbed by the planet and their orbit is distributed over a much larger area. Consequently, the density in this region drops by a factor of 2 whereas other regions (horseshoe and streaming region) are slightly increased in density. 


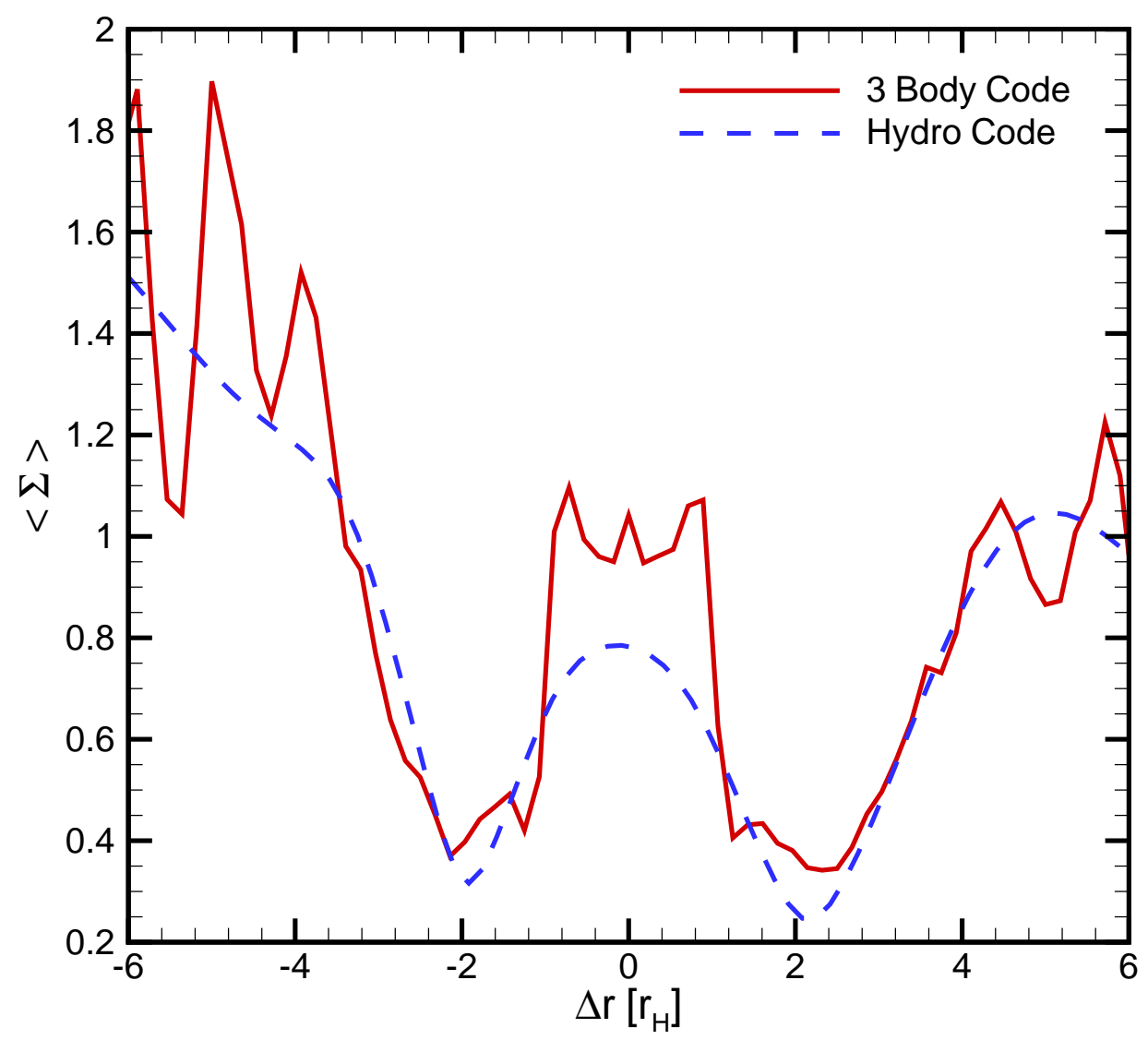

Figure 12.6: Azimuthally averaged particle number density from the restricted three-body code (red solid) compared with the density from a hydrodynamic simulation (blue dashed) after $t=300 P$. The number density has been normalized. The initial density distribution in both simulation is $\propto r^{-3 / 2}$. The profiles from the two codes match well within the density dips. The density in other regions is more enhanced in the particle code than in the hydro code. 
mass the Jacoby constant $C_{j}$ changes too. That means particles can now cross into regions they were previously excluded from. Once the planet mass stops growing, $C_{j}$ will be a constant again.

\subsection{Analytic Approach to Smoothing Factor}

Although the restricted three-body problem in not integrable in an analytic way, we can find special solutions, i.e. libration points. This can be done by searching for points where the particle has zero velocity and acceleration in the rotating frame. The procedure can be looked up in many books, e.g. Murray \& Dermott (1999). These libration points are usually calculated with an exact potential from two point masses. Since the hydro simulations applies a smoothing factor to the potential of the planet to avoid the singularity at that position, also the location of the libration points will be affected.

Let us consider the equations of motion written as

$$
\begin{aligned}
& \ddot{x}-2 \omega \dot{y}=\frac{\partial U}{\partial x} \\
& \ddot{y}+2 \omega \dot{x}=\frac{\partial U}{\partial y}
\end{aligned}
$$

but with, $\ddot{x}=\ddot{y}=\dot{x}=\dot{y}=0$, where $U=U(x, y)$ is given by

$$
U=\frac{\omega^{2}}{2}\left(x^{2}+y^{2}\right)+\frac{\mu_{1}}{r_{1}}+\frac{\mu_{2}}{r_{2}} .
$$




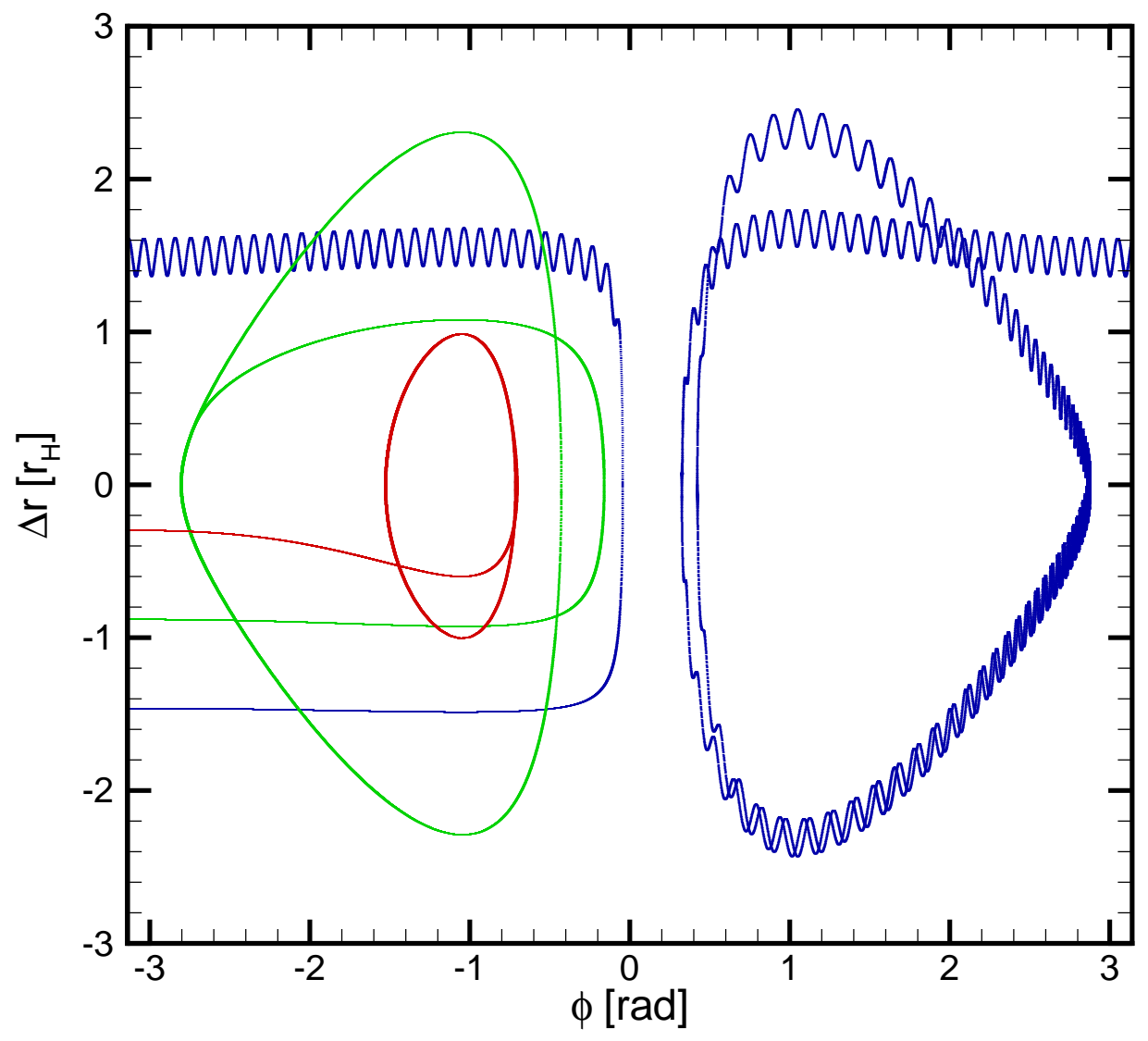

Figure 12.7: Three particle orbits with an increasing planet mass. Starting positions were near $L_{3}$ at $0.3 r_{H}$ (red), $0.87 r_{H}$ (green), $1.47 r_{H}$ (blue). While the planet mass increased, particles are captured from horseshoe orbits into tadpole orbits. The planet is located at $(\Delta r, \phi)=(0,0)$. 
In this equation the term $x^{2}+y^{2}$ is the centrifugal potential and the terms $1 / r_{1,2}$ are the gravitational potential of the star and the planet. Their partial derivative gives rise to centrifugal and gravitational force.

The distance of a particle to the star $r_{1}$ and to the planet $r_{2}$ including a smoothing factor $s$ can be expressed as

$$
\begin{aligned}
& r_{1}^{2}=\left(x+\mu_{2}\right)^{2}+y^{2} \\
& r_{2}^{2}=\left(x-\mu_{1}\right)^{2}+y^{2}+s^{2} .
\end{aligned}
$$

Only the planet receives a smoothing factor. We find the two new solution for $L_{4}$ and $L_{5}$ depending on the smoothing factor following the procedure in Murray \& Dermott (1999)

$$
\begin{aligned}
& x=\frac{1}{2}-\mu_{2}+\frac{s^{2}}{2} \\
& y=\left[\frac{3}{4}-\frac{s^{2}}{2}\left(\frac{s^{2}}{2}+1\right)\right]^{1 / 2} .
\end{aligned}
$$

Therefore, the location of the libration points depends only to second order on the smoothing factor. Since $s$ is to the order of $r_{H}$ and therefore small, the change of particle paths by including a smoothing factor into the potential of the planet is negligible. 


\section{Chapter 13}

\section{Results and Discussion}

High resolution two-dimensional hydrodynamic disk simulation were carried out with one or two embedded protoplanets. The tidal interaction between a protostellar disk and an embedded planet with a modest mass is studied. Linear torque calculations suggest that such interaction leads to angular momentum transfer and inward migration of the planets. For planets with mass comparable to that of Earth, the inferred migration time scale (Ward, 1997) is much shorter than the observationally determined depletion time scale of the disk (Haisch et al., 2001). This analytic result raises a problem for the formation of giant planets through the core accretion process (Pollack et al., 1996). This process was re-investigated with this research in an attempt to resolve this paradox.

The embedded protoplanet induces a circulation flow pattern near its corotation region in the disk. One key result here is that even in disks with an initially 
uniform potential vorticity, inflection points may emerge and grow spontaneously as potential vorticity is generated near the spiral shock in the vicinity of the protoplanet. These inflection points lead to the onset and growth of secondary instabilities, i.e., vortices. The existence of secondary instabilities and their non-linear outcome as vortices indicate that the flows in the co-orbital region are perhaps more complicated than linear analysis have suggested. These vortices should exert strong torques on the planet.

Another key result of this research is that the total torque on the planet, caused by tidal interactions between the disk and the planet, can be divided into three stages: Phase I is negative, smooth, and modulated by a period roughly corresponding the libration period. Phase II shows large amplitudes and very fast oscillations with an approximate period of one orbit. In phase III vortices merge and torques become even larger but the oscillation has a lower frequency. This last transition is only observed for lower sound speed runs $c_{s}=0.2,0.32,0.4$ but presumably will also occur at later times for intermediate sound speed simulations.

It is also discussed that simulations with different sound speeds group into three regimes according to the disk response: $c_{s}<0.04$ (low), $0.04 \leq c_{s} \leq 0.06$ (intermediate), and $c_{s}>0.06$ (high). Intermediate sound speed simulations show a distinct phase I with a transition to phase II and III, whereas in low sound speed runs secondary instabilities develop early on even during the turn-on time for the planet mass. Therefore, the torque evolution does not not show a clear phase I 
behavior but only phase II and III. High sound speed simulation have a very small growth rate in the disk response. The runs do not show a distinct transition from phase I to phase II but it will presumably occur at a later stage.

By investigating simulations with different parameters it can be concluded that the disk is responding to the perturber with a function of planet mass and sound speed. The growth rate for a constant $\mu$ is linear in time and a function of $\mu^{2}$. A clear relation between growth rate and sound speed could not be found but is probably due to the grouping into three different regimes. However, the threshold condition for the intermediate sound speed simulations can be described as $\propto c_{s}^{-0.8}$. Another key finding of this work is that the parameter $K \propto c_{s} / r_{H}$ determines the shock location and consequently the disk response. Therefore, the growth rate of the potential vorticity profile and the threshold condition for developing secondary instabilities are a function of $K$ as well.

The details of the shock structure were also described and why they lead to the development of extrema in the PV profile. The profile depends on the location where the particle moves through the shock but that location is shifted due to the attractive potential of the planet. The structures in the PV profile are a function of several parameters including pre-shock density, tangential gradients of the normal velocity component, and Mach number at the point where the particle crosses through the shock. Is was shown in $\S 9$ that the loss or gain from the shock can be matched to a large degree with a simple analytic expression in regions further 
away from the planet (region III and II). Region I is where the flow lines cross at the tip of the shock and, also, they are heavily influenced by the potential of the planet. It remains uncertain if a simple analytical expression can explain this region or if more physical properties have to be considered. Unfortunately, region I is the area that becomes unstable first by forming vortices. This influences the rest of the disk especially when these vortices grow and merge. Region III and II never became unstable in the simulations except in the multi planet studies.

At this point, the dip in region I is difficult to explain and the physics hard to pin down. The formation of the dip could be very well to a numerical problem but it could be also due to physics that has not been considered for the analytical formula. Region I is very similar to a boundary layer problem with Kelvin-Helmholtz instabilities: An increase in resolution leads to a decreasing depth of the boundary layer. Resolution tests showed that the dip in region I becomes less and less pronounced. The question remains, if it will vanish at a certain resolution.

A comparison with VH1 was not that helpful because of the numerical noise. A different code, like FLASH from the University of Chicago using an adaptive mesh refinement, could solve the problem. Another approach would be to change the shock capturing method in the hybrid code and find a way to rewrite the Euler equation such that potential vorticity is conserved with each time step.

In a private communication, Kley (2004) announced that his code also shows developing vortices. He has done studies with different viscosities and found that 
vortices vanish by using a higher alpha-type viscosity of $>10^{-3}$.

This research also compared the results from hydrodynamic simulation with simple restricted three-body simulations. At the low temperature limit, the density "W" profile matches well with an azimuthally averaged number density of a three body simulation. Since the particles in the separatrix region suffer a strong force from the planet, they are kicked into high eccentricity orbits. These orbits are distributed over a larger radial extent and therefore the number density drops within the separatrix region.

One limitation of the current study is that the planet is being artificially held on a fixed circular orbit. Such strong and rapidly varying torques from vortices might cause the planet to "oscillate" and lose its phase coherence with the surrounding flow. This feedback process should have important implications for the type I migration problem. Allowing the planet to migrate radially must be included for understanding the planet's response to such torques. 


\section{Bibliography}

Balmforth, N. J. \& Korycansky, D. G. 2001, MNRAS, 326, 833

Boss, A. P. 2001a, ApJ, 551, L167

—. 2001b, ApJ, 563, 367

Bryden, G., Chen, X., Lin, D. N. C., Nelson, R. P., \& Papaloizou, J. C. B. 1999, ApJ, 514, 344

Bryden, G., Różyczka, M., Lin, D. N. C., \& Bodenheimer, P. 2000, ApJ, 540, 1091

Colella, P. \& Woodward, P. 1984, J.Comp.Phys., 54, 174

D’Angelo, G., Henning, T., \& Kley, W. 2002, A\&A, 385, 647

D'Angelo, G., Kley, W., \& Henning, T. 2003, ApJ, 586, 540

Dermott, S. F. \& Murray, C. D. 1981, Icarus, 48, 12

Eggenberger, A., Udry, S., \& Mayor, M. 2004, A\&A, 417, 353

Fleming, H. J. \& Hamilton, D. P. 2000, Icarus, 148, 479

Goldreich, P. \& Tremaine, S. 1979, ApJ, 233, 857

—. 1980, ApJ, 241, 425

Haisch, K. E., Lada, E. A., \& Lada, C. J. 2001, ApJ, 553, L153

Hayes, W. D. 1957, J. Fluid Mech., 2, 595

Henon, M. 1981, Nature, 293, 33

Hollenbach, D. J., Yorke, H. W., \& Johnstone, D. 2000, Protostars and Planets IV, 401

Kevlahan, N. K.-R. 1997, J. Fluid Mech., 341, 371 
Kley, W. 1998, A\&A, 338, L37

-. 1999, MNRAS, 303, 696

—. 2004, priv. comm.

Kley, W., D’Angelo, G., \& Henning, T. 2001, ApJ, 547, 457

Koller, J. \& Li, H. 2003, in ASP Conf. Ser. 294: Scientific Frontiers in Research on Extrasolar Planets, 339-342

Kornet, K., Różyczka, M., \& Stepinski, T. F. 2004, A\&A, 417, 151

Kornreich, P. \& Scalo, J. 2000, ApJ, 531, 366

Laney, C. B. 1998, Computational Gasdynamics (Cambridge University Press)

Li, H., Colgate, S. A., Wendroff, B., \& Liska, R. 2001, ApJ, 551, 874

Lighthill, M. J. 1957, J. Fluid Mech., 2, 1

Lin, D. N. C. \& Papaloizou, J. 1985, in Protostars and Planets II, 981-1072

Lin, D. N. C. \& Papaloizou, J. 1986a, ApJ, 307, 395

-. 1986b, ApJ, 309, 846

Lin, D. N. C. \& Papaloizou, J. C. B. 1993, in Protostars and Planets III, 749-835

Lissauer, J. J., Shu, F. H., \& Cuzzi, J. N. 1981, Nature, 292, 707

Lubow, S. H., Seibert, M., \& Artymowicz, P. 1999, ApJ, 526, 1001

Marcy, G. W. \& Butler, R. P. 1998, ARA\&A, 36, 57

Marcy, G. W., Cochran, W. D., \& Mayor, M. 2000, Protostars and Planets IV, 1285

Masset, F. 2000, A\&AS, 141, 165

Masset, F. S. 2001, ApJ, 558, 453

-. 2002, A\&A, 387, 605

Mayer, L., Quinn, T., Wadsley, J., \& Stadel, J. 2002, Science, 298, 1756

Mayor, M. \& Queloz, D. 1995, Nature, 378, 355 
Murray, C. D. \& Dermott, S. F. 1999, Solar system dynamics (Cambridge University Press)

Murray, N., Hansen, B., Holman, M., \& Tremaine, S. 1998, Science, 279, 69

Nelson, R. P., Papaloizou, J. C. B., Masset, F., \& Kley, W. 2000, MNRAS, 318, 18

Papaloizou, J. \& Lin, D. N. C. 1984, ApJ, 285, 818

Petit, J.-M. \& Henon, M. 1988, A\&A, 199, 343

Pollack, J. B., Hubickyj, O., Bodenheimer, P., Lissauer, J. J., Podolak, M., \& Greenzweig, Y. 1996, Icarus, 124, 62

Rasio, F. A. \& Ford, E. B. 1996, Science, 274, 954

Sandquist, E. L., Dokter, J. J., Lin, D. N. C., \& Mardling, R. A. 2002, ApJ, 572, 1012

Santos, N. C., Israelian, G., Mayor, M., Rebolo, R., \& Udry, S. 2003, A\&A, 398, 363

Toro, E. F. 1999, Riemann Solvers and Numerical Methods for Fluid Dynamics (Springer)

Trilling, D. E., Lunine, J. I., \& Benz, W. 2002, A\&A, 394, 241

Truesdell, C. 1952, J. Aero. Sci., 19, 826

Udry, S., Mayor, M., \& Santos, N. C. 2003, A\&A, 407, 369

Ward, W. R. 1997, Icarus, 126, 261

Weidenschilling, S. J. \& Marzari, F. 1996, Nature, 384, 619

Weisstein, E. W. 2004, http://mathworld.wolfram.com/VectorDerivative.html, MathWorld - A Wolfram Web Resource

Wolszczan, A. 1994, Science, 264, 538

Wolszczan, A. \& Frail, D. A. 1992, Nature, 355, 145 

This report has been reproduced directly from the best available copy. It is available electronically on the Web (http://www.doe.gov/bridge).

Copies are available for sale to U.S. Department of Energy employees and contractors from:

Office of Scientific and Technical Information P.O. Box 62

Oak Ridge, TN 37831

(865) 576-8401

Copies are available for sale to the public from: National Technical Information Service

U.S. Department of Commerce

5285 Port Royal Road

Springfield, VA 22161

(800) 553-6847 


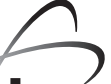

- Los Alamos

NATIONAL LABORATORY 